BULLETIN (New Series) OF THE AMERICAN MATHEMATICAL SOCIETY

Volume 34, Number 4, October 1997, Pages 339-403

S 0273-0979(97)00732-5

\title{
THE SYMMETRIES OF SOLITONS
}

\author{
RICHARD S. PALAIS
}

\begin{abstract}
In this article we will retrace one of the great mathematical adventures of this century - the discovery of the soliton and the gradual explanation of its remarkable properties in terms of hidden symmetries. We will take an historical approach, starting with a famous numerical experiment carried out by Fermi, Pasta, and Ulam on one of the first electronic computers, and with Zabusky and Kruskal's insightful explanation of the surprising results of that experiment (and of a follow-up experiment of their own) in terms of a new concept they called "solitons". Solitons however raised even more questions than they answered. In particular, the evolution equations that govern solitons were found to be Hamiltonian and have infinitely many conserved quantities, pointing to the existence of many non-obvious symmetries. We will cover next the elegant approach to solitons in terms of the Inverse Scattering Transform and Lax Pairs, and finally explain how those ideas led step-by-step to the discovery that Loop Groups, acting by "Dressing Transformations", give a conceptually satisfying explanation of the secret soliton symmetries.
\end{abstract}

\section{Contents}

1. Introduction 340

2. Review of Classical Mechanics 342

1. Newton's Equations 342

2. The Lagrangian Viewpoint 343

3. Noether's Principle 344

4. The Hamiltonian Viewpoint 345

5. Symplectic Manifolds 346

6. Examples of Classical Mechanical Systems 349

7. Physics Near Equilibrium 351

8. Ergodicity and Thermalization 353

3. Origins of Soliton Theory 355

1. The Fermi-Pasta-Ulam Experiments 355

2. The Kruskal-Zabusky Experiments 359

3. A First Look at KdV 361

4. "Steepening" and "Breaking" 363

5. Dispersion 365

6. Split-Stepping KdV 365

Received by the editors May 7, 1997, and in revised form, August 6, 1997.

1991 Mathematics Subject Classification. Primary 58F07, 35Q51, 35Q53, and 35Q55.

Key words and phrases. Solitons, integrable systems, hidden symmetry, Korteweg-de Vries equation, Nonlinear Schrödinger equation, Lax pair, Inverse Scattering Transform, loop group.

During the preparation of this paper, the author was supported in part by the Mathematics Institute and Sonderforschungsbereich 256 of Bonn University. 
7. A Symplectic Structure for KdV 366

4. The Inverse Scattering Method 369

1. Lax Equations: KdV as an Isospectral Flow 370

2. The Scattering Data and Its Evolution 373

3. The Inverse Scattering Transform 375

4. An Explicit Formula for KdV Multi-Solitons 376

5. The KdV Hierarchy 377

5. The ZS-AKNS Scheme 379

1. Flat Connections and the Lax Equation, ZCC 379

2. Some ZS-AKNS Examples 380

3. The Uses of Solitons 381

4. Nonlinear Schrödinger as a Hamiltonian Flow 384

5. The Nonlinear Schrödinger Hierarchy 386

6. ZS-AKNS Direct Scattering Theory 389

1. Statements of Results 389

2. Outline of Proofs 391

7. Loop Groups, Dressing Actions, and Inverse Scattering 395

1. Secret Sources of Soliton Symmetries 395

2. Terng-Uhlenbeck Factoring and the Dressing Action 396

3. The Inverse Scattering Transform 397

4. ZS-AKNS Scattering Coordinates 398

References $\quad 400$

\section{INTRODUCTION}

In the past several decades, two major themes have dominated developments in the theory of dynamical systems. On the one hand there has been a remarkable and rapid development in the theory of so-called "chaotic" systems, with a gradual clarification of the nature and origins of the surprising properties from which these systems get their name. Here what cries out to be explained is how a system that is deterministic can nevertheless exhibit behavior that appears erratic and unpredictable.

In this article I will be discussing a second class of systems - equally puzzling, but for almost the opposite reason. For these so-called "integrable systems", the challenge is to explain the striking predictability, regularities, and quasi-periodicities exhibited by their solutions, a behavior particularly apparent for a special class of solutions, called "solitons". The latter exhibit a "particle-like" behavior that gives them their name; for example they have geometric shapes that show a remarkable degree of survivability under conditions that one might normally expect to destroy such features.

Such conservation of geometric features is known to be intimately bound up with notions of symmetry - in fact, when suitably formalized, a famous theorem of E. Noether states that conserved quantities correspond to one-parameter groups of automorphisms of the dynamical system - and therein lies a puzzle. These systems do not have manifestly obvious symmetries to account for these anomalous conservation laws, and to fully understand their surprising behavior we must search for the secret sources of their hidden symmetries. This article will be about that search and about the many mathematical treasures it has so far revealed. 
A major problem for anyone attempting an exposition of "soliton mathematics" or "integrable systems" is the vast extent of its literature. The theory had its origins in the 1960 's, and so can be considered relatively recent. But early research in the subject revealed mysterious new mathematical phenomena that quickly attracted the attention and stimulated the curiosity of many mathematicians throughout the world. As these researchers took up the intriguing challenge of understanding these new phenomena, an initial trickle of papers soon grew to a torrent, and the eventual working out of the details of the theory resulted from a concerted effort by hundreds of mathematicians whose results are spread over a still growing bibliography of many thousands of papers.

Attempting to cover the subject in sufficient detail to mention all these contributions - or even most of the important contributions - would require hundreds of pages. I have neither the time nor the expertise to undertake such a task, and instead I have tried to provide a guided tour through what I consider some of the major highlights of the subject. But the reader should realize that any attempt to compress such a massive subject in so few pages must be an exercise in selectivity that will in large measure reflect personal taste and biases of the author rather than some objective measure of importance.

Another disclaimer: as we proceed I will try to present some of the remarkable story of how the subject began and developed. I say "story" rather than "history" because my report will be anecdotal in nature. I will try to be accurate, but I do not pretend to have done careful historical research. It is particularly important to keep in mind that during most of the development of the theory of integrable systems there was a very large and active group of mathematicians working on the subject in the former Soviet Union. Since communication of results between this group and the group of western mathematicians working in the field was slower than that within each group, even more than usual there were frequent cases in which similar advances were made nearly simultaneously in one group and the other. Statements made in this article to the effect that some person discovered a certain fact should not be interpreted as claiming that person had priority or sole priority in the discovery.

There have been a number of fine volumes written that make a serious effort to encompass the bulk of soliton theory, giving careful historical and bibliographic references. I hope my abbreviated account will stimulate readers to consult these more complete sources, several of which are listed in the references $([\mathrm{AC}],[\mathrm{FT}],[\mathrm{N}]$, [NMPZ]).

The organization of this article will be in part historical. We will start with some surprising numerical experiments of Fermi-Pasta-Ulam and of Zabusky-Kruskal that were the origins of soliton theory. We will next consider the remarkable Inverse Scattering Transform and the related concept of Lax Pairs, first in the original context of the Korteweg-de Vries (KdV) equation, and then for the more general hierarchies of integrable systems introduced by Zakharov and Shabat and by Ablowitz, Kaup, Newell, and Segur (ZS-AKNS). We will trace how developments that grew out of the ZS-AKNS approach eventually led to a synthesis that explains most of the phenomena of soliton theory from a unified viewpoint. In particular, it uncovers the source of the hidden symmetries of solitons, explaining both the existence of so many commuting constants of the motion and also the characteristic phenomenon of Bäcklund Transformations. This synthesis had its origins in the idea of "dressing transformations", and in explaining it I will follow the recent approach of Chuu-lian 
Terng and Karen Uhlenbeck. I would like to express my sincere thanks to Chuu-lian for putting up with my countless requests that she interrupt her own work in order to explain to me some detail of this approach. Without these many hours of help, it would not have been possible for me to complete this article.

This article is a revised version of notes from a series of Rudolf-Lipschitz Lectures that I delivered at Bonn University in January and February of 1997. I would like to thank the Mathematisches Institut of Universität Bonn and its Sonderforschungsbereich 256 for honoring me with the invitation to give that lecture series, and to thank the lively and high-level audience who, by their interest, stimulated me to write up my rough notes.

My thanks to Bob Palais for pointing out a problem in my original discussion of split-stepping - and for helping me to re-write it.

And special thanks to Barbara Beeton for an exceptional job of proof-reading. The many changes she suggested have substantially improved readability.

\section{Review of Classical Mechanics}

In this section we will review Classical Mechanics, in both the Lagrangian and Hamiltonian formulations. This is intended mainly to establish notational conventions, not as an exposition for novices. We shall also review the basic geometry of symplectic manifolds.

1. Newton's Equations. Let $\mathcal{C}$ be a Riemannian manifold ("configuration space") and $\Pi: T \mathcal{C} \rightarrow \mathcal{C}$ its tangent bundle. A vector field $X$ on $T \mathcal{C}$ is called a second order ODE on $\mathcal{C}$ if $D \Pi\left(X_{v}\right)=v$ for all $v$ in $T \mathcal{C}$. If $\gamma$ is a solution curve of $X$ and $\sigma=\Pi(\gamma)$ is its projection onto $\mathcal{C}$, then, by the chain rule, $\sigma^{\prime}(t)=D \Pi\left(\gamma^{\prime}(t)\right)=$ $D \Pi\left(X_{\gamma(t)}\right)=\gamma(t)$, i.e., $\gamma$ is the velocity field of its projection. An easy argument shows conversely that if this is true for all solutions of a vector field $X$ on $T \mathcal{C}$, then $X$ is a second order ODE on $\mathcal{C}$. For this reason we shall say that a smooth curve $\sigma(t)$ in $\mathcal{C}$ satisfies the second order ODE $X$ if $\sigma^{\prime}$ is a solution curve of $X$.

Given coordinates $x_{1}, \ldots, x_{n}$ for $\mathcal{C}$ in $O$, we define associated "canonical" coordinates $q_{1}, \ldots, q_{n}, \dot{q}_{1}, \ldots, \dot{q}_{n}$ in $\Pi^{-1}(O)$ by $q_{i}=x_{i} \circ \Pi$ and $\dot{q}_{i}=d x_{i}$. Let $\sigma:[a, b] \rightarrow \mathcal{C}$ be a smooth curve in $\mathcal{C}, \sigma^{\prime}:[a, b] \rightarrow T \mathcal{C}$ its velocity. If we define $x_{i}(t)=x_{i}(\sigma(t))$ and $q_{i}(t)=q_{i}\left(\sigma^{\prime}(t)\right)=x_{i}(t)$, then $\dot{q}_{i}(t):=\dot{q}_{i}\left(\sigma^{\prime}(t)\right)=d x_{i}\left(\sigma^{\prime}(t)\right)=\frac{d x_{i}(t)}{d t}=\frac{d q_{i}(t)}{d t}$. It follows that a vector field $X$ on $\mathcal{C}$ is a second order ODE if and only if in each canonical coordinate system it has the form $X=\sum_{i}\left(\dot{q}_{i} \partial / \partial q_{i}+F\left(q_{i}, \dot{q}_{i}\right) \partial / \partial \dot{q}_{i}\right)$, or equivalently the condition for $\sigma^{\prime}$ to be a solution of $X$ is that $d q_{i}(t) / d t=\dot{q}_{i}(t)$, $d \dot{q}_{i}(t) / d t=F_{i}\left(q_{i}(t), \dot{q}_{i}(t)\right)$ (so $d^{2} x_{i}(t) / d t^{2}=F_{i}\left(x_{i}(t), d x_{i}(t) / d t\right)$, explaining why it is called a second order ODE).

The classic example of a second order ODE on $\mathcal{C}$ is the vector field $X$ generating the geodesic flow on $T \mathcal{C}$ - for each $v$ in $T \mathcal{C}$ the solution curve of $X$ with initial condition $v$ is $\sigma^{\prime}$ where $\sigma(t)=\exp (t v)$ is the unique geodesic on $\mathcal{C}$ with $\sigma^{\prime}(0)=v$. In local coordinates, $x_{i}(\sigma(t))$ satisfy the system:

$$
\frac{d^{2} x_{i}}{d t^{2}}=-\Gamma_{j k}^{i}(x) \frac{d x_{j}}{d t} \frac{d x_{k}}{d t}
$$

(where the $\Gamma_{j k}^{i}$ are the Christoffel symbols). What we shall call Newton's Equations (NE) for $\mathcal{C}$ is a second order ODE $X^{U}$ for $\mathcal{C}$ that is a slight generalization of the geodesic flow and is determined by a smooth real-valued function $U$ on $\mathcal{C}$ called the 
potential energy function:

$$
\frac{d^{2} x_{i}}{d t^{2}}=-\Gamma_{j k}^{i}(x) \frac{d x_{j}}{d t} \frac{d x_{k}}{d t}-\frac{\partial U}{\partial x_{i}} .
$$

[Here is an intrinsic, geometric description of (NE). The gradient of $U, \nabla U$ is a vector field on $\mathcal{C}$, and we call $-\nabla U$ the force. If $\sigma(t)$ is any smooth curve in $\mathcal{C}$, and $v(t)$ is any tangent vector field along $\sigma$ (i.e., a lifting of $\sigma$ to $T \mathcal{C}$ ), then the Levi-Civita connection allows us to covariantly differentiate $v$ along $\sigma$ to produce another vector field $D v / d t$ along $\sigma$. In particular, if for $v$ we take the velocity field $\sigma^{\prime}(t)$, we can interpret $D \sigma^{\prime} / d t$ as the acceleration of $\sigma$, and the curve $\sigma$ satisfies Newton's Equations (for the potential $U$ ) if and only if $D \sigma^{\prime} / d t=-\nabla U$.]

2. The Lagrangian Viewpoint. We define the kinetic energy function $K$ on $T \mathcal{C}$ by $K(v)=\frac{1}{2}\|v\|^{2}$, and we also consider the potential energy as a function on $T \mathcal{C}$ by $U(v)=U(\Pi(v))$. Their difference $\mathcal{L}=K-U$ is called the Lagrangian function on $T \mathcal{C}$, and if $\sigma:[a, b] \rightarrow \mathcal{C}$ is any smooth curve in $\mathcal{C}$, we define its action $A(\sigma)=$ $\int_{a}^{b} \mathcal{L}\left(\sigma^{\prime}(t)\right) d t$. In canonical coordinates as above, $\mathcal{L}(q, \dot{q})=\frac{1}{2} \sum_{i j} g_{i j} \dot{q}_{i} \dot{q}_{j}-U(q)$, so if we write $x_{i}(t)=x_{i}(\sigma(t))$, then $q_{i}\left(\sigma^{\prime}(t)\right)=x_{i}(t), \dot{q}_{i}\left(\sigma^{\prime}(t)\right)=d x_{i} / d t$, and therefore

$$
A(\sigma)=\int_{a}^{b} \mathcal{L}(q(t), \dot{q}(t)) d t=\int_{a}^{b} \frac{1}{2} \sum_{i j} g_{i j}(x(t)) \frac{d x_{i}}{d t} \frac{d x_{j}}{d t}-U(x(t)) d t .
$$

Let $\sigma_{\epsilon}:[a, b] \rightarrow \mathcal{C}$ be a smooth one-parameter family of curves defined for $\epsilon$ near zero, and with $\sigma_{0}=\sigma$. If we define $\delta \sigma=\left(\frac{d}{d \epsilon}\right)_{\epsilon=0} \sigma_{\epsilon}$ (a vector field along $\sigma)$, then it is easy to see that $\left(\frac{d}{d \epsilon}\right)_{\epsilon=0} A\left(\sigma_{\epsilon}\right)$ depends only on $\sigma$ and $\delta \sigma$, and we denote it by $D A_{\sigma}(\delta \sigma)$. Define $q_{i}(t, \epsilon)=q_{i}\left(\sigma_{\epsilon}^{\prime}(t)\right)=x_{i}\left(\sigma_{\epsilon}(t)\right), \delta q_{i}(t)=\partial q_{i}(t, 0) / \partial \epsilon$, $\dot{q}_{i}(t, \epsilon)=\dot{q}_{i}\left(\sigma_{\epsilon}^{\prime}(t)\right)$ and $\delta \dot{q}_{i}(t)=\partial \dot{q}_{i}(t, 0) / \partial \epsilon$. Then clearly $\dot{q}_{i}(t, \epsilon)=\partial q_{i}(t, \epsilon) / \partial t$, so, by equality of cross derivatives, $\delta \dot{q}_{i}(t)=\frac{d}{d t} \delta q_{i}$.

It is now easy to compute $D A_{\sigma}(\delta \sigma)$. In fact, differentiating under the integral sign, using the chain rule, and integrating by parts give:

$$
\begin{aligned}
D A_{\sigma}(\delta \sigma) & =\int_{a}^{b} \sum_{i}\left(\frac{\partial \mathcal{L}}{\partial q_{i}} \delta q_{i}+\frac{\partial \mathcal{L}}{\partial \dot{q}_{i}} \delta \dot{q}_{i}\right) d t \\
& =\int_{a}^{b} \sum_{i}\left(\frac{\partial \mathcal{L}}{\partial q_{i}}-\frac{d}{d t} \frac{\partial \mathcal{L}}{\partial \dot{q}_{i}}\right) \delta q_{i} d t+\left[\sum_{i} \frac{\partial \mathcal{L}}{\partial \dot{q}_{i}} \delta q_{i}\right]_{a}^{b} \\
& =\int_{a}^{b} \sum_{i}\left(\frac{\partial \mathcal{L}}{\partial q_{i}}-\frac{d}{d t} \frac{\partial \mathcal{L}}{\partial \dot{q}_{i}}\right) \delta q_{i} d t+\left[\left\langle\sigma^{\prime}(t), \delta \sigma(t)\right\rangle\right]_{a}^{b} .
\end{aligned}
$$

The curve $\sigma$ is called a critical point of the action functional $A$ if $D A_{\sigma}(\delta \sigma)$ vanishes for all variations $\delta \sigma$ vanishing at the endpoints $a$ and $b$, or equivalently if the Euler-Lagrange equations $\frac{\partial \mathcal{L}}{\partial q_{i}}-\frac{d}{d t} \frac{\partial \mathcal{L}}{\partial \dot{q}_{i}}=0$ are satisfied. Substituting in the expression for $\mathcal{L}(q, \dot{q})$ above, and recalling the definition of the Christoffel symbols, one can easily check that $\sigma$ is a critical point of the action functional if and only if it satisfies Newton's Equations.

It follows that if $\sigma$ is a solution of Newton's Equations, then for any variation $\delta \sigma$, not necessarily vanishing at the endpoints,

$$
D A_{\sigma}(\delta \sigma)=\left[\left\langle\sigma^{\prime}(t), \delta \sigma(t)\right\rangle\right]_{a}^{b} .
$$


As a first application, consider the variation of $\sigma$ defined by $\sigma_{\epsilon}(t)=\sigma(t+\epsilon)$. Clearly $\delta \sigma(t)=\sigma^{\prime}(t)$ and $A\left(\sigma_{\epsilon}\right)=\int_{a+\epsilon}^{b+\epsilon} \mathcal{L}\left(\sigma^{\prime}\right) d t$, so the definition of $D A_{\sigma}(\delta \sigma)$ gives $D A_{\sigma}(\delta \sigma)=\left[\mathcal{L}\left(\sigma^{\prime}(t)\right)\right]_{a}^{b}$, while the above general formula for $D A_{\sigma}(\delta \sigma)$ when $\sigma$ satisfies (NE) gives $D A_{\sigma}(\delta \sigma)=\left[\left\|\sigma^{\prime}(t)\right\|^{2}\right]_{a}^{b}=\left[2 K\left(\sigma^{\prime}(t)\right)\right]_{a}^{b}$.

If we define the Hamiltonian or total energy function $H$ on $T C$ by $H=2 K-\mathcal{L}=$ $2 K-(K-U)=K+U$, then it follows that $\left[H\left(\sigma^{\prime}\right)\right]_{a}^{b}=0$, or in other words $H$ is constant along $\sigma^{\prime}$ whenever $\sigma$ is a solution of Newton's Equations. Now a function $F$ on $T \mathcal{C}$ that is constant along $\sigma^{\prime}$ whenever $\sigma:[a, b] \rightarrow \mathcal{C}$ satisfies (NE) is called a constant of the motion for Newton's Equations, so we have proved:

Conservation of Energy Theorem. The Hamiltonian $H=K+U$ is a constant of the motion for Newton's Equations.

[Here is a more direct proof. $K\left(\sigma^{\prime}\right)=\frac{1}{2} g\left(\sigma^{\prime}, \sigma^{\prime}\right)$, where $g$ is the metric tensor. By definition of the Levi-Civita connection, $D g / d t=0$, and (NE) says $D \sigma^{\prime} / d t=$ $-\nabla U$, so $d K\left(\sigma^{\prime}\right) / d t=g\left(-\nabla U, \sigma^{\prime}\right)=-d U / d t$.]

3. Noether's Principle. A diffeomorphism $\phi$ of $\mathcal{C}$ induces a diffeomorphism $D \phi$ of $T \mathcal{C}$, and we call $\phi$ a symmetry of Newton's Equations if $D \phi$ preserves $\mathcal{L}$, i.e., if $\mathcal{L} \circ D \phi=\mathcal{L}$. In particular, any isometry of $\mathcal{C}$ that preserves $U$ is a symmetry of (NE). We note that if $\phi$ is a symmetry of (NE) and $\sigma$ is any smooth path in $\mathcal{C}$, then $A(\phi \circ \sigma)=A(\sigma)$, and it follows that $\phi$ permutes the critical points of $A$. Thus if $\sigma$ is a solution of (NE) then so is $\phi \circ \sigma$. A vector field $Y$ is called an infinitesimal symmetry of Newton's equations if it generates a one-parameter group of symmetries of Newton's equations, so in particular any Killing vector field that is tangent to the level surfaces of $U$ is an infinitesimal symmetry of Newton's Equations.

Suppose that $Y$ is any vector field on $\mathcal{C}$ generating a one-parameter group of diffeomorphisms $\phi_{t}$ of $\mathcal{C}$. We associate to $Y$ a function $\hat{Y}$ on $T \mathcal{C}$, called its conjugate momentum function, by $\hat{Y}(v)=\left\langle v, Y_{\Pi(v)}\right\rangle$. If $\sigma$ is any smooth path in $\mathcal{C}$, then we can generate a variation of $\sigma$ defined by $\sigma_{\epsilon}(t)=\phi_{\epsilon}(\sigma(t))$. Then by definition, $\delta \sigma(t)=$ $Y_{\sigma(t)}$; so, by the above general formula, if $\sigma$ is a solution of Newton's Equations, then $D A_{\sigma}(\delta \sigma)=\left[\hat{Y}\left(\sigma^{\prime}(t)\right)\right]_{a}^{b}$. Now suppose $Y$ is an infinitesimal symmetry of Newton's Equations. Then since $A\left(\sigma_{\epsilon}\right)=A\left(\phi_{\epsilon} \circ \sigma\right)=A(\sigma), D A_{\sigma}(\delta \sigma)$ is zero by definition; hence $\left[\hat{Y}\left(\sigma^{\prime}(t)\right)\right]_{a}^{b}=0$, i.e., $\hat{Y}$ is constant along $\sigma^{\prime}$. This proves:

E. Noether's Principle. The conjugate momentum of an infinitesimal symmetry is a constant of the motion.

The conjugate momentum to the vector field $\partial / \partial q_{i}$ is denoted by $P_{i} ; P_{i}=$ $\sum_{j} g_{i j} \dot{q}_{j}=\frac{\partial \mathcal{L}}{\partial \dot{q}_{i}}$, and it follows from the non-degeneracy of the inner-product that we can use $q_{1}, \ldots, q_{n}, P_{1}, \ldots, P_{n}$ as coordinates in $\Pi^{-1}(O)$. The fact that Newton's Equations are equivalent to the Euler-Lagrange equations says that in these coordinates Newton's Equations take the form: $\frac{d q_{i}}{d t}=\dot{q}_{i}$, $\frac{d P_{i}}{d t}=\frac{\partial \mathcal{L}}{\partial q_{i}}$ (i.e, $X^{U}=$ $\left.\sum_{i}\left(\dot{q}_{i} \frac{\partial}{\partial q_{i}}+\frac{\partial \mathcal{L}}{\partial q_{i}} \frac{\partial}{\partial P_{i}}\right)\right)$. Since $\sum_{i} P_{i} \dot{q}_{i}=2 K, H=\sum_{i} P_{i} \dot{q}_{i}-\mathcal{L}$, so $d H=\sum_{i}\left(\dot{q}_{i} d P_{i}+\right.$ $\left.P_{i} d \dot{q}_{i}-\frac{\partial \mathcal{L}}{\partial q_{i}} d q_{i}-\frac{\partial \mathcal{L}}{\partial \dot{q}_{i}} d \dot{q}_{i}\right)=\dot{q}_{i} d P_{i}-\frac{\partial \mathcal{L}}{\partial q_{i}} d q_{i}$, or in other words, $\frac{\partial H}{\partial q_{i}}=-\frac{\partial \mathcal{L}}{\partial q_{i}}$ and $\frac{\partial H}{\partial P_{i}}=\dot{q}_{i}$. Thus Newton's Equations take the very simple and symmetric form (called Hamilton's Equations) $\frac{d q_{i}}{d t}=\frac{\partial H}{\partial P_{i}}, \frac{d P_{i}}{d t}=-\frac{\partial H}{\partial q_{i}}$. Equivalently, the vector field $X^{U}$ has the form $X^{U}=\sum_{i}\left(\frac{\partial H}{\partial P_{i}} \frac{\partial}{\partial q_{i}}-\frac{\partial H}{\partial q_{i}} \frac{\partial}{\partial P_{i}}\right)$. 
4. The Hamiltonian Viewpoint. So far we have looked at the dynamics of Newton's Equations on the tangent bundle $T \mathcal{C}$ of the configuration space. We will refer to this as the Lagrangian viewpoint. Since $\mathcal{C}$ is Riemannian, there is a canonical bundle isomorphism $L: T \mathcal{C} \rightarrow T^{*} \mathcal{C}$ of $T \mathcal{C}$ with the cotangent bundle, which in this setting is called the Legendre transformation. Explicitly, $L(v)(u)=\langle u, v\rangle$. The Hamiltonian viewpoint towards particle mechanics consists in moving the dynamics over to $T^{*} \mathcal{C}$ via the Legendre transformation. Remarkably, the transferred dynamics preserves the natural symplectic structure on $T^{*} \mathcal{C}$, and this fact is the basis for powerful tools for better analyzing the situation. The functions $\mathcal{L} \circ L^{-1}$ and $H \circ L^{-1}$ are still called the Lagrangian and Hamiltonian function respectively and will still be denoted by $\mathcal{L}$ and $H$. By further such abuse of notation we will denote the vector field $D L\left(X^{U}\right)$ on $T^{*} \mathcal{C}$ by $X^{U}$.

Just as with the tangent bundle, coordinates $x_{1}, \ldots, x_{n}$ for $\mathcal{C}$ in $O$ define natural coordinates $q_{1}, \ldots, q_{n}, p_{1}, \ldots, p_{n}$ for the cotangent bundle in $\Pi^{-1} O$. Namely, $q_{i}=$ $x_{i} \circ \Pi$ as before, while the $p_{i}$ are defined by $p_{i}(\ell)=\ell\left(\partial / \partial x_{i}\right)$. It is immediate from the definitions that $q_{i} \circ L=q_{i}$ while $p_{i} \circ L=P_{i}$, so it follows from the calculation above that the vector field $X^{U}$ (i.e., $D L\left(X^{U}\right)$ ) on $T^{*} \mathcal{C}$ describing the dynamics of Newton's Equations is $X^{U}=\sum_{i}\left(\frac{\partial H}{\partial p_{i}} \frac{\partial}{\partial q_{i}}-\frac{\partial H}{\partial q_{i}} \frac{\partial}{\partial p_{i}}\right)$.

There is a natural 1-form $\omega$ on $T^{*} \mathcal{C}$; namely if $\ell$ is a cotangent vector of $\mathcal{C}$, then $\omega_{\ell}=D \Pi^{*}(\ell)$, or in other words, for $Y$ a tangent vector to $T^{*} C$ at $\ell, \omega_{\ell}(Y)=$ $\ell\left(D \Pi(Y)\right.$ ), where $\Pi: T^{*} \mathcal{C} \rightarrow \mathcal{C}$ is the bundle projection. (We note that $\omega$ does not involve the Riemannian metric, and in fact is natural in the sense that if $\phi$ is any diffeomorphism of $\mathcal{C}$ and $\Phi=(D \phi)^{*}$ is the induced diffeomorphism of $T^{*} \mathcal{C}$, then $\Phi^{*}(\omega)=\omega$.) We define the natural 2 -form $\Omega$ on $T^{*} \mathcal{C}$ by $\Omega=d \omega$, so $\Omega$ is exact and hence closed, i.e., $d \Omega=0$.

It is then easy to check that $\omega=\sum_{i} p_{i} d q_{i}$ and hence $\Omega=\sum_{i} d p_{i} \wedge d q_{i}$. An immediate consequence of this is that $\Omega$ is non-degenerate, i.e., the map $v \mapsto i_{v} \Omega$ is an isomorphism of the tangent bundle of $T^{*} \mathcal{C}$ with its cotangent bundle. (Here $i_{v} \Omega(u)=\Omega(v, u)$.) In fact, if $v=\sum_{i}\left(A_{i} \frac{\partial}{\partial q_{i}}+B_{i} \frac{\partial}{\partial p_{i}}\right)$, then $i_{v} \Omega=\sum_{i}\left(A_{i} d p_{i}-B_{i} d q_{i}\right)$. In particular $i_{X^{U}} \Omega=\sum_{i}\left(\frac{\partial H}{\partial p_{i}} d p_{i}+\frac{\partial H}{\partial q_{i}} d q_{i}\right)=d H$.

Any coordinates $q_{1}, \ldots, q_{n}, p_{1}, \ldots, p_{n}$ for $T^{*} \mathcal{C}$ are called "canonical coordinates" provided $\Omega=\sum_{i} d p_{i} \wedge d q_{i}$. It follows that the "equations of motion" for solutions of Newton's Equations take the Hamiltonian form: $\frac{d p_{i}}{d t}=-\frac{\partial H}{\partial q_{i}}, \frac{d q_{i}}{d t}=\frac{\partial H}{\partial p_{i}}$, for any such coordinates. If $H$ happens not to involve a particular $q_{i}$ explicitly, i.e., if $H$ is invariant under the one-parameter group of translations $q_{i} \mapsto q_{i}+\epsilon$, then this $q_{i}$ is called a cyclic variable, and its "conjugate momentum" $p_{i}$ is clearly a constant of the motion since $\frac{d p_{i}}{d t}=-\frac{\partial H}{\partial q_{i}}=0$. If we can find canonical coordinates $q_{1}, \ldots, q_{n}, p_{1}, \ldots, p_{n}$ such that all of the $q_{i}$ are cyclic, then we call these variables action-angle variables, and when such coordinates exist we say that the Hamiltonian system is completely integrable. The solutions of a completely integrable system are very easy to describe in action-angle variables. Note that we have $H=H\left(p_{1}, \ldots, p_{n}\right)$. For each $c$ in $\mathbf{R}^{n}$ we have a submanifold $\Sigma_{c}=\left\{\ell \in T^{*} \mathcal{C} \mid p_{i}(\ell)=c_{i}\right\}$, and since the $p_{i}$ are all constants of the motion, these are invariant submanifolds of the flow. Moreover these submanifolds foliate $T^{*} \mathcal{C}$, and on each of them $q_{1}, \ldots, q_{n}$ are local coordinates. If we define $\omega_{i}(c)=\frac{\partial H}{\partial p_{i}}(c)$, then on $\Sigma_{c}$ Hamilton's Equations reduce to $\frac{d q_{i}}{d t}=\omega_{i}(c)$, so on $\Sigma_{c}$ the coordinates $q_{i}(t)$ of a solution curve are given by $q_{i}(t)=q_{i}(0)+\omega_{i}(c) t$. Frequently the surfaces $\Sigma_{c}$ are compact, in which case it is easy to show that each connected component 
must be an $n$-dimensional torus. Moreover in practice we can usually determine the $q_{i}$ to be the angular coordinates for the $n$ circles whose product defines the torus structure - which helps explain the terminology action-angle variables.

Later we will look in more detail at the problem of determining whether a Hamiltonian system is completely integrable.

5. Symplectic Manifolds. The cotangent bundle of a manifold is the model for what is called a symplectic manifold. Namely, a symplectic manifold is a smooth manifold $P$ together with a closed non-degenerate 2 -form $\Omega$ on $P$. If $F: P \rightarrow \mathbf{R}$ is a smooth real-valued function on $P$, then there is a uniquely determined vector field $X$ on $P$ such that $i_{X} \Omega=d F$, and we call $X$ the symplectic gradient of $F$ and denote it by $\nabla_{s} F$. Thus we can state our observation above by saying that the vector field $X^{U}$ on $T^{*} \mathcal{C}$ is the symplectic gradient of the Hamiltonian function: $X^{U}=\nabla_{s} H$.

By an important theorem of Darboux, ([Ar], Chapter 8) in the neighborhood of any point of $P$ there exist "canonical coordinates" $q_{1}, \ldots, q_{n}, p_{1}, \ldots, p_{n}$ in which $\Omega$ has the form $\sum_{i} d p_{i} \wedge d q_{i}$, and in these coordinates $\nabla_{s} H=\sum_{i}\left(\frac{\partial H}{\partial p_{i}} \frac{\partial}{\partial q_{i}}-\frac{\partial H}{\partial q_{i}} \frac{\partial}{\partial p_{i}}\right)$, or equivalently the solution curves of $\nabla_{s} H$ satisfy Hamilton's equations $\frac{d p_{i}}{d t}=-\frac{\partial H}{\partial q_{i}}$, $\frac{d q_{i}}{d t}=\frac{\partial H}{\partial p_{i}}$.

Before considering Poisson brackets on symplectic manifolds, we first make a short digression to review Lie derivatives. Recall that if $X$ is a smooth vector field on a smooth manifold $M$, generating a flow $\phi_{t}$, and if $T$ is any smooth tensor field on $M$, then the Lie derivative of $T$ with respect to $X$ is the tensor field $\mathcal{L}_{X} T=\left.\frac{d}{d t}\right|_{t=0} \phi_{t}^{*}(T)$. If $\mathcal{L}_{X} T=0$, then we shall say that " $X$ preserves $T$ ", for this is the necessary and sufficient condition that the flow $\phi_{t}$ preserve $T$, i.e., that $\phi_{t}^{*}(T)=T$ for all $t$. There is a famous formula of Cartan for the Lie derivative operator $\mathcal{L}_{X}$ restricted to differential forms, identifying it with the anti-commutator of the exterior derivative operator $d$ and the interior product operator $i_{X}$ :

$$
\mathcal{L}_{X}=d i_{X}+i_{X} d .
$$

If $\theta$ is a closed $p$-form, this gives $\mathcal{L}_{X} \theta=d\left(i_{X} \theta\right)$, so $X$ preserves $\theta$ if and only if the $(p-1)$-form $i_{X} \theta$ is closed. In particular this demonstrates the important fact that a vector field $X$ on a symplectic manifold $P$ is symplectic (i.e., preserves the symplectic form, $\Omega$ ) if and only if $i_{X} \Omega$ is a closed 1 -form (and hence, at least locally, the differential of a smooth function). The well known identity $\mathcal{L}_{[X, Y]}=\left[\mathcal{L}_{X}, \mathcal{L}_{Y}\right]$ implies that the space of symplectic vector fields on $P$ is a Lie algebra, which we can think of as the Lie algebra of the group of symplectic diffeomorphisms of $P$. It is an interesting and useful fact that the space of Hamiltonian vector fields on $P$, i.e., those for which $i_{X} \Omega$ is an exact form, $d F$, is not only a linear subspace, but is even a Lie subalgebra of the symplectic vector fields, and moreover the commutator subalgebra of the symplectic vector fields is included in the Hamiltonian vector fields. To demonstrate this we shall show that if $i_{X} \Omega$ and $i_{Y} \Omega$ are closed forms, then $i_{[X, Y]} \Omega$ is not only closed but even exact, and in fact it is the differential of the function $\Omega(Y, X)$. First, using the fact that Lie derivation satisfies a Leibnitz formula with respect to any natural bilinear operation on tensors (so in particular with respect to the interior product), $\mathcal{L}_{X}\left(i_{Y} \Omega\right)=i_{\left(\mathcal{L}_{X} Y\right)} \Omega+i_{Y}\left(\mathcal{L}_{X} \Omega\right)$. Thus, since $\mathcal{L}_{X} Y=[X, Y]$ and $\mathcal{L}_{X} \Omega=0, \mathcal{L}_{X}\left(i_{Y} \Omega\right)=i_{[X, Y]} \Omega$. Finally, since $d\left(i_{Y} \Omega\right)=0$, Cartan's formula for $\mathcal{L}_{X}\left(i_{Y} \Omega\right)$ gives $i_{[X, Y]} \Omega=d i_{X}\left(i_{Y} \Omega\right)=d(\Omega(Y, X))$. 
Remark. It is possible to prove Cartan's Formula by an ugly, brute force calculation of both sides, but there is also an elegant, no-sweat proof that I first learned from S. S. Chern (when I proudly showed him my version of the ugly proof). There is an important involutory automorphism $\omega \mapsto \bar{\omega}$ of the algebra $A$ of differential forms on a manifold. Namely, it is the identity on forms of even degree and is minus the identity on forms of odd degree. A linear map $\partial: A \rightarrow A$ is called an anti-derivation if $\partial(\lambda \omega)=\partial \lambda \wedge \omega+\bar{\lambda} \wedge \partial \omega$. It is of course well-known that the exterior derivative, $d$, is an anti-derivation (of degree +1 ), and an easy check shows that the interior product $i_{X}$ is an anti-derivation (of degree -1 ). Moreover, the anti-commutator of two anti-derivations is clearly a derivation, so that $\mathcal{L}_{X}$ and $d i_{X}+i_{X} d$ are both derivations of $A$, and hence to prove they are equal it suffices to check that they agree on a set of generators of $A$. But $A$ is generated by forms of degree zero (i.e., functions) and the differentials of functions, and it is obvious that $\mathcal{L}_{X}$ and $d i_{X}+i_{X} d$ agree on these.

We shall also have to deal with symplectic structures on infinite dimensional manifolds. In this case we still require that $\Omega$ is a closed form and we also still require that $\Omega$ is weakly non-degenerate, meaning that for each point $p$ of $P$, the map $v \mapsto i_{v} \Omega$ of $T P_{p}$ to $T P_{p}^{*}$ is injective. In finite dimensions this of course implies that $\Omega$ is strongly non-degenerate - meaning that the latter map is in fact an isomorphism - but that is rarely the case in infinite dimensions, so we will not assume it. Thus, if $F$ is a smooth function on $P$, it does not automatically follow that there is a symplectic gradient vector field $\boldsymbol{\nabla}_{s} F$ on $P$ satisfying $\Omega\left(\left(\boldsymbol{\nabla}_{s} F\right)_{p}, v\right)=$ $d F_{p}(v)$ for all $v$ in $T P_{p}$ - this must be proved separately. However, if a symplectic gradient does exist, then weak non-degeneracy shows that it is unique. In the infinite dimensional setting we call a function $F: P \rightarrow \mathbf{R}$ a Hamiltonian function if it has a symplectic gradient, and vector fields of the form $\nabla_{s} F$ will be called Hamiltonian vector fields. Obviously the space of Hamiltonian functions is linear, and in fact the formula $d(F G)=F d G+G d F$ shows that it is even an algebra, and that $\boldsymbol{\nabla}_{s}(F G)=F \boldsymbol{\nabla}_{s} G+G \boldsymbol{\nabla}_{s} F$. We shall call a vector field $X$ on $P$ symplectic if the 1 -form $i_{X} \Omega$ is closed but not necessarily exact, for as we have seen, this is the condition for the flow generated by $X$ to preserve $\Omega$.

Of course if $P$ is a vector space, the distinction between Hamiltonian and symplectic disappears: if $i_{X} \Omega$ is closed, then $H(p)=\int_{0}^{1} \Omega_{t p}\left(X_{t p}, p\right) d t$ defines a Hamiltonian function with $\nabla_{s} H=X$. Moreover, in this case it is usually straightforward to check if $i_{X} \Omega$ is closed. Given $u, v$ in $P$, consider them as constant vector fields on $P$, so that $[u, v]=0$. Then the formula $d \theta(u, v)=u(\theta(v))-v(\theta(u))-\theta([u, v])$ for the exterior derivative of a 1 -form shows that symmetry of $\left.\frac{d}{d t}\right|_{t=0} \Omega\left(X_{p+t u}, v\right)$ in $u$ and $v$ is necessary and sufficient for $i_{X} \Omega$ to be closed (and hence exact). In case $\Omega$ is a constant form (i.e., $\Omega_{p}(u, v)$ is independent of $p$ ), then $\left.\frac{d}{d t}\right|_{t=0} \Omega\left(X_{p+t u}, v\right)=$ $\Omega\left(\left(D X_{p}\right)(u), v\right)$, where $(D X)_{p}(u)=\left.\frac{d}{d t}\right|_{t=0} X_{p+t u}$ is the differential of $X$ at $p$. Since $\Omega$ is skew-symmetric in $u$ and $v$, this shows that if $\Omega$ is constant, then $X$ is Hamiltonian if and only if $(D X)_{p}$ is "skew-adjoint" with respect to $\Omega$.

If two smooth real-valued functions $F_{1}$ and $F_{2}$ on a symplectic manifold $P$ are Hamiltonian, i.e., if they have symplectic gradients $\nabla_{s} F_{1}$ and $\nabla_{s} F_{2}$, then they determine a third function on $P$, called their Poisson bracket, defined by:

$$
\left\{F_{1}, F_{2}\right\}=\Omega\left(\nabla_{s} F_{2}, \nabla_{s} F_{1}\right)
$$


The formula $i_{[X, Y]} \Omega=d(\Omega(Y, X))$ shows that the Poisson bracket is also a Hamiltonian function, and in fact

$$
\nabla_{s}\left\{F_{1}, F_{2}\right\}=\left[\nabla_{s} F_{1}, \nabla_{s} F_{2}\right] .
$$

What this formula says is that Hamiltonian functions $F: P \rightarrow \mathbf{R}$ are not only a commutative and associative algebra under pointwise product, but also a Lie algebra under Poisson bracket, and $F \mapsto \nabla_{s} F$ is a Lie algebra homomorphism of this Lie algebra onto the Lie algebra of Hamiltonian vector fields on $P$. In particular, we see that the Poisson bracket satisfies the Jacobi identity,

$$
\left\{\left\{F_{1}, F_{2}\right\}, F_{3}\right\}+\left\{\left\{F_{2}, F_{3}\right\}, F_{1}\right\}+\left\{\left\{F_{3}, F_{2}\right\}, F_{2}\right\}=0,
$$

and the Leibnitz Rule $\nabla_{s}(F G)=F \nabla_{s} G+G \nabla_{s} F$ gives:

$$
\left\{F_{1}, F_{2} F_{3}\right\}=\left\{F_{1}, F_{2}\right\} F_{3}+F_{2}\left\{F_{1}, F_{3}\right\},
$$

which we will also call the Leibnitz Rule.

Remark. A Poisson structure for a smooth manifold is defined to be a Lie algebra structure $\{F, G\}$ on the algebra of smooth functions that satisfies the Leibnitz Rule.

Since $\left\{F_{1}, F_{2}\right\}=\Omega\left(\nabla_{s} F_{2}, \nabla_{s} F_{1}\right)=d F_{2}\left(\nabla_{s} F_{1}\right)=\nabla_{s} F_{1}\left(F_{2}\right)$, we can interpret the Poisson bracket of $F_{1}$ and $F_{2}$ as the rate of change of $F_{2}$ along the solution curves of the vector field $\nabla_{s} F_{1}$. If we are considering some fixed Hamiltonian system $\frac{d x}{d t}=\nabla_{s} H_{x}$ on $P$, then we can write this as $\frac{d F}{d t}=\{H, F\}$, and we see that the vanishing of the Poisson bracket $\{H, F\}$ is the necessary and sufficient condition for $F$ to be a constant of the motion. By the Jacobi Identity, a corollary to this observation is that the Poisson Bracket of two constants of the motion is also a constant of the motion. And since $\{H, H\}=0, H$ itself is always a constant of the motion. (This is a proof of conservation of energy from the Hamiltonian point of view, and below we will also see how to prove Noether's Theorem in the Hamiltonian framework.)

Since the Poisson bracket is skew-symmetric, $\left\{F_{1}, F_{2}\right\}$ is zero if and only if $\left\{F_{2}, F_{1}\right\}$ is zero, and in this case we say that $F_{1}$ and $F_{2}$ are in involution. More generally $k$ Hamiltonian functions $F_{1}, \ldots, F_{k}$ are said to be in involution if all of the Poisson brackets $\left\{F_{i}, F_{j}\right\}$ vanish. Note that since $\nabla_{s}\left\{F_{i}, F_{j}\right\}=\left[\nabla_{s} F_{i}, \nabla_{s} F_{j}\right]$, if the $F_{i}$ are in involution then the vector fields $\nabla_{s} F_{i}$ commute, i.e., $\left[\nabla_{s} F_{i}, \nabla_{s} F_{j}\right]=0$, or equivalently the flows they generate commute. In particular we see that if $F_{1}, \ldots, F_{n}$ are in involution and if each $\nabla_{s} F_{i}$ generates a one-parameter group of diffeomorphisms $\phi_{t}^{i}$ of $P$, then $\left(t_{1}, \ldots, t_{n}\right) \mapsto \phi_{t_{1}}^{1} \circ \phi_{t_{2}}^{2} \circ \ldots \circ \phi_{t_{n}}^{n}$ defines a symplectic action of the abelian group $\mathbf{R}^{n}$ on $P$.

Suppose $P$ is a symplectic manifold of dimension $2 n$ and that there exist $n$ functions $F_{i}$ such that the $d F_{i}$ are everywhere linearly independent. If the functions $F_{i}$ are in involution with each other and with a function $H$, then the so-called ArnoldLiouville Theorem ([Ar], Chapter 10) states that the Hamiltonian system $\nabla_{s} H$ is completely integrable in the sense mentioned earlier, i.e., there exist action-angle variables $q_{1}, \ldots, q_{n}, p_{1}, \ldots, p_{n}$. In fact, complete integrability of a $2 n$ dimensional Hamiltonian system is often defined as the existence of $n$ functionally independent constants of the motion in involution.

This leads naturally to two interesting problems: finding ways to construct symplectic manifolds with lots of functions in involution, and determining whether a given Hamiltonian system is completely integrable. In the late 1970's M. Adler 
[Ad], B. Kostant [Kos], and W. Symes [Sy] independently and nearly simultaneously found a beautiful approach to the first question using certain special splittings of Lie algebras. For excellent surveys of finite dimensional completely integrable systems see $[\mathrm{AdM}]$ and $[\mathrm{Pe}]$. The Adler-Kostant-Symes Theorem is explained in detail in both of these references, and we shall not discuss it further here, except to note that it is closely related to an earlier method of Peter Lax [La1] that will be one of our main tools in later sections, and that, as Adler's paper showed, the Adler-Kostant-Symes Theorem also applies to infinite dimensional systems. In fact Adler's paper applied the method to the KdV equation, and later many other PDE were treated by the A-K-S approach in [Dr], [DS], [RS], [Se1], [Se2], and [Te2].

As for the second problem, there is no magic test to check if a given system is completely integrable, and the principal technique is to try to show that it can be manufactured using the Adler-Kostant-Symes method. In fact, one often hears it said that "all known completely integrable systems arise in this way".

If a symplectic structure $\Omega$ is "exact"-i.e., if $\Omega=d \omega$ for some 1 -form $\omega$ on $P$ (as we saw was the case for a cotangent bundle) and if a vector field $X$ not only preserves $\Omega$ but even preserves $\omega$, then Cartan's formula gives $0=\mathcal{L}_{X} \omega=d i_{X} \omega+i_{X} \Omega$; so if we define $X^{\omega}=-i_{X} \omega=-\omega(X)$, then $\nabla_{s}\left(X^{\omega}\right)=X$. If $Y$ is a second such vector field on $P$, then a computation completely analogous to that for $i_{[X, Y]} \Omega$ above (replacing $\Omega$ by $\omega$ ) gives $[X, Y]^{\omega}=\omega([Y, X])=i_{[Y, X]} \omega=i_{Y} d\left(i_{X} \omega\right)=-d X^{\omega}(Y)=$ $-d X^{\omega}\left(\nabla_{s} Y^{\omega}\right)=\left\{X^{\omega}, Y^{\omega}\right\}$. Thus $X \mapsto X^{\omega}$ is a Lie algebra homomorphism inverse to $F \mapsto \nabla_{s} F$ from the Lie algebra of vector fields preserving $\omega$ to the Lie algebra of Hamiltonian functions under Poisson bracket.

In particular going back to Newton's Equations on our configuration space $\mathcal{C}$, we see that if $X$ is a Killing vector field on $\mathcal{C}$ such that $X U=0$, then $\omega(X)$ is a constant of the motion for Newton's Equations. It is easy to see that $\omega(X)$ is just the conjugate momentum of $X$, so this gives a proof of Noether's Principle in the Hamiltonian framework.

6. Examples of Classical Mechanical Systems. While any choice of potential function $U$ on any Riemannian manifold $\mathcal{C}$ defines a "Classical Mechanical System", in some generalized sense, this name is often reserved for certain more special cases that arise from physical considerations.

One important and interesting class of examples describes the motion of rigid bodies or "tops" with no external forces acting. Here the configuration space $\mathcal{C}$ is the rotation group $\mathbf{S O}(3)$, while the metric tensor (also called the inertia tensor in this case) is any left-invariant metric on $\mathcal{C}$, and $U=0$. We refer the reader to any book on Classical Mechanics (e.g., [AbM], [Ar]) for a discussion of these examples, but be warned that the full theory is covered in a multi-volume treatise [KS]. An excellent recent book is $[\mathrm{Au}]$.

A second important class of examples, usually referred to as "particle mechanics", describes the motion under mutual forces of $N$ particles in the Euclidean space $\mathbf{R}^{k}$ (where usually $k=1,2$, or 3 ). In this case $\mathcal{C}=\left(\mathbf{R}^{k}\right)^{N}$, a point $x=\left(x_{1}, \ldots, x_{N}\right)$ of $\mathcal{C}$ representing the positions of $N$ particles. For an important subclass, the force on each particle is the sum of forces exerted on it by the remaining particles. In this case the potential $U$ is a function of the distances $r_{i j}=\left\|x_{i}-x_{j}\right\|$ separating the particles. It follows that the Lie group $G$ of Euclidean motions of $\mathbf{R}^{k}$ is a group of symmetries, so the conjugate momenta of the Lie algebra of $G$ give $k$ 
linear momenta (from the translations) and $k(k-1) / 2$ angular momentum (from the rotations) that are conserved quantities.

A simple but important example from particle mechanics is the "harmonic oscillator". Here $k=N=1$, so $\mathcal{C}=\mathbf{R}$, the metric on $T \mathcal{C}=\mathbf{R} \times \mathbf{R}$ is given by $\|(x, v)\|^{2}=m v^{2}$ (where $m$ is the mass of the oscillator) and $U(x)=\frac{1}{2} k x^{2}$, where $k>0$ is the so-called spring constant of the oscillator. This models a particle that is in equilibrium at the origin, but which experiences a Hooke's Law linear "restoring force" of magnitude $-k x$ directed towards the origin when it is at the point $x$ in $\mathcal{C}$. Newton's Equation of motion is $m \ddot{x}=-k x$, and the solutions are of the form $x(t)=A \cos \left(\omega\left(t-t_{0}\right)\right)$, where the angular frequency $\omega$ is $\sqrt{k / m}$. The Hamiltonian formulation of the harmonic oscillator is given in terms of canonical variables $q=x$ and $p=m(d x / d t)$ by $H(q, p)=\frac{1}{2}\left(p^{2} / m+k q^{2}\right)$. Note that $P=\frac{1}{2}\left(p^{2}+m k q^{2}\right)$ and $Q=\arctan (p / q \sqrt{m k})$ define action-angle variables for the harmonic oscillator.

Only notationally more complicated is the case of $N$ uncoupled harmonic oscillators, with masses $m_{1}, \ldots, m_{N}$ and spring constant $k_{1}, \ldots, k_{N}$. Now $\mathcal{C}=\mathbf{R}^{N}$, the metric on $T \mathcal{C}=\mathbf{R}^{N} \times \mathbf{R}^{N}$ is given by $\|(x, v)\|^{2}=\sum_{i} m_{i} v_{i}^{2}$, and the potential function is $U(x)=\frac{1}{2} \sum_{i} k_{i} x_{i}^{2}$. Newton's Equations are $m_{i} \ddot{x}_{i}=-k_{i} x_{i}$ with the solutions $x_{i}(t)=A_{i} \cos \left(\omega_{i}\left(t-t_{0}^{i}\right)\right)$, where $\omega_{i}=\sqrt{k_{i} / m_{i}}$. The Hamiltonian for this example is $H(q, p)=\sum_{i} \frac{1}{2}\left(p_{i}^{2} / m_{i}+k q_{i}^{2}\right)$. Note that not only is the total Hamiltonian, $H$, a constant of the motion, but so also are the $N$ partial Hamiltonians, $H_{i}(q, p)=\frac{1}{2}\left(p_{i}^{2} / m_{i}+k q_{i}^{2}\right)$-i.e., the sum of the kinetic plus potential energy of each individual oscillator is preserved during the time evolution of any solution. In this case we get one pair of action-angle variables from the action-angle variables for each of the individual harmonic oscillators, so it is again completely integrable.

A seemingly more complicated example is the case of $N$ coupled harmonic oscillators. Starting from the previous example, we imagine adding Hooke's Law springs with spring constants $K_{i j}$ joining the $i$-th and $j$-th particles. The force on the $i$-th particle is now $F_{i}=-k_{i} x_{i}-K_{i j}\left(x_{i}-x_{j}\right)$, so we can take as our potential function $U(x)=\frac{1}{2} \sum k_{i} x_{i}^{2}+\frac{1}{2} \sum_{i j} K_{i j}\left(x_{i}-x_{j}\right)^{2}$. Notice that this is clearly a positive definite quadratic form, so without loss of generality we can consider the somewhat more general potential function $U(x)=\frac{1}{2} \sum_{i j} k_{i j} x_{i} x_{j}$, where $k_{i j}$ is a positive definite symmetric matrix. Newton's Equations are now $m_{i} \ddot{x}_{i}=-\sum_{j} k_{i j} x_{j}$. Because of the off-diagonal elements of $k_{i j}$ (the so-called "coupling constants") Newton's Equations no longer have separated variables, and integrating them appears much more difficult. This is of course an illusion; all that is required to reduce this case to the case of uncoupled harmonic oscillators is to diagonalize the quadratic form that gives the potential energy, i.e., find an orthonormal basis $e_{i}, \ldots, e_{n}$ such that if $y=y_{1} e_{i}+\ldots+y_{n} e_{n}$, then $U(y)=\frac{1}{2} \sum_{i} \lambda_{i} y_{i}^{2}$. The solutions of Newton's Equations are now all of the form $\sum_{i} A_{i} \cos \left(\sqrt{\lambda_{i}}\left(t_{i}-t_{0}^{i}\right)\right) e_{i}$. Solutions for which one $A_{i}$ is non-zero and all the others are zero are referred to as "normal modes" of the coupled harmonic oscillator system. Since the coupled harmonic oscillator system is just the uncoupled system in disguise, we see that it also is completely integrable. Moreover, when we express a solution $x(t)$ of Newton's Equations as a sum of normal modes, then not only is the kinetic energy plus the potential energy of $x(t)$ a constant of the motion, but also the kinetic plus the potential energy of each of these normal modes is also a constant of the motion.

There are two properties of the coupled harmonic oscillators that make it an exceptionally important model system. First, it is exactly and explicitly solvable, 
and secondly, as we shall see in the next section, it is an excellent first approximation to what happens in an arbitrary system near a so-called "vacuum solution". i.e., a stable equilibrium.

7. Physics Near Equilibrium. Physical systems are normally close to equilibrium, so it is important to analyze well what happens in the phase space of a physical system in the near neighborhood of an equilibrium point.

We shall assume that our system is described as above by a potential $U$ on a configuration space $\mathcal{C}$. By an "equilibrium point" we mean a point $p$ of $\mathcal{C}$ that is not just a critical point of $U$, but in fact a non-degenerate local minimum. Since $U$ is determined only up to an additive constant, we can assume that $U(p)=0$. Since $\nabla U$ vanishes at $p$, it is clear that $\sigma(t)=p$ is a solution of Newton's Equations, and physicists sometimes refer to such a solution as a "vacuum solution".

By a famous result of Marston Morse, we can find local coordinates $y_{1}, \ldots, y_{n}$ in a neighborhood $O$ of $p$ and centered at $p$ such that $U(q)=\sum_{i} y_{i}(q)^{2}$, so that $N(\epsilon)=\{q \in O \mid U(q)<\epsilon\}$ is a neighborhood basis for $p$. It follows that a vacuum solution is stable, i.e., a solution of Newton's Equations with initial conditions sufficiently close to those of a vacuum solution will remain close to the vacuum solution for all time. To be precise, suppose $\gamma(t)$ is a solution of Newton's Equations such that $\gamma(0)$ is in $N\left(\frac{1}{2} \epsilon\right)$ and $K\left(\gamma^{\prime}(0)\right)<\frac{1}{2} \epsilon$. Then $U(\gamma(0))+K\left(\gamma^{\prime}(0)\right)<\epsilon$, so that, by conservation of total energy, $U(\gamma(t))+K\left(\gamma^{\prime}(t)\right)<\epsilon$ for all $t$, and since $K$ is non-negative, $U(\gamma(t))<\epsilon$ for all $t$, i.e., the solution $\gamma(t)$ remains inside $N(\epsilon)$.

But we can be much more precise about the nature of these solutions that are near the vacuum. To simplify the exposition somewhat we will make the (inessential) assumption that the metric on $\mathcal{C}$ is flat - as it usually is in particle mechanics. Then we can choose orthogonal coordinates $x_{1}, \ldots, x_{n}$ centered at $p$ that simultaneously diagonalizes both the kinetic energy and the Hessian matrix of $U$ at $p$, and the assumption that $p$ is a non-degenerate local minimum just means that the diagonal elements, $k_{i}$, of the Hessian are positive. (The diagonal elements, $m_{i}$, of the kinetic energy are of course also positive and have the interpretations of masses.) Thus, by Taylor's Theorem, $U(x)=\frac{1}{2} \sum_{j} k_{j} x_{j}^{2}+\frac{1}{6} \sum_{j k l} a_{j k l}(x) x_{j} x_{k} x_{l}$, where the functions $a_{j k l}(x)$ are smooth and symmetric in their last two indices, and Newton's Equations take the form:

$$
m_{i} \frac{d^{2} x_{i}(t)}{d t^{2}}=-k_{i} x_{i}-\sum_{j k} a_{i j k}(x) x_{k} x_{l}+O\left(\|x\|^{3}\right) .
$$

(For later reference, we note that if we adopt a Hamiltonian viewpoint and move to the cotangent bundle using the Legendre transform, then in the canonical symplectic coordinates associated to $x_{1}, \ldots, x_{n}$, the kinetic energy $K$ is given by $K=\frac{1}{2} \sum_{i} \frac{p_{i}^{2}}{m_{i}}$, the potential energy is $U=\frac{1}{2} \sum_{j} k_{j} q_{j}^{2}+\frac{1}{6} \sum_{j k l} a_{j k l}(q) q_{j} q_{k} q_{l}$, and the Hamiltonian is $H=K+U$.)

The system of uncoupled harmonic oscillators obtained by dropping the nonlinear terms is called the "linearized system" (at the given equilibrium $p$ ), and its normal modes are referred to by physicists as the "degrees of freedom" of the system.

An obvious question is, "To what extent do solutions of the linearized system approximate those of the full system?" One answer is easy, and no surpriseGronwal's Inequality implies that, as the initial position tends to $p$ and the initial velocity tends to zero, a solution of the linearized equation approximates that of the full equation better and better, and for a longer period of time. 
A more subtle, but also more interesting, question is, "How will the kinetic and potential energy of a solution become distributed, on average, among the various degrees of freedom of the full system?" It is not difficult to give a precise formulation of this question. The kinetic energy in the $i$-th mode is clearly $K_{i}=\frac{1}{2} \frac{p_{i}^{2}}{m_{i}}$, and it is natural to assign to the $i$-th mode the potential energy $U_{i}=\frac{1}{2} k_{i} q_{i}^{2}+\frac{1}{6} \sum_{k l} a_{i k l}(q) q_{i} q_{k} q_{l}$. Then $H_{i}=K_{i}+U_{i}$ is that part of the total energy in the $i$-th mode, and the total energy $H$ is just the sum of these $H_{i}$. We know that for the linearized system each of the $H_{i}$ is a constant of the motion; that is, $H_{i}$ is constant along any solution of Newton's Equations. But it is easy to see that cannot be true for the full system, and energy will in general flow between the normal modes because of the nonlinear coupling between them. The question is, will the "average behavior" of the $H_{i}$ and $K_{i}$ have some predictable relationship over large time intervals.

To make the concept of "average" precise, given any function $F: T \mathcal{C} \rightarrow \mathbf{R}$, define its "time average", $\bar{F}$, along a given solution $x(t)$ by: $\bar{F}=\lim _{T \rightarrow \infty} \frac{1}{T} \int_{-T}^{T} F(\dot{x}(t)) d t$. Then, what can we say about the time averages of the above partial energy functions and their relations to each other? Of course a first question is whether the limit defining the time average really exists, and this is already a non-trivial point. Fortunately, as we shall see in the next section, it is answered by the "Individual Ergodic Theorem" of G. D. Birkhoff [Bi], according to which the time average will exist for "almost all" initial conditions.

Starting in the late nineteenth century, physicists such as Maxwell, Boltzmann, and Gibbs developed a very sophisticated theory of statistical mechanics that gave convincing explanations for (and good predictions of) the behavior of large assemblages of molecules. The theoretical foundations for this theory were based on just such time averages and their hypothesized equality with another kind of average that is easier to investigate, so-called "space averages", or "microcanonical averages". As we will see, the space average of the kinetic energy in each normal mode is the same - a fact referred to as "equipartition of energy". This important fact is the very basis for the definition of temperature in statistical mechanics. Namely, for a system near equilibrium, if the absolute temperature is $T$, then the average kinetic energy in each degree of freedom is $\frac{k T}{2}$, where $k$ is the so-called Boltzmann constant.

But it is the time averages of the kinetic energy that should really determine the temperature, and if energy equipartition holds for time averages, and if the system is experimentally started in one of its normal modes and is then followed in time, one should see an equilibration take place, in which the kinetic energy should gradually flow out of the original single mode in which it was concentrated and become equally divided (on average) among all the various degrees of freedom of the system. Because of the above relation between temperature and equipartition of energy, this hypothesized equilibration process is referred to as "thermalization". Intuitively speaking, this refers to the transformation of the large scale motion of the system in a single mode into "heat", i.e., lots of tiny fluctuating bits of energy of amount $\frac{k T}{2}$ in each of the many degrees of freedom.

It should now be clear why physicists placed so much emphasis on proving the supposed equality of the time average and the microcanonical average, but mathematically this proved to be a highly intractible problem. There were heuristic proofs, based on vague physical reasoning, and also semi-rigorous arguments based on so-called "ergodic hypotheses". The latter were assumptions to the effect that 
the solution curves would wander on an energy surface in a sufficiently space-filling way (ergodic comes from the Greek word for energy). Unfortunately these ergodicity assumptions were vague and in certain cases topologically impossible, and it was only with the development of measure theory that von Neumann and Birkhoff were able to state the precise condition ("metric transitivity") under which one could prove that time and space averages must necessarily coincide.

Nevertheless, physicists were morally convinced of the correctness of the timeaverage based concept of thermalization; so much so that when Fermi, Pasta, and Ulam undertook the numerical experiments that we will consider later, they stated that their goal was not so much to discover if there would be be thermalization, but rather to discover experimentally what the rate of approach to thermalization would be!

For those readers who are interested, we will provide more of the mathematical details concerning equipartition of energy in the next section.

8. Ergodicity and Thermalization. Let $P$ be a symplectic manifold (say of dimension $2 n$ ) with symplectic 2 -form $\Omega$, and let $H$ denote a Hamiltonian function on $P$, generating a symplectic flow $\phi_{t}$; i.e., the infinitesimal generator of $\phi_{t}$ is $\nabla_{s} H$, the symplectic gradient of $H$. As we have seen, this implies that the flow $\phi_{t}$ preserves the symplectic structure, and also that $H$ is a "constant of the motion", meaning that it is constant along every orbit, $\phi_{t}(p)$, or equivalently, that the constant energy hypersurfaces $\Sigma_{c}$ (defined by $H=c$ ) are invariant under the flow. In classical examples, the Hamiltonian is usually bounded below and proper (so that all the $\Sigma_{c}$ are compact) and we shall assume this in what follows. Since $H$ is only defined up to an additive constant, we can assume the minimum value of $H$ is zero.

The $2 n$-form $\Omega^{n}$ defines a measure $d \mu$ on $P$ (the Liouville measure), and this is of course invariant under the flow. We can factor $\Omega^{n}$ as $\Omega^{n}=\lambda \wedge d H$, and the $2 n-1$ form $\lambda$ is uniquely determined modulo the ideal generated by $d H$, so it induces a unique measure on each energy hypersurface $\Sigma_{c}$. We will denote these measures by $d \sigma$, and they are of course likewise invariant under the flow. Since $\Sigma_{c}$ is compact, its total measure, $\sigma(c)$, is finite, and so, for any integrable function $f$ on $\Sigma_{c}$, we can define its spatial average by $\hat{f}=\sigma(c)^{-1} \int_{\Sigma_{c}} f(x) d \sigma(x)$. (This is the quantity called the "microcanonical average" of $f$ in statistical mechanics.) We note that these measure $d \sigma$ are canonically determined in terms of the Liouville form, $\Omega^{n}$, and the Hamiltonian function $H$, so if $\psi$ is any diffeomorphism of $P$ that preserves $\Omega^{n}$ and preserves $H$, then $\psi$ will also preserve the $d \sigma$ and hence all microcanonical averages, i.e., if $g=f \circ \psi$, then $\hat{g}=\hat{f}$.

We return now to the question of "equipartition of energy". We assume that we have canonical variables $\left(p_{1}, \ldots, p_{n}, q_{1}, \ldots, q_{n}\right)$ in $P$ in which $H$ takes the classical form $H=K+U$ where $K=\frac{1}{2} \sum_{i} \frac{p_{i}^{2}}{m_{i}}$, and $U$ is a function of the $q_{i}$ with a nondegenerate local minimum, zero, at the origin. (It follows that for small $c$ the energy surfaces $\Sigma_{c}$ are not only compact, but are in fact topologically spheres.) Since the $p$ 's and $q$ 's are canonical, $\Omega$ has the standard Darboux form $\sum_{i} d p_{i} \wedge d q_{i}$, and so the Liouville $2 n$-form is just $d p_{1} \wedge d q_{1} \wedge \ldots \wedge d p_{n} \wedge d q_{n}$, giving Lebesgue measure as the Liouville measure in these coordinates. Our goal is to prove that if $K_{i}=\frac{p_{i}^{2}}{m_{i}}$, then the microcanonical averages $\hat{K}_{i}, i=1, \ldots, n$ (over any fixed energy surface $\Sigma_{c}$ ) are all the same. Without loss of generality we can assume that $i=1$ and $j=2$, and by the remark above it will suffice to find a diffeomorphism $\psi$ that preserves 
$H=K+U$ and the Liouville form such that $K_{2}=K_{1} \circ \psi$. In fact, define

$$
\psi\left(p_{1}, p_{2}, p_{3}, \ldots, p_{n}, q_{1}, \ldots, q_{n}\right)=\left(\alpha p_{2}, \alpha^{-1} p_{1}, p_{3}, \ldots, p_{n}, q_{1}, \ldots, q_{n}\right),
$$

where $\alpha=\sqrt{m_{1} / m_{2}}$. Now, while $\psi$ is clearly not symplectic, it just as clearly does preserve the Liouville form. Moreover a trivial calculation shows that $K_{2}=K_{1} \circ \psi$ and $K_{1}=K_{2} \circ \psi$, while $K_{i}=K_{i} \circ \psi$ for $i>2$. Since $K=\sum_{i} K_{i}, K=K \circ \psi$. Since $U$ is a function of the $q$ 's and not the $p$ 's, $U=U \circ \psi$, so $H=H \circ \psi$ also, and this completes the proof that $\hat{K}_{2}=\hat{K}_{1}$.

There is an important corollary of the above proof. Suppose that we can write the potential energy $U$ as the sum of $n$ functions $U_{i}$, and let us define $H_{i}=K_{i}+U_{i}$. You should think of $U_{i}$ as representing the "potential energy in the $i$-th normal mode", and similarly $H_{i}$ represents the part of the total energy that is "in" the $i$-th normal mode. In applications where the potential $U$ describes an interaction between identical particles, these partial potentials will satisfy $U_{1}\left(q_{1}, q_{2}, \ldots, q_{n}\right)=$ $U_{2}\left(q_{2}, q_{1}, \ldots, q_{n}\right)$, and similarly for other pairs of indices. (For the example of the preceding section, we note that these conditions will be satisfied if the "spring constants" $k_{i}$ are all equal and if the functions $a_{i j k}$ are symmetric in all three indices.) We remark that, in particular, these conditions are satisfied for the FermiPasta-Ulam Lattice that we will consider shortly. If we now redefine $\psi$ above to simply interchange $q_{i}$ and $q_{j}$, then the same argument as before shows that $\hat{U}_{i}=\hat{U}_{j}$, and so of course we also have $\hat{H}_{i}=\hat{H}_{j}$. In words, for such systems not only kinetic energy per mode, but also potential and total energies per mode are "equi-partitioned", in the sense that their microcanonical averages are equal.

Next recall that for $p$ in $\Sigma_{c}$ we define the time average of $f$ on the orbit of $p$ by:

$$
\bar{f}(p)=\lim _{T \rightarrow \infty} \frac{1}{T} \int_{-T}^{T} f\left(\phi_{t}(p)\right) d t,
$$

provided the limit exists. G. D. Birkhoff's Individual Ergodic Theorem ([Bi]) states that $\bar{f}(p)$ is defined for almost all $p$ in $\Sigma_{c}$, and then clearly $\bar{f}$ is invariant under the flow. It is moreover again an integrable function on $\Sigma_{c}$ with the same spatial average as $f$ itself. It is then easily seen that the following four conditions are equivalent:

1) For every integrable function $f$ on $\Sigma_{c}$, its time average $\bar{f}$ is constant (and hence equal to its spatial average).

2) Every measurable subset of $\Sigma_{c}$ that is invariant under the flow either has measure zero or has measure $\sigma(c)$.

3) If an integrable function on $\Sigma_{c}$ is constant on each orbit of the flow, then it is constant (almost everywhere) on $\Sigma_{c}$.

4) Given two subsets $E_{1}$ and $E_{2}$ of $\Sigma_{c}$ having positive measure, some translate $\phi_{t}\left(E_{1}\right)$ of $E_{1}$ meets $E_{2}$ in a set of positive measure,

and if these equivalent conditions are satisfied, then the flow is said to be ergodic or metrically transitive on $\Sigma_{c}$.

By choosing $f$ to be the characteristic function of an open set $O$, we see from 1) that ergodicity implies that the motion has a "stochastic" nature - that is, the fraction of time that an orbit spends in $O$ is equal to the measure of $O$ (so in particular almost all orbits are dense in $\Sigma_{c}$ ). This implies that (apart from $\Sigma_{c}$ itself) there cannot exist any stable fixed point, periodic orbit, or more general 
stable invariant set. To put it somewhat more informally, orbits on an ergodic $\Sigma_{c}$ cannot exhibit any simple asymptotic behavior.

Note that any function of a constant of the motion will again be a constant of the motion - and in particular any function of $H$ is a constant of the motion. There may of course be constants of the motion that are functionally independent of $H$. But if the flow is ergodic on every energy surface, then it follows from 3) that any constant of the motion will be constant on each level set of $H$-which is just to say that it is a function of $H$. This shows that Hamiltonian systems with many independent constants of the motion (and in particular completely integrable systems) are in some sense at the opposite extreme from ergodic systems.

So what is the status of the old belief that a "generic" (in some suitable sense) Hamiltonian system should be ergodic on each energy surface? On the one hand, Fermi $[\mathrm{Fe}]$ proved a result that points in this direction. And there is a famous result of Oxtoby and Ulam [OU] to the effect that in the set of all measure preserving homeomorphisms of an energy surface, those that are metrically transitive are generic in the sense of category. But the measure preserving diffeomorphisms of an energy surface are themselves only a set of first category in the measure preserving homeomorphisms, so the Oxtoby-Ulam theorem is not particularly relevant to this question. In fact, the KAM (Kolmagorov-Arnold-Moser) Theorem ([Ar], Appendix 8) shows that any Hamiltonian flow that is sufficiently close to a completely integrable system in a suitable $C^{k}$ topology will have a set of invariant tori of positive Liouville measure, and so cannot be ergodic. Indeed, proving rigorously that any particular Hamiltonian system is ergodic is quite difficult. For some examples of such theorems see $[\mathrm{AA}]$.

\section{Origins of Soliton Theory}

Perhaps the single most important event leading up to the explosive growth of soliton mathematics in the last decades was a seemingly innocuous computer computation, carried out by Enrico Fermi, John Pasta, and Stanislaw Ulam in 1954-55, on the Los Alamos MANIAC computer. (Originally published as Los Alamos Report LA1940 (1955) and reprinted in [FPU].)

1. The Fermi-Pasta-Ulam Experiments. The following quotation is taken from Stanislaw Ulam's autobiography, Adventures of a Mathematician [Ul].

Computers were brand new; in fact the Los Alamos Maniac was barely finished .... As soon as the machines were finished, Fermi, with his great common sense and intuition, recognized immediately their importance for the study of problems in theoretical physics, astrophysics, and classical physics. We discussed this at length and decided to formulate a problem simple to state, but such that a solution would require a lengthy computation which could not be done with pencil and paper or with existing mechanical computers .... [W] e found a typical one ... the consideration of an elastic string with two fixed ends, subject not only to the usual elastic force of stress proportional to strain, but having, in addition, a physically correct nonlinear term .... The question was to find out how ... the entire motion would eventually thermalize ....

John Pasta, a recently arrived physicist, assisted us in the task of flow diagramming, programming, and running the problem on the Maniac .... 
The problem turned out to be felicitously chosen. The results were entirely different qualitatively from what even Fermi, with his great knowledge of wave motion, had expected.

What Fermi, Pasta, and Ulam (FPU) were trying to do was to verify numerically a basic article of faith of statistical mechanics; namely the belief that if a mechanical system has many degrees of freedom and is close to a stable equilibrium, then a generic nonlinear interaction will "thermalize" the energy of the system, i.e., cause the energy to become equidistributed among the normal modes of the corresponding linearized system. In fact, Fermi believed he had demonstrated this fact in [Fe]. Equipartition of energy among the normal modes is known to be closely related to the ergodic properties of such a system, and in fact FPU state their goal as follows: "The ergodic behavior of such systems was studied with the primary aim of establishing, experimentally, the rate of approach to the equipartition of energy among the various degrees of freedom of the system."

FPU make it clear that the problem that they want to simulate is the vibrations of a "one-dimensional continuum" or "string" with fixed end-points and nonlinear elastic restoring forces, but that "for the purposes of numerical work this continuum is replaced by a finite number of points ... so that the PDE describing the motion of the string is replaced by a finite number of ODE." To rephrase this in the current jargon, FPU study a one-dimensional lattice of $N$ oscillators with nearest neighbor interactions and zero boundary conditions. (For their computations, FPU take $N=64$.)

We imagine the original string to be stretched along the $x$-axis from 0 to its length $\ell$. The $N$ oscillators have equilibrium positions $p_{i}=i h, i=0, \ldots, N-1$, where $h=\ell /(N-1)$ is the lattice spacing, so their positions at time $t$ are $X_{i}(t)=p_{i}+$ $x_{i}(t)$, (where the $x_{i}$ represent the displacements of the oscillators from equilibrium). The force attracting any oscillator to one of its neighbors is taken as $k\left(\delta+\alpha \delta^{2}\right)$, $\delta$ denoting the "strain", i.e., the deviation of the distance separating these two oscillators from their equilibrium separation $h$. (Note that when $\alpha=0$ this is just a linear Hooke's law force with spring constant $k$.) The force acting on the $i$-th oscillator due to its right neighbor is $F(x)_{i}^{+}=k\left[\left(x_{i+1}-x_{i}\right)+\alpha\left(\left(x_{i+1}-x_{i}\right)^{2}\right]\right.$, while the force acting on it due to its left neighbor is $F(x)_{i}^{-}=k\left[\left(x_{i-1}-x_{i}\right)-\alpha\left(\left(x_{i-1}-\right.\right.\right.$ $\left.x_{i}\right)^{2}$. Thus the total force acting on the $i$-th oscillator will be the sum of these two forces, namely: $F(x)_{i}=k\left(x_{i+1}+x_{i-1}-2 x_{i}\right)\left[1+\alpha\left(x_{i+1}-x_{i-1}\right)\right]$, and assuming that all of the oscillators have the same mass, $m$, Newton's equations of motion read:

$$
m \ddot{x}_{i}=k\left(x_{i+1}+x_{i-1}-2 x_{i}\right)\left[1+\alpha\left(x_{i+1}-x_{i-1}\right)\right],
$$

with the boundary conditions $x_{0}(t)=x_{N-1}(t)=0$. In addition, FPU looked at motions of the lattice that start from rest, i.e., they assumed that $\dot{x}_{i}(0)=0$, so the motion of the lattice is completely specified by giving the $N-2$ initial displacements $x_{i}(0), i=1, \ldots, N-2$. We shall call this the FPU initial value problem (with initial condition $\left.x_{i}(0)\right)$.

It will be convenient to rewrite Newton's equations in terms of parameters that refer more directly to the original string that we are trying to model. Namely, if $\rho$ denotes the density of the string, then $m=\rho h$, while if $\kappa$ denotes the Young's modulus for the string (i.e., the spring constant for a piece of unit length), then $k=\kappa / h$ will be the spring constant for a piece of length $h$. Defining $c=\sqrt{\kappa / \rho}$ we 
can now rewrite Newton's equations as:

$$
\ddot{x}_{i}=c^{2}\left(\frac{x_{i+1}+x_{i-1}-2 x_{i}}{h^{2}}\right)\left[1+\alpha\left(x_{i+1}-x_{i-1}\right)\right],
$$

and in this form we shall refer to them as the FPU Lattice Equations. We can now "pass to the continuum limit"; i.e., by letting $N$ tend to infinity (so $h$ tends to zero) we can attempt to derive a PDE for the function $u(x, t)$ that measures the displacement at time $t$ of the particle of string with equilibrium position $x$. We shall leave the nonlinear case for later, and here restrict our attention to the linear case, $\alpha=0$. If we take $x=p_{i}$, then by definition $u(x, t)=x_{i}(t)$, and since $p_{i}+h=p_{i+1}$ while $p_{i}-h=p_{i-1}$, with $\alpha=0$ the latter form of Newton's equations gives:

$$
u_{t t}(x, t)=c^{2} \frac{u(x+h, t)+u(x-h, t)-2 u(x, t)}{h^{2}} .
$$

By Taylor's formula:

$$
f(x \pm h)=f(x) \pm h f^{\prime}(x)+\frac{h^{2}}{2 !} f^{\prime \prime}(x) \pm \frac{h^{3}}{3 !} f^{\prime \prime \prime}(x)+\frac{h^{4}}{4 !} f^{\prime \prime \prime \prime}(x)+O\left(h^{5}\right)
$$

and taking $f(x)=u(x, t)$ gives:

$$
\frac{u(x+h, t)+u(x-h, t)-2 u(x, t)}{h^{2}}=u_{x x}(x, t)+\left(\frac{h^{2}}{12}\right) u_{x x x x}(x, t)+O\left(h^{4}\right) ;
$$

so letting $h \rightarrow 0$, we find $u_{t t}=c^{2} u_{x x}$, i.e., $u$ satisfies the linear wave equation, with propagation speed $c$ (and of course the boundary conditions $u(0, t)=u(\ell, t)=0$, and initial conditions $\left.u_{t}(x, 0)=0, u(x, 0)=u_{0}(x)\right)$.

This is surely one of the most famous initial value problems of mathematical physics, and nearly every mathematician sees a derivation of both the d'Alembert and Fourier version of its solution early in their careers. For each positive integer $k$ there is a normal mode or "standing wave" solution:

$$
u_{k}(x, t)=\cos \left(\frac{k \pi c t}{\ell}\right) \sin \left(\frac{k \pi x}{\ell}\right),
$$

and the solution to the initial value problem is $u(x, t)=\sum_{k=1}^{\infty} a_{k} u_{k}(x, t)$ where the $a_{k}$ are the Fourier coefficients of $u_{0}$ :

$$
a_{k}=\frac{2}{l} \int_{0}^{\ell} u_{0}(x) \sin \left(\frac{k \pi x}{\ell}\right) d x .
$$

Replacing $x$ by $p_{j}=j h$ in $u_{k}(x, t)$ (and using $\ell=(N-1) h$ ) we get functions

$$
\xi_{j}^{(k)}(t)=\cos \left(\frac{k \pi c t}{(N-1) h}\right) \sin \left(\frac{k j \pi}{N-1}\right),
$$

and it is natural to conjecture that these will be the normal modes for the FPU initial value problem (with $\alpha=0$ of course). This is easily checked using the addition formula for the sine function. It follows that, in the linearized case, the solution to the FPU initial value problem with initial conditions $x_{i}(0)$ is given explicitly by $x_{j}(t)=\sum_{k=1}^{N-2} a_{k} \xi_{j}^{(k)}(t)$, where the Fourier coefficients $a_{k}$ are determined from the formula:

$$
a_{k}=\sum_{j=1}^{N-2} x_{j}(0) \sin \left(\frac{k j \pi}{N-1}\right)
$$


Of course, when $\alpha$ is zero and the interactions are linear, we are in effect dealing with $N-2$ uncoupled harmonic oscillators (the above normal modes) and there is no thermalization. On the contrary, the sum of the kinetic and potential energy of each of the normal modes is a constant of the motion!

But if $\alpha$ is small but non-zero, FPU expected (on the basis of then generally accepted statistical mechanics arguments) that the energy would gradually shift between modes so as to eventually roughly equalize the total of potential and kinetic energy in each of the $N-2$ normal modes $\xi^{(k)}$. To test this they started the lattice in the fundamental mode $\xi^{(1)}$, with various values of $\alpha$, and integrated Newton's equations numerically for a long time interval, interrupting the evolution from time to time to compute the total of kinetic plus potential energy in each mode. What did they find? Here is a quotation from their report:

Let us say here that the results of our computations show features which were, from the beginning, surprising to us. Instead of a gradual, continuous flow of energy from the first mode to the higher modes, all of the problems showed an entirely different behavior. Starting in one problem with a quadratic force and a pure sine wave as the initial position of the string, we did indeed observe initially a gradual increase of energy in the higher modes as predicted (e.g., by Rayleigh in an infinitesimal analysis). Mode 2 starts increasing first, followed by mode 3 , and so on. Later on, however, this gradual sharing of energy among the successive modes ceases. Instead, it is one or the other mode that predominates. For example, mode 2 decides, as it were, to increase rather rapidly at the cost of the others. At one time it has more energy than all the others put together! Then mode 3 undertakes this rôle. It is only the first few modes which exchange energy among themselves, and they do this in a rather regular fashion. Finally, at a later time, mode 1 comes back to within one percent of its initial value, so that the system seems to be almost periodic.

There is no question that Fermi, Pasta, and Ulam realized they had stumbled onto something big. In his autobiography [Ul], Ulam devotes several pages to a discussion of this collaboration. Here is a little of what he says:

I know that Fermi considered this to be, as he said, "a minor discovery." And when he was invited a year later to give the Gibbs Lecture (a great honorary event at the annual American Mathematical Society meeting), he intended to talk about it. He became ill before the meeting, and his lecture never took place....

The results were truly amazing. There were many attempts to find the reasons for this periodic and regular behavior, which was to be the starting point of what is now a large literature on nonlinear vibrations. Martin Kruskal, a physicist in Princeton, and Norman Zabusky, a mathematician at Bell Labs, wrote papers about it. Later, Peter Lax contributed signally to the theory.

Unfortunately, Fermi died in 1955, even before the paper cited above was published. It was to have been the first in a series of papers, but with Fermi's passing it fell to others to follow up on the striking results of the Fermi-Pasta-Ulam experiments. 
The MANIAC computer, on which FPU carried out their remarkable research, was designed to carry out some computations needed for the design of the first hydrogen bombs, and of course it was a marvel for its day. But it is worth noting that it was very weak by today's standards - not just when compared with current supercomputers, but even when compared with modest desktop machines. At a conference held in 1977 Pasta recalled, "The program was of course punched on cards. A DO loop was executed by the operator feeding in the deck of cards over and over again until the loop was completed!"

2. The Kruskal-Zabusky Experiments. Following the FPU experiments, there were many attempts to explain the surprising quasi-periodicity of solutions of the FPU Lattice Equations. However it was not until ten years later that Martin Kruskal and Norman Zabusky took the crucial steps that led to an eventual understanding of this behavior [ZK].

In fact, they made two significant advances. First they demonstrated that, in a continuum limit, certain solutions of the FPU Lattice Equations could be described in terms of solutions of the so-called Korteweg-de Vries (or KdV) equation. And second, by investigating the initial value problem for the KdV equation numerically on a computer, they discovered that its solutions had remarkable behavior that was related to, but if anything even more surprising and unexpected than the anomalous behavior of the FPU lattice that they had set out to understand.

Finding a good continuum limit for the nonlinear FPU lattice is a lot more sophisticated than one might at first expect after the easy time we had with the linear case. In fact the approach to the limit has to be handled with considerable skill to avoid inconsistent results, and it involves several non-obvious steps.

Let us return to the FPU Lattice Equations

$$
\ddot{x}_{i}=c^{2}\left(\frac{x_{i+1}+x_{i-1}-2 x_{i}}{h^{2}}\right)\left[1+\alpha\left(x_{i+1}-x_{i-1}\right)\right],
$$

and as before let $u(x, t)$ denote the function measuring the displacement at time $t$ of the particle of string with equilibrium position $x$, so if $x=p_{i}$ then, by definition, $x_{i}(t)=u(x, t), x_{i+1}(t)=u(x+h, t)$, and $x_{i-1}(t)=u(x-h, t)$. Of course $\ddot{x}_{i}=$ $u_{t t}(x, t)$ and, as noted earlier, Taylor's Theorem with remainder gives

$$
\begin{aligned}
\frac{x_{i+1}+x_{i-1}-2 x_{i}}{h^{2}} & =\frac{u(x+h, t)+u(x-h, t)-2 u(x, t)}{h^{2}} \\
& =u_{x x}(x, t)+\left(\frac{h^{2}}{12}\right) u_{x x x x}(x, t)+O\left(h^{4}\right) .
\end{aligned}
$$

By a similar computation

$$
\alpha\left(x_{i+1}-x_{i-1}\right)=(2 \alpha h) u_{x}(x, t)+\left(\frac{\alpha h^{3}}{3}\right) u_{x x x}(x, t)+O\left(h^{5}\right),
$$

so substitution in (FPU) gives

$$
\left(\frac{1}{c^{2}}\right) u_{t t}-u_{x x}=(2 \alpha h) u_{x} u_{x x}+\left(\frac{h^{2}}{12}\right) u_{x x x x}+O\left(h^{4}\right) .
$$

As a first attempt to derive a continuum description for the FPU lattice in the nonlinear case, it is tempting to just let $h$ approach zero and assume that $2 \alpha h$ converges to a limit $\epsilon$. This would give the PDE

$$
u_{t t}=c^{2}\left(1+\epsilon u_{x}\right) u_{x x}
$$


as our continuum limit for the FPU Lattice equations and the nonlinear generalization of the wave equation. But this leads to a serious problem. This equation is familiar in applied mathematics - it was studied by Rayleigh in the last century - and it is easy to see from examples that its solutions develop discontinuities (shocks) after a time on the order of $(\epsilon c)^{-1}$, which is considerably shorter than the time scale of the almost periods observed in the Fermi-Pasta-Ulam experiments. It was Zabusky who realized that the correct approach was to retain the term of order $h^{2}$ and study the equation

$$
\left(\frac{1}{c^{2}}\right) u_{t t}-u_{x x}=(2 \alpha h) u_{x} u_{x x}+\left(\frac{h^{2}}{12}\right) u_{x x x x} .
$$

If we differentiate this equation with respect to $x$ and make the substitution $v=u_{x}$, we see that it reduces to the more familiar Boussinesq equation

$$
\left(\frac{1}{c^{2}}\right) v_{t t}=v_{x x}+\alpha h \frac{\partial\left(v^{2}\right)}{\partial x^{2}}+\left(\frac{h^{2}}{12}\right) v_{x x x x} .
$$

(The effect of the fourth order term is to add dispersion to the equation, and this smoothes out incipient shocks before they can develop.)

It is important to realize that, since $h \neq 0,(\mathrm{ZK})$ cannot logically be considered a true continuum limit of the FPU lattice. It should rather be regarded as an asymptotic approximation to the lattice model that works for small lattice spacing $h$ (and hence large $N$ ). Nevertheless, we shall now see how to pass from (ZK) to a true continuum description of the FPU lattice.

The next step is to notice that, with $\alpha$ and $h$ small, solutions of (ZK) should behave qualitatively like solutions of the linear wave equation $u_{t t}=c^{2} u_{x x}$, and increasingly so as $\alpha$ and $h$ tend to zero. Now the general solution of the linear wave equation is of course $u(x, t)=f(x+c t)+g(x-c t)$, i.e., the sum of an arbitrary left moving traveling wave and an arbitrary right moving traveling wave, both moving with speed $c$. Recall that it is customary to simplify the analysis in the linear case by treating each kind of wave separately, and we would like to do the same here. That is, we would like to look for solutions $u(x, t)$ that behave more and more like (say) right moving traveling waves of velocity $c$ - and for longer and longer periods of time - as $\alpha$ and $h$ tend to zero.

It is not difficult to make precise sense out of this requirement. Suppose that $y(\xi, \tau)$ is a smooth function of two real variables such that the map $\tau \mapsto y(\cdot, \tau)$ is uniformly continuous from $\mathbf{R}$ into the bounded functions on $\mathbf{R}$ with the sup normi.e., given $\epsilon>0$ there is a positive $\delta$ such that $\left|\tau-\tau_{0}\right|<\delta$ implies $\left|y(\xi, \tau)-y\left(\xi, \tau_{0}\right)\right|<$ $\epsilon$. Then for $\left|t-t_{0}\right|<T=\delta /(\alpha h c)$ we have $\left|\alpha h c t-\alpha h c t_{0}\right|<\delta$, so $\mid y(x-c t, \alpha h c t)-$ $y\left(x-c t, \alpha h c t_{0}\right) \mid<\epsilon$. In other words, the function $u(x, t)=y(x-c t, \alpha h c t)$ is uniformly approximated by the traveling wave $u^{0}(x, t)=y\left(x-c t, \alpha h c t_{0}\right)$ on the interval $\left|t-t_{0}\right|<T$ (and of course $T \rightarrow \infty$ as $\alpha$ and $h$ tend to zero). To restate this a little more picturesquely, $u(x, t)=y(x-c t, \alpha h c t)$ is approximately a traveling wave whose shape gradually changes in time. Notice that if $y(\xi, \tau)$ is periodic or almost periodic in $\tau$, the gradually changing shape of the approximate traveling wave will also be periodic or almost periodic.

To apply this observation, we define new variables $\xi=x-c t$ and $\tau=(\alpha h) c t$. Then by the chain rule, $\partial^{k} / \partial x^{k}=\partial^{k} / \partial \xi^{k}, \partial / \partial t=-c(\partial / \partial \xi-(\alpha h) \partial / \partial \tau)$, and $\left.\partial^{2} / \partial t^{2}=c^{2}\left(\partial^{2} / \partial \xi^{2}-(2 \alpha h) \partial^{2} / \partial \xi \partial \tau\right)+(\alpha h)^{2} \partial^{2} / \partial \tau^{2}\right)$. 
Thus in these new coordinates the wave operator transforms to:

$$
\frac{1}{c^{2}} \frac{\partial^{2}}{\partial t^{2}}-\frac{\partial^{2}}{\partial x^{2}}=-2 \alpha h \frac{\partial^{2}}{\partial \xi \partial \tau}+(\alpha h)^{2} \frac{\partial^{2}}{\partial \tau^{2}},
$$

so substituting $u(x, t)=y(\xi, \tau)$ in (ZK) (and dividing by $-2 \alpha h$ ) gives:

$$
y_{\xi \tau}-\left(\frac{\alpha h}{2}\right) y_{\tau \tau}=-y_{\xi} y_{\xi \xi}-\left(\frac{h}{24 \alpha}\right) y_{\xi \xi \xi \xi},
$$

and, at last, we are prepared to pass to the continuum limit. We assume that $\alpha$ and $h$ tend to zero at the same rate, i.e., that as $h$ tends to zero, the quotient $h / \alpha$ tends to a positive limit, and we define $\delta=\lim _{h \rightarrow 0} \sqrt{h /(24 \alpha)}$. Then $\alpha h=O\left(h^{2}\right)$, so letting $h$ approach zero gives $y_{\xi \tau}+y_{\xi} y_{\xi \xi}+\delta^{2} y_{\xi \xi \xi \xi}=0$. Finally, making the substitution $v=y_{\xi}$ we arrive at the KdV equation:

$$
v_{\tau}+v v_{\xi}+\delta^{2} v_{\xi \xi \xi}=0 .
$$

Remark. Note that if we re-scale the independent variables by $\tau \rightarrow \beta \tau$ and $\xi \rightarrow \gamma \xi$, then the KdV equation becomes:

$$
v_{\tau}+\left(\frac{\beta}{\gamma}\right) v v_{\xi}+\left(\frac{\beta}{\gamma^{3}}\right) \delta^{2} v_{\xi \xi \xi}=0
$$

so by appropriate choice of $\beta$ and $\gamma$ we can obtain any equation of the form $v_{\tau}+$ $\lambda v v_{\xi}+\mu v_{\xi \xi \xi}=0$, and any such equation is referred to as "the KdV equation". A commonly used choice that is convenient for many purposes is $v_{\tau}+6 v v_{\xi}+v_{\xi \xi \xi}=0$, although the form $v_{\tau}-6 v v_{\xi}+v_{\xi \xi \xi}=0$ (obtained by replacing $v$ by $-v$ ) is equally common. We will use both these forms.

Let us recapitulate the relationship between the FPU Lattice and the KdV equation. Given a solution $x_{i}(t)$ of the FPU Lattice, we get a function $u(x, t)$ by interpolation-i.e., $u(i h, t)=x_{i}(t), i=0, \ldots, N$. For small lattice spacing $h$ and nonlinearity parameter $\alpha$ there will be solutions $x_{i}(t)$ so that the corresponding $u(x, t)$ will be an approximate right moving traveling wave with slowly varying shape, i.e., it will be of the form $u(x, t)=y(x-c t, \alpha h c t)$ for some smooth function $y(\xi, \tau)$, and the function $v(\xi, \tau)=y_{\xi}(\xi, \tau)$ will satisfy the $\mathrm{KdV}$ equation $v_{\tau}+v v_{\xi}+\delta^{2} v_{\xi \xi \xi}=0$, where $\delta^{2}=h /(24 \alpha)$.

Having found this relationship between the FPU Lattice and the KdV equation, Kruskal and Zabusky made some numerical experiments, solving the KdV initial value problem for various initial data. Before discussing the remarkable results that came out of these experiments, it will be helpful to recall some of the early history of this equation.

3. A First Look at KdV. Korteweg and de Vries derived their equation in 1895 to settle a debate that had been going on since 1844, when the naturalist and naval architect John Scott Russell, in an oft-quoted paper [Ru], reported an experience a decade earlier in which he followed the bow wave of a barge that had suddenly stopped in a canal. This "solitary wave", some thirty feet long and a foot high, moved along the channel at about eight miles per hour, maintaining its shape and speed for over a mile as Russell raced after it on horseback. Russell became fascinated with this phenomenon and made extensive further experiments with such waves in a wave tank of his own devising, eventually deriving a (correct) formula for their speed as a function of height. The mathematicians Airy and Stokes made calculations which appeared to show that any such wave would be unstable and not persist for as long as Russell claimed. However, later work by Boussinesq (1872), 
Rayleigh (1876) and finally the Korteweg-de Vries paper in $1895[\mathrm{KdV}]$ pointed out errors in the analysis of Airy and Stokes and vindicated Russell's conclusions.

The KdV equation is now accepted as controlling the dynamics of waves moving to the right in a shallow channel. Of course, Korteweg and de Vries did the obvious and looked for traveling-wave solutions for their equation by making the Ansatz $v(x, t)=f(x-c t)$. When this is substituted in the standard form of the $\mathrm{KdV}$ equation, it gives $-c f^{\prime}+6 f f^{\prime}+f^{\prime \prime \prime}=0$. If we add the boundary conditions that $f$ should vanish at infinity, then a fairly routine analysis leads to the one-parameter family of traveling-wave solutions $v(x, t)=2 a^{2} \operatorname{sech}^{2}\left(a\left(x-4 a^{2} t\right)\right)$, now referred to as the one-soliton solutions of $\mathrm{KdV}$. (These are of course the solitary waves of Russell.) Note that the amplitude $2 a^{2}$ is exactly half the speed $4 a^{2}$, so that taller waves move faster than their shorter brethren.

Now, back to Zabusky and Kruskal. For numerical reasons, they chose to deal with the case of periodic boundary conditions - in effect studying the KdV equation $u_{t}+u u_{x}+\delta^{2} u_{x x x}=0$ (which they label (1) ) on the circle instead of on the line. For their published report, they chose $\delta=0.022$ and used the initial condition $u(x, 0)=\cos (\pi x)$. Here is an extract from their report (containing the first use of the term "soliton") in which they describe their observations:

(I) Initially the first two terms of Eq. (1) dominate and the classical overtaking phenomenon occurs; that is $u$ steepens in regions where it has negative slope. (II) Second, after $u$ has steepened sufficiently, the third term becomes important and serves to prevent the formation of a discontinuity. Instead, oscillations of small wavelength (of order $\delta$ ) develop on the left of the front. The amplitudes of the oscillations grow, and finally each oscillation achieves an almost steady amplitude (that increases linearly from left to right) and has the shape of an individual solitary-wave of (1). (III) Finally, each "solitary wave pulse" or soliton begins to move uniformly at a rate (relative to the background value of $u$ from which the pulse rises) which is linearly proportional to its amplitude. Thus, the solitons spread apart. Because of the periodicity, two or more solitons eventually overlap spatially and interact nonlinearly. Shortly after the interaction they reappear virtually unaffected in size or shape. In other words, solitons "pass through" one another without losing their identity. Here we have a nonlinear physical process in which interacting localized pulses do not scatter irreversibly.

(If you are not sure what Zabusky and Kruskal mean here by "the classical overtaking phenomenon", it will be explained in the next section.)

Zabusky and Kruskal go on to describe a second interesting observation, a recurrence property of the solitons that goes a long way towards accounting for the surprising recurrence observed in the FPU Lattice. Let us explain again, but in somewhat different terms, the reason why the recurrence in the FPU Lattice is so surprising. The lattice is made up of a great many identical oscillators. Initially the relative phases of these oscillators are highly correlated by the imposed cosine initial condition. If the interactions are linear $(\alpha=0)$, then the oscillators are harmonic and their relative phases remain constant. But, when $\alpha$ is positive, the anharmonic forces between the oscillators cause their phases to start drifting relative to each other in an apparently uncorrelated manner. The expected time before the phases of all of the oscillators will be simultaneously close to their initial phases 
is enormous, and increases rapidly with the total number $N$. But, from the point of view of the KdV solitons, an entirely different picture appears. As mentioned in the above paragraph, if $\delta$ is put equal to zero in the $\mathrm{KdV}$ equation, it reduces to the so-called inviscid Burgers' Equation, which exhibits steepening and breaking of a negatively sloped wave front in a finite time $T_{B}$. (For the above initial conditions, the breaking time, $T_{B}$, can be computed theoretically to be $1 / \pi$.) However, when $\delta>0$, just before breaking would occur, a small number of solitons emerge (eight in the case of the above initial wave shape, $\cos (\pi x)$ ) and this number depends only on the initial wave shape, not on the number of oscillators. The expected time for their respective centers of gravity to all eventually "focus" at approximately the same point of the circle is of course much smaller than the expected time for the much larger number of oscillators to all return approximately to their original phases. In fact, the recurrence time $T_{R}$ for the solitons turns out to be approximately equal to $30.4 T_{B}$, and at this time the wave shape $u\left(x, T_{R}\right)$ is uniformly very close to the initial wave form $u(x, 0)=\cos (\pi x)$. There is a second (somewhat weaker) focusing at time $t=2 T_{R}$, etc. (Note that these times are measured in units of the "slow time", $\tau$, at which the shape of the FPU traveling wave evolves, not in the "fast time", $t$, at which the traveling wave moves.) In effect, the KdV solitons are providing a hidden correlation between the relative phases of the FPU oscillators!

Notice that, as Zabusky and Kruskal emphasize, it is the persistence or shape conservation of the solitons that provides the explanation of recurrence. If the shapes of the solitons were not preserved when they interacted, there would be no way for them to all get back together and approximately reconstitute the initial condition at some later time. Here in their own words is how they bring in solitons to account for the fact that thermalization was not observed in the FPU experiment:

Furthermore, because the solitons are remarkably stable entities, preserving their identities throughout numerous interactions, one would expect this system to exhibit thermalization (complete energy sharing among the corresponding linear normal modes) only after extremely long times, if ever.

But this explanation, elegant as it may be, only pushes the basic question back a step. A full understanding of FPU recurrence requires that we comprehend the reasons behind the remarkable new phenomenon of solitonic behavior, and in particular why solitons preserve their shape. In fact, it was quickly recognized that the soliton was itself a vital new feature of nonlinear dynamics, so that understanding it better and discovering other nonlinear wave equations that had soliton solutions became a primary focus for research in both pure and applied mathematics. The mystery of the FPU Lattice recurrence soon came to be regarded as an important but fortuitous spark that ignited this larger effort.

The next few short sections explain some elementary but important facts about one-dimensional wave equations. If you know about shock development, and how dispersion smooths shocks, you can skip these sections without loss of continuity.

4. "Steepening" and "Breaking". Several times already we have referred to the phenomenon of "steepening and breaking of negatively sloped wave-fronts" for certain wave equations. If you have never seen this explained, it probably sounds suggestive but also a little mysterious. In fact something very simple is going on that we will now explain. 
Let us start with the most elementary of all one-dimensional wave equations, the linear advection equation (or forward wave equation), $u_{t}+c u_{x}=0$. If we think of the graph of $x \mapsto u(x, t)$ as representing the profile of a wave at time $t$, then this equation describes a special evolutionary behavior of the wave profile in time. In fact, if $u_{0}(x)=u(x, 0)$ is the "initial" shape of the wave, then the unique solution of the equation with this initial condition is the so-called "traveling wave" $u(x, t)=u_{0}(x-c t)$, i.e., just the initial wave profile translating rigidly to the right at a uniform velocity $c$. In other words, we can construct the wave profile at time $t$ by translating each point on the graph of $u_{0}(x)$ horizontally by an amount $c t$. As we shall now see, this has a remarkable generalization.

We shall be interested in the non-viscous Burgers' equation, $u_{t}+u u_{x}=0$, but it is just as easy to treat the more general equation $u_{t}+f(u) u_{x}=0$, where $f: \mathbf{R} \rightarrow \mathbf{R}$ is some smooth function. Let me call this simply the nonlinear advection equation or NLA.

Proposition. Let $u(x, t)$ be a smooth solution of the nonlinear advection equation $u_{t}+f(u) u_{x}=0$ for $x \in \mathbf{R}$ and $t \in\left[0, t_{0}\right]$, and with initial condition $u_{0}(x)=u(x, 0)$. Then for $t<t_{0}$ the graph of $x \mapsto u(x, t)$ can be constructed from the graph of $u_{0}$ by translating each point $\left(x, u_{0}(x)\right)$ horizontally by an amount $f\left(u_{0}(x)\right) t$.

Proof. The proof is by the "method of characteristics", i.e., we look for curves $(x(s), t(s))$ along which $u(x, t)$ must be a constant (say $c$ ), because $u$ satisfies NLA. If we differentiate $u(x(s), t(s))=c$ with respect to $s$, then the chain rule gives $u_{x}(x(s), t(s)) x^{\prime}(s)+u_{t}(x(s), t(s)) t^{\prime}(s)=0$, and hence $d x / d t=x^{\prime}(s) / t^{\prime}(s)=$ $-u_{t}(x(s), t(s)) / u_{x}(x(s), t(s))$, and now substitution from NLA gives:

$$
d x / d t=f(u(x(s), t(s)))=f(c),
$$

so the characteristic curves are straight lines, whose slope is $f(c)$, where $c$ is the constant value the solution $u$ has along that line. In particular, if we take the straight line with slope $f\left(u_{0}(x)\right)$ starting from the point $(x, 0)$, then $u(x, t)$ will have the constant value $u_{0}(x)$ along this line, a fact that is equivalent to the conclusion of the Proposition.

It is now easy to explain steepening and breaking. We assume that the function $f$ is monotonically increasing and that $u_{0}(x)$ has negative slope (i.e., is strictly decreasing) on some interval $I$. If we follow the part of the wave profile that is initially over the interval $I$, we see from the Proposition that the higher part (to the left) will move faster than the lower part (to the right), and so gradually overtake it. The result is that the wave "bunches up" and its slope increases - this is steepening - and eventually there will be a first time $T_{B}$ when the graph has a vertical tangent - this is breaking. Clearly the solution cannot be continued past $t=T_{B}$, since for $t>T_{B}$ the Proposition would give a multi-valued graph for $u(x, t)$. It is an easy exercise to show that the breaking time $T_{B}$ is given by $\left|\min \left(u_{0}^{\prime}(x)\right)\right|^{-1}$.

This explains the first part of the above quotation from Zabusky and Kruskal, namely, "Initially the first two terms of Eq. (1) dominate and the classical overtaking phenomenon occurs; that is $u$ steepens in regions where it has negative slope." But what about their next comment: "Second, after $u$ has steepened sufficiently, the third term becomes important and serves to prevent the formation of a discontinuity"? To explain this we have to take up the matter of dispersion. 
5. Dispersion. Let us next consider linear wave equations of the form $u_{t}+$ $P\left(\frac{\partial}{\partial x}\right) u=0$, where $P$ is a polynomial. Recall that a solution $u(x, t)$ of the form $e^{i(k x-\omega t)}$ is called a plane-wave solution; $k$ is called the wave number (waves per unit length) and $\omega$ the (angular) frequency. Rewriting this in the form $e^{i k(x-(\omega / k) t)}$, we recognize that this is a traveling wave of velocity $\frac{\omega}{k}$. If we substitute this $u(x, t)$ into our wave equation, we get a formula determining a unique frequency $\omega(k)$ associated to any wave number $k$, which we can write in the form $\frac{\omega(k)}{k}=\frac{1}{i k} P(i k)$. This is called the "dispersion relation" for this wave equation. Note that it expresses the velocity for the plane-wave solution with wave number $k$. For example, $P\left(\frac{\partial}{\partial x}\right)=c \frac{\partial}{\partial x}$ gives the linear advection equation $u_{t}+c u_{x}=0$, which has the dispersion relation $\frac{\omega(k)}{k}=c$, showing of course that all plane-wave solutions travel at the same velocity $c$, and we say that we have trivial dispersion in this case. On the other hand, if we take $P\left(\frac{\partial}{\partial x}\right)=\left(\frac{\partial}{\partial x}\right)^{3}$, then our wave equation is $u_{t}+u_{x x x}=0$, which is the $\mathrm{KdV}$ equation without its nonlinear term, and we have the non-trivial dispersion relation $\frac{\omega(k)}{k}=-k^{2}$. In this case, plane waves of large wave-number (and hence high frequency) are traveling much faster than low-frequency waves. The effect of this is to "broaden a wave-packet". That is, suppose our initial condition is $u_{0}(x)$. We can use the Fourier Transform to write $u_{0}$ in the form $u_{0}(x)=\int \hat{u}_{0}(k) e^{i k x} d k$, and then, by superposition, the solution to our wave equation will be $u(x, t)=$ $\int \hat{u}_{0}(k) e^{i k(x-(\omega(k) / k) t)} d k$. Suppose for example our initial wave form is a highly peaked Gaussian. Then in the case of the linear advection equation all the Fourier modes travel together at the same speed and the Gaussian lump remains highly peaked over time. On the other hand, for the linearized KdV equation the various Fourier modes all travel at different velocities, so after a short time they start cancelling each other by destructive interference, and the originally sharp Gaussian quickly broadens. This is what Zabusky and Kruskal are referring to when they say that "... the third term becomes important and serves to prevent the formation of a discontinuity." Just before breaking or shock-formation, the broadening effects of dispersion start to cancel the peaking effects of steepening. Indeed, careful analysis shows that in some sense, what gives KdV solitons their special properties of stability and longevity is a fine balance between the yin effects of dispersion and the yang effects of steepening.

6. Split-Stepping $\mathbf{K d V}$. There is an interesting question that is suggested by our analysis in the last two sections. In the KdV equation, $u_{t}=-6 u u_{x}-u_{x x x}$, if we drop the nonlinear term, we have a constant coefficient linear PDE whose initial value problem can be solved explicitly by the Fourier Transform. On the other hand, if we ignore the linear third-order term, then we are left with the inviscid Burgers' equation, whose initial value problem can be solved numerically by a variety of methods. (It can also be solved in implicit form analytically, for short times, by the method of characteristics,

$$
u=u_{o}(x-6 u t),
$$

but the solution is not conveniently represented on a fixed numerical grid.) Can we somehow combine the methods for solving each of the two parts into an efficient numerical method for solving the full KdV initial value problem?

In fact we can, and indeed there is a very general technique that applies to such situations. In the pure mathematics community it is usually referred to as the Trotter Product Formula, while in the applied mathematics and numerical analysis 
communities it is called split-stepping. Let me state it in the context of ordinary differential equations. Suppose that $Y$ and $Z$ are two smooth vector fields on $\mathbf{R}^{n}$, and we know how to solve each of the differential equations $d x / d t=Y(x)$ and $d x / d t=Z(x)$, meaning that we know both of the flows $\phi_{t}$ and $\psi_{t}$ on $\mathbf{R}^{n}$ generated by $X$ and $Y$ respectively. The Trotter Product Formula is a method for constructing the flow $\theta_{t}$ generated by $Y+Z$ out of $\phi$ and $\psi$; namely, letting $\Delta t=\frac{t}{n}, \theta_{t}=\lim _{n \rightarrow \infty}\left(\phi_{\Delta t} \psi_{\Delta t}\right)^{n}$. The intuition behind the formula is simple. Think of approximating the solution of $d x / d t=Y(x)+Z(x)$ by Euler's Method. If we are currently at a point $p_{0}$, to propagate one more time step $\Delta t$ we go to the point $p_{0}+\Delta t\left(Y\left(p_{0}\right)+Z\left(p_{0}\right)\right)$. Using the split-step approach on the other hand, we first take an Euler step in the $Y\left(p_{0}\right)$ direction, going to $p_{1}=p_{0}+\Delta t Y\left(p_{0}\right)$, then take a second Euler step, but now from $p_{1}$ and in the $Z\left(p_{1}\right)$ direction, going to $p_{2}=p_{1}+\Delta t Z\left(p_{1}\right)$. If $Y$ and $Z$ are constant vector fields, then this gives exactly the same final result as the simple full Euler step with $Y+Z$, while for continuous $Y$ and $Z$ and small time step $\Delta t$ it is a good enough approximation that the above limit is valid.

The situation is more delicate for flows on infinite dimensional manifolds. Nevertheless it was shown by F. Tappert in $[\mathrm{Ta}]$ that the Cauchy Problem for KdV can be solved numerically by using split-stepping to combine solution methods for $u_{t}=-6 u u_{x}$ and $u_{t}=-u_{x x x}$. In addition to providing a perspective on an evolution equation's relation to its component parts, split-stepping allows one to modify a code from solving $\mathrm{KdV}$ to the Kuramoto-Sivashinsky equation $\left(u_{t}+u u_{x}=\right.$ $\left.-u_{x x}-u_{x x x x}\right)$, or study the joint zero-diffusion-dispersion limits KdV-Burgers' equation $\left(u_{t}+6 u u_{x}=\nu u_{x x}+\epsilon u_{x x x x}\right)$, by merely changing one line of code in the Fourier module.

Tappert uses an interesting variant, known as Strang splitting, which was first suggested in [St] to solve multi-dimensional hyperbolic problems by split-stepping one-dimensional problems. The advantage of splitting comes from the greatly reduced effort required to solve the smaller bandwidth linear systems which arise when implicit schemes are necessary to maintain stability. In addition, Strang demonstrated that second-order accuracy of the component methods need not be compromised by the asymmetry of the splitting, as long as the pattern $\phi_{\frac{\Delta t}{2}} \psi_{\frac{\Delta t}{2}} \psi_{\frac{\Delta t}{2}} \phi_{\frac{\Delta t}{2}}$ is used, to account for possible non-commutativity of $Y$ and $Z$. (This may be seen by multiplying the respective exponential series.) No higher order analogue of Strang splitting is available. Serendipitously, when output is not required, several steps of Strang splitting require only marginal additional effort: $\left(\phi_{\frac{\Delta t}{2}} \psi_{\frac{\Delta t}{2}} \psi_{\frac{\Delta t}{2}} \phi_{\frac{\Delta t}{2}}\right)^{n}=$ $\left(\phi_{\frac{\Delta t}{2}} \psi_{\Delta t}\left(\phi_{\Delta t} \psi_{\Delta t}\right)^{n-1} \phi_{\frac{\Delta t}{2}}\right.$.

7. A Symplectic Structure for KdV. The FPU Lattice is a classical finite dimensional mechanical system, and as such it has a natural Hamiltonian formulation. However its relation to $\mathrm{KdV}$ is rather complex - and $\mathrm{KdV}$ is a PDE rather than a finite dimensional system of ODE - so it is not clear that it too can be viewed as a Hamiltonian system. We shall now see how this can be done in a simple and natural way. Moreover, when interpreted as the infinite dimensional analogue of a Hamiltonian system, KdV turns out to have a key property one would expect from any generalization to infinite dimensions of the concept of complete integrability in the Liouville sense, namely the existence of infinitely many functionally independent constants of the motion that are in involution. (Later, in discussing the inverse scattering method, we will indicate how complete integrability was proved 
in a more precise sense by Fadeev and Zakharov $[\mathrm{ZF}]$; they demonstrated that the "scattering data" for the KdV equation obey the characteristic Poisson bracket relations for the action-angle variables of a completely integrable system.)

In 1971, Gardiner and Zakharov independently showed how to interpret KdV as a Hamiltonian system, starting from a Poisson bracket approach, and from this beginning Poisson brackets have played a significantly more important rôle in the infinite dimensional theory of Hamiltonian systems than they did in the more classical finite dimensional theory, and in recent years this has led to a whole theory of so-called Poisson manifolds and Poisson Lie groups. However, we will start with the more classical approach to Hamiltonian systems, defining a symplectic structure for KdV first, and then obtain the Poisson bracket structure as a derived concept (cf. Abraham and Marsden $[\mathrm{AbM}]$ ). Thus, we will first exhibit a symplectic structure $\Omega$ for the phase space $P$ of the KdV equation and a Hamiltonian function, $H: P \rightarrow \mathbf{R}$, such that the KdV equation takes the form $\dot{u}=\left(\nabla_{s} H\right)_{u}$.

For simplicity, we shall take as our phase space $P$ the Schwartz space, $\mathcal{S}(\mathbf{R})$, of rapidly decreasing functions $u: \mathbf{R} \rightarrow \mathbf{R}$, although a much larger space would be possible. (In $[\mathrm{BS}]$ it is proved that $\mathrm{KdV}$ defines a global flow on the Sobolev space $H^{4}(\mathbf{R})$ of functions $u: \mathbf{R} \rightarrow \mathbf{R}$ with derivatives of order up to 4 in $L^{2}$, and it is not hard to see that $P$ is an invariant subspace of this flow. See also [Ka1], [Ka2].) For $u, v$ in $P$ we will denote their $L^{2}$ inner product $\int_{-\infty}^{\infty} u(x) v(x) d x$ by $\langle u, v\rangle$ and we define

$$
\Omega(u, v)=\frac{1}{2} \int_{-\infty}^{\infty}\left(v(x) \int u(x)-u(x) \int v(x)\right) d x,
$$

where $f u(x)=\int_{-\infty}^{x} u(y) d y$ denotes the indefinite integral of $u$. (For the periodic $\mathrm{KdV}$ equation we take $P$ to be all smooth periodic functions of period $2 \pi$ and replace the $\int_{-\infty}^{\infty}$ by $\int_{0}^{2 \pi}$.)

We denote by $\partial$ the derivative operator, $u \mapsto u^{\prime}$, so $\partial \int u=u$, and $\int_{-\infty}^{\infty} \partial u=0$ for functions $u$ that vanish at infinity. We will also write $u_{(k)}$ for $\partial^{k} u$, but for small $k$ we shall also use $u=u_{(0)}, u_{x}=u_{(1)}, u_{x x}=u_{(2)}$, etc.

There is a simple but important relation connecting $\Omega, \partial$, and the $L^{2}$ inner product, namely:

$$
\Omega(\partial u, v)=\langle u, v\rangle .
$$

This is an immediate consequence of three obvious identities: $\partial(u f v)=(\partial u) f v+u v$, $\int_{-\infty}^{\infty} \partial(u \jmath v)=0$, and $\Omega(\partial u, v)=(1 / 2) \int_{-\infty}^{\infty}\left(v u-(\partial u) \int v\right)$.

One important consequence of this is the weak non-degeneracy of $\Omega$. For, if $i_{v} \Omega$ is zero, then in particular $\langle u, v\rangle=\Omega(\partial u, v)=-\Omega(v, \partial u)=-\left(i_{v} \Omega\right)(\partial u)=0$ for all $u$, so $v=0$.

$\Omega$ is clearly a skew-bilinear form on $P$. Since $P$ is a vector space, we can as usual identify $P$ with its tangent space at every point, and then $\Omega$ becomes a "constant" 2 -form on $P$. Since it is constant, of course $d \Omega=0$. (Below we will exhibit an explicit 1-form $\omega$ on $P$ such that $d \omega=\Omega$.) Thus $\Omega$ is a symplectic form for $P$, and henceforth we will consider $P$ to be a symplectic manifold.

A second consequence of $\Omega(\partial u, v)=\langle u, v\rangle$ is that if $F: P \rightarrow \mathbf{R}$ is a smooth function (or "functional") on $P$ that has a gradient $\nabla F$ with respect to the flat Riemannian structure on $P$ defined by the $L^{2}$ inner product, then the symplectic gradient of $F$ also exists and is given by $\left(\nabla_{s} F\right)_{u}=\partial\left((\nabla F)_{u}\right)$. Recall that $d F$, the 
differential of $F$, is the 1 -form on $P$ defined by

$$
d F_{u}(v)=\left.\frac{d}{d \epsilon}\right|_{\epsilon=0} F(u+\epsilon v),
$$

and the gradient of $F$ is the vector field dual to $d F$ with respect to the $L^{2}$ inner product (if such a vector field indeed exists), i.e., it is characterized by $(d F)_{u}(v)=$ $\left\langle(\nabla F)_{u}, v\right\rangle$. Since $\left\langle(\nabla F)_{u}, v\right\rangle=\Omega\left(\left(\partial(\nabla F)_{u}\right), v\right)$, it then follows that $\left(\nabla_{s} F\right)_{u}$ also exists and equals $\partial\left((\nabla F)_{u}\right)$.

We shall only consider functions $F: P \rightarrow \mathbf{R}$ of the type normally considered in the Calculus of Variations, i.e., of the form:

$$
F(u)=\int_{-\infty}^{\infty} \tilde{F}\left(u, u_{x}, u_{x x}, \ldots\right) d x
$$

where $\tilde{F}: \mathbf{R}^{k+1} \rightarrow \mathbf{R}$ is a polynomial function without a constant term. Then the usual integration by parts argument of the Calculus of Variations shows that such an $F$ has a gradient, given by:

$$
(\nabla F)_{u}=\frac{\partial \tilde{F}}{\partial u}-\partial\left(\frac{\partial \tilde{F}}{\partial u_{x}}\right)+\partial^{2}\left(\frac{\partial \tilde{F}}{\partial u_{x x}}\right)-\ldots
$$

Remark. The above formula is written using the standard but somewhat illogical conventions of the Calculus of Variations and needs a little interpretation. $\tilde{F}$ is a function of variables $y=\left(y_{0}, y_{1}, y_{2}, \ldots y_{k}\right)$, and for example $\partial \tilde{F} / \partial u_{x x}$ really means the function on $\mathbf{R}$ whose value at $x$ is $\partial \tilde{F} / \partial y_{2}$ evaluated at $y=$ $\left(u_{(0)}(x), u_{(1)}(x), u_{(2)}(x), \ldots u_{(k)}(x)\right)$.

From what we saw above, the symplectic gradient of such an $F$ exists and is given by:

$$
\left(\nabla_{s} F\right)_{u}=\partial\left(\frac{\partial \tilde{F}}{\partial u}\right)-\partial^{2}\left(\frac{\partial \tilde{F}}{\partial u_{x}}\right)+\partial^{3}\left(\frac{\partial \tilde{F}}{\partial u_{x x}}\right)-\ldots
$$

Thus every such $F$ is a Hamiltonian function on $P$, defining the Hamiltonian flow $\dot{u}=\left(\nabla_{s} F\right)_{u}$, where $u(t)$ denotes a smooth curve in $P$. If instead of $u(t)(x)$ we write $u(x, t)$, this symbolic ODE in the manifold $P$ becomes the PDE:

$$
u_{t}=\partial\left(\frac{\partial \tilde{F}}{\partial u}\right)-\partial^{2}\left(\frac{\partial \tilde{F}}{\partial u_{x}}\right)+\partial^{3}\left(\frac{\partial \tilde{F}}{\partial u_{x x}}\right)-\ldots
$$

In particular if we take $\tilde{F}\left(u, u_{x}\right)=-u^{3}+u_{x}^{2} / 2$, then we get the KdV equation in standard form: $u_{t}=\partial\left(-3 u^{2}\right)-\partial^{2}\left(u_{x}\right)=-6 u u_{x}-u_{x x x}$.

Remark. The formula defining $\Omega$ can be motivated as follows. Define linear functionals $p_{x}$ and $q_{x}$ on $P$ by $q_{x}(u)=u(x)$ and $p_{x}(u)=\int u(x)$. (Think of these as providing "continuous coordinates" for $P$.) These give rise to differential 1 -forms $d p_{x}$ and $d q_{x}$ on $P$. Of course, since $p_{x}$ and $q_{x}$ are linear, at every point $u$ of $P$, we have $d p_{x}=p_{x}$ and $d q_{x}=q_{x}$. Then $\Omega$ can now be written in the suggestive form $\Omega=\sum_{x} d p_{x} \wedge d q_{x}$, where $\sum_{x}$ is shorthand for $\int_{-\infty}^{\infty}$. This suggests that we define a 1 -form $\omega$ on $P$ by $\omega=\sum_{x} p_{x} d q_{x}$, i.e., $\omega_{w}(u)=\int_{-\infty}^{\infty} \int w(x) u(x) d x$. Consider this as a function $f(w)$ on $P$ and let us compute its directional derivative at $w$ in the direction $v,(v f)(w)=\left.\frac{d}{d \epsilon}\right|_{\epsilon=0} f(w+\epsilon v)$. We clearly get $v(\omega(u))=\int_{-\infty}^{\infty} f v(x) u(x) d x$. Since $u$ and $v$ are constant vector fields, their bracket $[u, v]$ is zero, and we calculate $d \omega(u, v)=v(\omega(u))-u(\omega(v))=\Omega(u, v)$, as expected. 
We now again specialize to the phase space $P$ for the KdV equation, namely the Schwartz space $\mathcal{S}(\mathbf{R})$ with its $L^{2}$ inner product $\langle u, v\rangle$ and symplectic form $\Omega(u, v)$, related by $\Omega(\partial u, v)=\langle u, v\rangle$. Then, since $\nabla_{s} F=\partial(\nabla F)$, we obtain the formula

$$
\left\{F_{1}, F_{2}\right\}=\Omega\left(\nabla_{s} F_{2}, \nabla_{s} F_{1}\right)=\Omega\left(\partial \nabla F_{2}, \partial \nabla F_{1}\right)=\left\langle\nabla F_{2}, \partial\left(\nabla F_{1}\right)\right\rangle
$$

for Poisson brackets in terms of the Riemannian structure for $P$, and in particular we see that $F_{1}$ and $F_{2}$ are in involution if and only if the two vector fields $\nabla F_{1}$ and $\partial \nabla F_{2}$ on $P$ are everywhere orthogonal.

\section{The Inverse Scattering Method}

In 1967, in what would prove to be one of the most cited mathematical papers in history, [GGKM], Clifford Gardner, John Greene, Martin Kruskal, and Robert Miura introduced an ingenious method, called the Inverse Scattering Transform (IST), for solving the KdV equation. In the years that followed, the IST changed applied mathematics like no other tool since the Fourier Transform (to which it is closely related), and it soon became clear that it was the key to understanding the remarkable properties of soliton equations.

Before starting to explain the IST, we recall the basic philosophy of using "transforms" to solve ODE. Suppose we are interested in some evolution equation $\dot{x}=X(x)$ on a smooth manifold $M$. That is, $X$ is a smooth vector field on $M$ that generates a flow $\phi_{t}$ on $M$. Usually our goal is to understand the dynamical properties of this flow - and perhaps get an explicit "formula" for $\phi_{t}(x)$, at least for some initial conditions $x$. A transform is a diffeomorphism $T$ of $M$ onto some other manifold $N$, mapping the vector $X$ onto a vector field $Y=D T(X)$ on $N$. If $\psi_{t}$ is the flow generated by $Y$, then clearly $T\left(\phi_{t}(x)\right)=\psi_{t}(T x)$, and it follows that if we understand $\psi_{t}$ well, and moreover have explicit methods for computing $T(x)$ and $T^{-1}(y)$, then we in effect also know all about $\phi_{t}$.

It is important to realize that there is usually more at stake than just finding particular solutions of the original initial value problem. Essential structural features of the flow that are hidden from view in the original form of the evolution equation may become manifest when viewed in the transform space $N$.

For example, consider the case of a linear evolution equation $\dot{x}=X(x)$ on some vector space $M$. We can formally "solve" such an equation in the form $x(t)=\exp (t X) x(0)$. However, explicit evaluation of the linear operator $\exp (t X)$ is not generally feasible, nor does the formula provide much insight into the structure of the flow. But suppose we can find a linear diffeomorphism $T: M \rightarrow N$ so that the linear operator $Y=T X T^{-1}$ is diagonal in some "basis" (discrete or continuous) $\left\{w_{\alpha}\right\}$ for $N$, say $Y w_{\alpha}=\lambda_{\alpha} w_{\alpha}$. Then $\exp (t Y) w_{\alpha}=e^{\lambda_{\alpha} t} w_{\alpha}$; hence if $y(0)=\sum_{\alpha} \hat{y}_{\alpha} w_{\alpha}$, then the solution to the initial value problem $\dot{y}=Y(y)$ with initial value $y(0)$ is $y(t)=\sum_{\alpha}\left(e^{\lambda_{\alpha} t} \hat{y}_{\alpha}\right) w_{\alpha}$. Not only do we have an explicit formula for $\psi_{t}$, but we see the important structural fact that the flow is just a direct sum (or integral) of uncoupled one-dimensional flows, something not obvious when viewing the original flow.

This is precisely why the Fourier transform is such a powerful tool for analyzing constant coefficient linear PDE-it simultaneously diagonalizes all such operators! Since the Fourier transform is an excellent model for understanding the more complex IST, let us quickly review it in our current context. It will be convenient to complexify $P$ temporarily, i.e., regard our phase space as the complex vector space of complex-valued Schwartz functions on $\mathbf{R}$. Then the Fourier 
Transform, $v \mapsto w=\mathcal{F}(v)$, is a linear diffeomorphism of $P$ with itself, defined by $w(\alpha)=\frac{1}{\sqrt{2 \pi}} \int_{-\infty}^{\infty} v(x) e^{-i \alpha x} d x$, and the Inverse Fourier Transform, $w \mapsto v=\mathcal{I F}(w)$ is given by $v(x)=\frac{1}{\sqrt{2 \pi}} \int_{-\infty}^{\infty} w(\alpha) e^{i \alpha x} d \alpha$.

Given any $n+1$-tuple of real numbers $a=\left(a_{0}, \ldots a_{n}\right)$, we let $F_{a}(y)$ denote the polynomial $a_{0} y+a_{1} y^{3}+\ldots+a_{n} y^{2 n+1}$, and $F_{a}(\partial)$ the constant coefficient linear differential operator $a_{0} \partial+a_{1} \partial^{3}+\ldots+a_{n} \partial^{2 n+1}$. Note that $F_{a}(\partial)$ is a vector field on $P$. In fact, if we put $\tilde{H}_{a}\left(v_{(0)}, \ldots, v_{(n)}\right)=\frac{1}{2} \sum_{j=0}^{n} a_{j}\left(v_{(j)}\right)^{2}$, and define the corresponding functional $H_{a}(v)=\int_{-\infty}^{\infty} \tilde{H}_{a}\left(v_{(0)}, \ldots, v_{(n)}\right) d x$, then clearly $F_{a}(\partial)=$ $\nabla_{s} H_{a}$. It is trivial that if $b=\left(b_{0}, \ldots b_{m}\right)$ is some other $m+1$-tuple of real numbers, then $\left[F_{a}(\partial), F_{b}(\partial)\right]=0$; i.e., all these differential operators (or vector fields) on $P$ commute, and it is easy to check directly that $\left\{H_{a}, H_{b}\right\}=0$, i.e., that the corresponding Hamiltonian functions Poisson commute.

The transform, $G_{a}$, of the vector field $F_{a}(\partial)$ under the Fourier Transform is easy to compute: $G_{a}(w)(\alpha)=F_{a}(i \alpha) w(\alpha)$, or in words, the partial differential operator $F_{a}(\partial)$ is transformed by $\mathcal{F}$ into multiplication by the function $F_{a}(i \alpha)$. In "physicist language", this shows that the $G_{a}$ are all diagonal in the continuous basis for $P$ given by the evaluations $w \mapsto w(\alpha)$.

Before going on to consider the Scattering Transform we should mention another classical and elementary transform - one linearizing Burgers' Equation, $v_{t}=v_{x x}-$ $2 v v_{x}$. The transform, $\mathcal{C H}$ mapping $v$ to $w$, is $w=\exp \left(-\int v\right)$, and the inverse transform $\mathcal{I C H}$ that recovers $v$ from $w$ is $v=-\partial \log (w)=-\partial w / w$. Clearly $w$ must be positive for this to be defined, and it is easily checked that if $w$ is a positive solution of the linear heat conduction (or diffusion) equation $w_{t}=w_{x x}$, then $v$ satisfies Burgers' Equation. So if we start with any positive integrable function $w(x, 0)$, we can use the Fourier Transform method to find $w(x, t)$ satisfying the heat equation, and then $v(x, t)=-w_{x}(x, t) / w(x, t)$ will give a solution of Burgers' Equation. ( $\mathrm{CH}$ is usually referred to as the Cole-Hopf Transform, but the fact that it linearizes Burgers' Equation was actually pointed out by Forsyth in 1906, four decades before Cole and Hopf each independently rediscovered it.)

1. Lax Equations: $\mathbf{K d V}$ as an Isospectral Flow. In discussing the Inverse Scattering Transform it will be useful to have available an interesting reinterpretation of the KdV equation as formulated by Peter Lax. Namely, if $u(x, t)$ is a solution of the $\mathrm{KdV}$ equation, and we consider the one-parameter family $L(t)$ of self-adjoint operators on $L^{2}(\mathbf{R})$ that are given by the Schrödinger operators with potentials $u(t)(x)=u(x, t)$ (i.e., $L(t) \psi(x)=-\frac{d^{2}}{d x^{2}} \psi(x)+u(x, t) \psi(x)$ ), then these operators are isospectral, and in fact unitarily equivalent. That is, there is a smooth one-parameter family $U(t)$ of unitary operators on $L^{2}(\mathbf{R})$ such that $U(0)=I$ and $L(t)=U(t) L(0) U(t)^{-1}$.

By the way, in the following it will be convenient to take $\mathrm{KdV}$ in the form $u_{t}-6 u u_{x}+u_{x x x}=0$.

Suppose we have a smooth one-parameter family $U(t)$ of unitary transformations of a Hilbert space $H$ with $U(0)=I . U_{t}(t)$, the derivative of $U(t)$, is a tangent vector at $U(t)$ of the group $\mathcal{U}(H)$ of unitary transformations of $H$, so $B(t)=U_{t}(t) U(t)^{-1}=$ $U_{t}(t) U(t)^{*}$ is a tangent vector to $\mathcal{U}(H)$ at the identity, $I$. Differentiating $U U^{*}=I$ gives $U_{t} U^{*}+U U_{t}^{*}=0$, and since $U_{t}=B U$ and $U_{t}^{*}=U^{*} B^{*}, 0=B U U^{*}+U U^{*} B^{*}$, so $B^{*}=-B$; i.e., $B(t)$ is a family of skew-adjoint operators on $H$. Conversely, 
a smooth map $t \mapsto B(t)$ of $\mathbf{R}$ into the skew-adjoint operators defines a timedependent right invariant vector field $X_{U}(t)=B(t) U$ on $\mathcal{U}(H)$ and so (at least in finite dimensions) a smooth curve $U(t)$ of unitary operators starting from $I$ such that $U_{t}(t)=B(t) U(t)$.

Now suppose that $L(0)$ is a self-adjoint operator on $H$, and define a family of conjugate operators $L(t)$ by $L(t)=U(t) L(0) U(t)^{-1}$, so $L(0)=U(t)^{*} L(t) U(t)$. Differentiating the latter with respect to $t, 0=U_{t}^{*} L U+U^{*} L_{t} U+U^{*} L U_{t}=$ $U^{*}\left(-B L+L_{t}+L B\right) U$. Hence, writing $[B, L]=B L-L B$ as usual for the commutator of $B$ and $L$, we see that $L(t)$ satisfies the so-called Lax Equation, $L_{t}=[B, L]$.

Given a smooth family of skew-adjoint operators $B(t)$, the Lax Equation is a time-dependent linear ODE in the vector space $\mathcal{S}$ of self-adjoint operators on $H$, whose special form expresses the fact that the evolution is by unitary conjugation. Indeed, since the commutator of a skew-adjoint operator and a self-adjoint operator is again self-adjoint, $B(t)$ defines a time-dependent vector field, $Y$, on $\mathcal{S}$ by $Y(t)(L)=[B(t), L]$. Clearly a smooth curve $L(t)$ in $\mathcal{S}$ satisfies the Lax Equation if and only if it is a solution curve of $Y$. By uniqueness of solutions of linear ODE, the solution $L(t)$ of this ODE with initial condition $L(0)$ must be the one-parameter family $U(t) L(0) U(t)^{-1}$ constructed above.

Given any $\psi(0)$ in $H$, define $\psi(t)=U(t) \psi(0)$. Since $U(t) L(0)=L(t) U(t)$, it follows that if $\psi(0)$ is an eigenvector of $L(0)$ belonging to the eigenvalue $\lambda$, then $\psi(t)$ is an eigenvalue of $L(t)$ belonging to the same eigenvalue $\lambda$. Differentiating the relation defining $\psi(t)$ gives $\psi_{t}=B \psi(t)$, so we may consider $\psi(t)$ to be defined as the solution of this linear ODE with initial value $\psi(0)$. Since this is one of the main ways in which we will use Lax Equations, we will restate it as what we shall call the:

Isospectral Principle. Let $L(t)$ and $B(t)$ be smooth one-parameter families of self-adjoint and skew-adjoint operators respectively on a Hilbert space $H$, satisfying the Lax Equation $L_{t}=[B, L]$, and let $\psi(t)$ be a curve in $H$ that is a solution of the time-dependent linear $O D E \psi_{t}=B \psi$. If the initial value, $\psi(0)$, is an eigenvector of $L(0)$ belonging to an eigenvalue $\lambda$, then $\psi(t)$ is an eigenvector of $L(t)$ belonging to the same eigenvalue $\lambda$.

Remark. There is a more general (but less precise) version of the Isospectral Principle that follows by an almost identical argument. Let $V$ be any topological vector space and $B(t)$ a family of linear operators on $V$ such that the evolution equation $U_{t}=B U$ is well-defined. This means that for each $\psi(0)$ in $V$ there should exist a unique solution to the time-dependent linear $\operatorname{ODE} \psi_{t}(t)=B(t) \psi(t)$. The evolution operator $U(t)$ is of course then defined by $U(t) \psi(0)=\psi(t)$, so $U_{t}=B U$. Then clearly the conclusion of the Isospectral Principle still holds. That is to say, if a smooth family of linear operators $L(t)$ on $V$ satisfies the Lax Equation $L_{t}=[B, L]$, then $U(t) L(0)=L(t) U(t)$, so if $L(0) \psi(0)=\lambda \psi(0)$, then $L(t) \psi(t)=\lambda \psi(t)$.

We now apply the above with $H=L^{2}(\mathbf{R})$. We will see that if $u$ satisfies $\mathrm{KdV}$, then the family of Schrödinger operators $L(t)$ on $H$ defined above satisfies the Lax Equation $L_{t}=[B, L]$, where

$$
B(t) \psi(x)=-4 \psi_{x x x}(x)+3\left(u(x, t) \psi_{x}(x)+(u(x, t) \psi(x))_{x}\right),
$$

or more succinctly, $B=-4 \partial^{3}+3(u \partial+\partial u)$. Here and in the sequel it is convenient to use the same symbol both for an element $w$ of the Schwartz space, $\mathcal{S}(\mathbf{R})$, and for the bounded self-adjoint multiplication operator $v \mapsto w v$ on $H$. Since $H$ is infinite 
dimensional and our operators $B$ and $L$ are unbounded on $H$, some care is needed for a rigorous treatment. But this is relatively easy. Note that all the operators involved have the Schwartz space as a common dense domain, so we can use the preceding remark taking $V=\mathcal{S}(\mathbf{R})$ (we omit details).

Note that since $\partial$ is skew-adjoint, so is any odd power, and in particular $4 \partial^{3}$ is skew-adjoint. Also, the multiplication operator $u$ is self-adjoint, while the anticommutator of a self-adjoint and a skew-adjoint operator is skew-adjoint, so $u \partial+\partial u$ and hence $B$ is indeed skew-adjoint.

Since clearly $L_{t}=u_{t}$, while $u_{t}-6 u u_{x}+u_{x x x}=0$ by assumption, to prove that $L_{t}=[B, L]$ we must check that $[B, L]=6 u u_{x}-u_{x x x}$. Now $[B, L]=4\left[\partial^{3}, \partial^{2}\right]-$ $4\left[\partial^{3}, u\right]-3\left[u \partial, \partial^{2}\right]+3[u \partial, u]-3\left[\partial u, \partial^{2}\right]+3[\partial u, u]$, and it easy to compute the six commutator relations $\left[\partial^{3}, \partial^{2}\right]=0,\left[\partial^{3}, u\right]=u_{x x x}+3 u_{x x} \partial+3 u_{x} \partial^{2},\left[u \partial, \partial^{2}\right]=$ $-u_{x x} \partial-2 u_{x} \partial^{2},[u \partial, u]=u u_{x},\left[\partial u, \partial^{2}\right]=-3 u_{x x} \partial-2 u_{x} \partial^{2}-u_{x x x}$, and $[\partial u, u]=$ $-u u_{x}$, from which the desired expression for $[B, L]$ is immediate.

Let us now apply the Isospectral Principle to this example.

KdV Isospectrality Theorem. Suppose $u(x, t)$ is a solution of the KdV equation,

$$
u_{t}-6 u u_{x}+u_{x x x}=0,
$$

whose initial value $u(x, 0)$ is in the Schwartz space $\mathcal{S}(\mathbf{R})$, and that $\psi(x)$ is an eigenfunction of the Schrödinger Equation with potential $u(x, 0)$ and eigenvalue $\lambda$ :

$$
-\frac{d^{2}}{d x^{2}} \psi(x)+u(x, 0) \psi(x)=\lambda \psi(x) .
$$

Let $\psi(x, t)$ be the solution of the evolution equation $\psi_{t}=B \psi$, i.e.,

$$
\frac{\partial \psi}{\partial t}=-4 \frac{\partial^{3} \psi}{\partial x^{3}}+3\left(u(x, t) \frac{\partial \psi}{\partial x}(x, t)+\frac{\partial}{\partial x}(u(x, t) \psi(x, t))\right)
$$

with the initial value $\psi(x, 0)=\psi(x)$. Then $\psi(x, t)$ is an eigenfunction for the Schrödinger Equation with potential $u(x, t)$ and the same eigenvalue $\lambda$ :

$$
-\psi_{x x}(x, t)+u(x, t) \psi(x, t)=\lambda \psi(x, t),
$$

and moreover, if $\psi(x)$ is in $L^{2}$, then the $L^{2}$ norm of $\psi(\cdot, t)$ is independent of $t$. Finally, $\psi(x, t)$ also satisfies the first-order evolution equation

$$
\psi_{t}-(4 \lambda+2 u) \psi_{x}+u_{x} \psi=0 .
$$

Proof. Except for the final statement this is an immediate application of the Isospectrality Principle. Differentiating the eigenvalue equation for $\psi(x, t)$ with respect to $x$ gives $\psi_{x x x}=u_{x} \psi+(u-\lambda) \psi_{x}$, and substituting this into the assumed evolution equation for $\psi$ gives the asserted first-order equation for $\psi$.

By the way, it should be emphasized that the essential point is that when a potential evolves via $\mathrm{KdV}$, then the corresponding Schrödinger operators are isospectral, and this is already clearly stated in [GGKM]. Lax's contribution was to explain the mechanism behind this remarkable fact and to formulate it in a way that was easy to generalize. In fact, almost all generalizations of the phenomena first recognized in KdV have used the Lax Equation as a jumping-off place. 
2. The Scattering Data and Its Evolution. We now fix a "potential function" $u$ in the Schwartz space $\mathcal{S}(\mathbf{R})$ and look more closely at the space $E_{\lambda}(u)$ of $\lambda$ eigenfunctions of the Schrödinger operator with this potential. By definition, $E_{\lambda}(u)$ is just the kernel of the linear operator $L^{u}(\psi)=-\frac{d^{2} \psi}{d x^{2}}+u \psi-\lambda \psi$ acting on the space $C^{\infty}(\mathbf{R})$, and by the elementary theory of second-order linear ODE it is, for each choice of $\lambda$, a two-dimensional linear subspace of $C^{\infty}(\mathbf{R})$. Using the special form of $L^{u}$ we can describe $E_{\lambda}(u)$ more precisely. We will ignore the case $\lambda=0$, and consider the case of positive and negative $\lambda$ separately.

Suppose $\lambda=-\kappa^{2}, \kappa>0$. Note that any $\psi$ in $E_{\lambda}(u)$ will clearly be of the form $\psi(x)=a e^{\kappa x}+b e^{-\kappa x}$ in any interval on which $u$ vanishes identically. Thus if $u$ has compact support, say $u(x)=0$ for $|x|>M$, then we can find a basis $\psi_{\lambda,-\infty}^{+}, \psi_{\lambda,-\infty}^{-}$for $E_{\lambda}(u)$ such that for $x<-M, \psi_{\lambda,-\infty}^{ \pm}(x)=e^{ \pm \kappa x}$, or equivalently $\psi_{\lambda,-\infty}^{+}(x) e^{-\kappa x}=1$ and $\psi_{\lambda,-\infty}^{-}(x) e^{\kappa x}=1$ for $x<-M$. Similarly there is a second basis $\psi_{\lambda, \infty}^{+}, \psi_{\lambda, \infty}^{-}$for $E_{\lambda}(u)$ such that $\psi_{\lambda, \infty}^{+}(x) e^{-\kappa x}=1$ and $\psi_{\lambda, \infty}^{-}(x) e^{\kappa x}=1$ for $x>M$. When $u$ does not have compact support but is only rapidly decreasing, then it can be shown that there still exist two bases $\psi_{\lambda,-\infty}^{+}, \psi_{\lambda,-\infty}^{-}$and $\psi_{\lambda, \infty}^{+}, \psi_{\lambda, \infty}^{-}$ for $E_{\lambda}(u)$ such that $\lim _{x \rightarrow-\infty} \psi_{\lambda,-\infty}^{+}(x) e^{-\kappa x}=1$ and $\lim _{x \rightarrow-\infty} \psi_{\lambda,-\infty}^{-}(x) e^{\kappa x}=1$, while $\lim _{x \rightarrow \infty} \psi_{\lambda, \infty}^{+}(x) e^{-\kappa x}=1$ and $\lim _{x \rightarrow \infty} \psi_{\lambda, \infty}^{-}(x) e^{\kappa x}=1$. (A more descriptive way of writing these limits is $\psi_{\lambda,-\infty}^{+}(x) \sim e^{\kappa x}$ and $\psi_{\lambda,-\infty}^{-}(x) \sim e^{-\kappa x}$ as $x \rightarrow-\infty$, while $\psi_{\lambda, \infty}^{+}(x) \sim e^{\kappa x}$ and $\psi_{\lambda, \infty}^{-}(x) \sim e^{-\kappa x}$ as $x \rightarrow \infty$.) Let us define functions $f(\lambda)$ and $c(\lambda)$ by $\psi_{\lambda,-\infty}^{+}=f(\lambda) \psi_{\lambda, \infty}^{+}+c(\lambda) \psi_{\lambda, \infty}^{-}$. Using these bases it is easy to detect when $\lambda$ is a so-called "discrete eigenvalue" of $L^{u}$, i.e., when $E_{\lambda}(u)$ contains a non-zero element $\psi$ of $L^{2}(\mathbf{R})$. We can assume $\psi$ has $L^{2}$ norm one, and since $\psi_{\lambda,-\infty}^{-}$blows up at $-\infty$ while $\psi_{\lambda, \infty}^{+}$blows up at $\infty, \psi$ must be both a multiple of $\psi_{\lambda,-\infty}^{+}$and of $\psi_{\lambda, \infty}^{-}$, and since $\psi \neq 0$ it follows that $f(\lambda)=0$. Conversely, if $f(\lambda)=0$, then $\psi_{\lambda,-\infty}^{+}=c(\lambda) \psi_{\lambda, \infty}^{-}$decays exponentially both at $\infty$ and $-\infty$ and so we can normalize it to get an element of $E_{\lambda}(u)$ with $L^{2}$ norm one. Thus the discrete eigenvalues of $L^{u}$ are precisely the roots of the function $f$.

It follows from standard arguments of Sturm-Liouville theory that in fact $L^{u}$ has only finitely many discrete eigenvalues, $\lambda_{1}, \ldots, \lambda_{N}$, with corresponding $L^{2}$ normalized eigenfunctions $\psi_{1}, \ldots, \psi_{N}$, and these determine so-called "normalization constants" $c_{1}, \ldots, c_{N}$ by $\psi_{n}=c_{n} \psi_{\lambda_{n}, \infty}^{-}$; i.e., if we write $\lambda_{n}=-\kappa_{n}^{2}$, then $c_{n}$ is characterized by $\psi_{n}(x) \sim c_{n} e^{-\kappa_{n} x}$ as $x \rightarrow \infty$. We note that the $\psi_{n}$ and hence the normalization constants $c_{n}$ are only determined up to sign, but we will only use $c_{n}^{2}$ in the Inverse Scattering Transform.

For $\lambda=k^{2}, k>0$ there are similar considerations. In this case, if $u(x)$ vanishes for $|x|>M$, then any element of $E_{\lambda}(u)$ will be of the form $a e^{i k x}+b e^{-i k x}$ for $x<-M$ and also of the form $c e^{i k x}+d e^{-i k x}$ for $x>M$. If $u$ is only rapidly decaying, then we can still find bases $\psi_{\lambda,-\infty}^{+}, \psi_{\lambda,-\infty}^{-}$and $\psi_{\lambda, \infty}^{+}, \psi_{\lambda, \infty}^{-}$for $E_{\lambda}(u)$ such that $\psi_{\lambda,-\infty}^{+}(x) \sim e^{i k x}$ and $\psi_{\lambda,-\infty}^{-}(x) \sim e^{-i k x}$ as $x \rightarrow-\infty$, while $\psi_{\lambda, \infty}^{+}(x) \sim e^{i k x}$ and $\psi_{\lambda, \infty}^{-}(x) \sim e^{-i k x}$ as $x \rightarrow \infty$. Then $\psi_{\lambda,-\infty}^{-}=\alpha \psi_{\lambda, \infty}^{-}+\beta \psi_{\lambda, \infty}^{+}$, where $\alpha$ can be shown to be non-zero. Dividing by $\alpha$ we get a particular eigenfunction $\psi_{k}$, called the Jost solution, with the special asymptotic behavior $\psi_{k}(x) \sim a(k) e^{-i k x}$ as $x \rightarrow-\infty$ and $\psi_{k}(x) \sim e^{-i k x}+b(k) e^{i k x}$ as $x \rightarrow \infty$. 
The functions $a(k)$ and $b(k)$ are called the transmission coefficient and reflection coefficient respectively, and $b(k)$ together with the above normalizing constants $c_{1}, \ldots c_{n}$ make up the "Scattering Data", $\mathcal{S}(u)$ for $u$.

While it is perhaps intuitively clear that the bases $\psi_{\lambda, \pm \infty}^{ \pm}$must exist, to supply the asymptotic arguments required for a rigorous proof of the crucial theorem on the time evolution of the Scattering Data it is essential to give them precise definitions, and we do this next.

First consider the simpler problem of the first order ODE $L^{u} \psi=\frac{d \psi}{d x}-u \psi$. If we make the substitution $\psi=e^{\lambda x} \phi$, then the eigenvalue equation $L^{u}(\psi)=\lambda \psi$ becomes $\frac{d \phi}{d x}=u \phi$, so (assuming $u$ depends on a parameter $t$ ) we have $\phi(x, t)=$ $\exp \left(\int_{-\infty}^{x} u(\xi, t) d \xi\right)$. Note that $\lim _{x \rightarrow-\infty} \phi(x, t)=1$ while

$$
\lim _{x \rightarrow \infty} \phi(x, t)=\exp \left(\int_{0}^{\infty} u(\xi, t) d \xi\right)=c(t),
$$

so if $\psi(x, t)$ is an eigenfunction of $L^{u}, \psi(x, t) \sim c(t) e^{\lambda x}$ (i.e., $\lim _{x \rightarrow \infty} \psi(x, t) e^{-\lambda x}=$ $c(t))$ and since $u(x, t)$ is rapidly decaying, we can moreover differentiate under the integral sign to obtain $\psi_{t}(x, t) \sim c^{\prime}(t) e^{\lambda x}$. One cannot differentiate asymptotic relations in general of course, and since we will need a similar relation for eigenfunctions of Schrödinger operators we must make a short detour to justify it by an argument similar to the above.

If we now make the substitution $\psi=\phi e^{-\kappa x}$ in the eigenvalue equation $\psi_{x x}=$ $\kappa^{2} \psi+u \psi$, then we get after simplifications $\phi_{x} x-2 \kappa \phi_{x}=u \phi$, or $\partial(\partial-2 \kappa) \phi=$ $u \phi$. Recall the method of solving the inhomogeneous equation $\partial(\partial-2 \kappa) \phi=f$ by "variation of parameters". Since 1 and $e^{2 \kappa x}$ form a basis for the solutions of the homogeneous equation, we look for a solution of the form $\phi=\Theta_{1}+\Theta_{2} e^{2 \kappa x}$, and to make the system determined we add the relation $\Theta_{1}^{\prime}+\Theta_{2}^{\prime} e^{2 \kappa x}=0$. This leads to the equations $\Theta_{1}^{\prime}=-\frac{f}{2 \kappa}$ and $\Theta_{2}^{\prime}=\frac{f}{2 \kappa} e^{2 \kappa x}$, so $\phi=-\frac{1}{2 \kappa} \int_{0}^{x} f(\xi) d \xi+$ $\frac{e^{2 \kappa x}}{2 \kappa} \int_{0}^{x} f(\xi) e^{-2 \kappa x} d \xi$. If we now take $f=u \phi$ (and use $\phi e^{-\kappa x}=\psi$ ), then we get the relation $\phi(x, t)=\frac{1}{2 \kappa} \int_{x}^{0} u(\xi, t) \phi(\xi, t) d \xi-\frac{e^{2 \kappa x}}{2 \kappa} \int_{x}^{0} u(\xi, t) \psi(\xi, t) e^{-\kappa x} d \xi$. Assuming

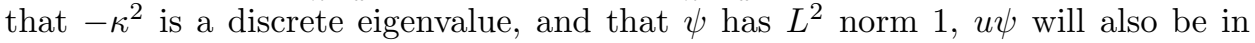
$L^{2}$, and we can estimate the second integral using the Schwartz Inequality, and we see that in fact $\left|\int_{x}^{0} u(\xi) \psi(\xi) e^{-\kappa x} d \xi\right|<O\left(e^{-\kappa x}\right)$, so the second term is $O\left(e^{\kappa x}\right)$. It follows that $\psi(x, t) \sim c(t) e^{\kappa x}$ in the sense that $\lim _{x \rightarrow-\infty} \psi(x, t) e^{-\kappa x}=c(t)$, where $c(t)=\phi(-\infty, t)=\frac{1}{2 \kappa} \int_{-\infty}^{0} u(\xi, t) \phi(\xi, t) d \xi$. In other words, the normalizing constant is well defined. But what is more important, it also follows that if $u(x, t)$ satisfies $\mathrm{KdV}$, then the normalizing constant $c(t)$ for a fixed eigenvalue $-\kappa^{2}$ is a differentiable function of $t$ and satisfies $\psi_{t}(x, t) \sim c^{\prime}(t) e^{\kappa x}$. This follows from the fact that we can differentiate the formula for $c(t)$ under the integral sign because $u$ is rapidly decreasing. Note that differentiating the relation $\psi e^{\kappa x}=\phi$ gives $\psi_{x} e^{\kappa x}=\phi_{x}-\kappa \psi$. But the formula for $\phi$ shows that $\phi_{x}$ converges to zero at $-\infty$, so $\psi_{x}(x, t) \sim-\kappa c(t) e^{\kappa x}$. From the KdV Isospectrality Theorem, we know that if $u(x, t)$ satisfies $\mathrm{KdV}$, then $\psi(x, t)$ satisfies $\psi_{t}-\left(-4 \kappa^{2}+2 u\right) \psi_{x}+u_{x} \psi=0$, so the left hand side times $e^{\kappa x}$ converges to $c^{\prime}(t)+4 \kappa^{2}(-\kappa c(t))$ as $x \rightarrow \infty$ and hence $c^{\prime}(t)-4 \kappa^{3} c(t)=0$, so $c(t)=c(0) e^{4 \kappa^{3} t}$.

By a parallel argument (which we omit) it follows that the transmission and reflection coefficients are also well defined and that the Jost solution $\psi_{k}(x, t)$ satisfies $\left(\psi_{k}\right)_{t} \sim a_{t}(k, t) e^{-i k x}$ at $-\infty$ and $\left(\psi_{k}\right)_{t} \sim b_{t}(k, t) e^{i k x}$ at $\infty$, and then one can 
show from the KdV Isospectrality Theorem that the transmission coefficients are constant, while the reflection coefficients satisfy $b(k, t)=b(k, 0) e^{8 i k^{3} t}$.

Theorem on Evolution of the Scattering Data. Let $u(t)=u(x, t)$ be $a$ smooth curve in $\mathcal{S}(\mathbf{R})$ satisfying the $K d V$ equation $u_{t}-6 u u_{x}+u_{x x x}=0$ and assume that the Schrödinger operator with potential u(t) has discrete eigenvalues $-\kappa_{1}^{2}, \ldots,-\kappa_{N}^{2}$ whose corresponding normalized eigenfunctions have normalization constants $c_{1}(t), \ldots, c_{n}(t)$. Let the transmission and reflection coefficients of $u(t)$ be respectively $a(k, t)$ and $b(k, t)$. Then the transmission coefficients are all constants of the motion, i.e., $a(k, t)=a(k, 0)$, while the Scattering Data, $c_{n}(t)$ and $b(k, t)$, satisfy:

1) $c_{n}(t)=c_{n}(0) e^{4 \kappa_{n}^{3} t}$

2) $b(k, t)=b(k, 0) e^{8 i k^{3} t}$.

We note a striking (and important) fact: not only do we now have an explicit and simple formula for the evolution of the scattering data $\mathcal{S}(u(t))$ when $u(t)$ evolves by the $\mathrm{KdV}$ equation, but further this formula does not require any knowledge of $u(t)$.

The fact that the transmission coefficients $a(k)$ are constants of the motion while the logarithms of the reflection coefficients $b(k)$ vary linearly with time suggests that perhaps they can somehow be regarded as action-angle variables for the $\mathrm{KdV}$ equation, thereby identifying $\mathrm{KdV}$ as a completely integrable system in a precise sense. While $a(k)$ and $b(k)$ are not themselves canonical variables, Zakharov and Fadeev in $[\mathrm{ZF}]$ showed that certain functions of $a$ and $b$ did satisfy the Poisson commutation relations for action-angle variables. Namely, the functions $p(k)=$ $(k / \pi) \log |a(k)|^{2}=(k / \pi) \log \left[1+|b(k)|^{2}\right]$ and $q(k)=\arg (b(k))$ satisfy $\left\{p(k), q\left(k^{\prime}\right)\right\}=$ $\delta\left(k-k^{\prime}\right)$ and $\left\{p(k), p\left(k^{\prime}\right)\right\}=\left\{q(k), q\left(k^{\prime}\right)\right\}=0$.

The above formula for the evolution of the Scattering Data is one of the key ingredients for The Inverse Scattering Method, and we are finally in a position to describe this elegant algorithm for solving the Cauchy problem for KdV.

\section{The Inverse Scattering Method}

To solve the $\mathrm{KdV}$ initial value problem $u_{t}-6 u u_{x}+u_{x x x}=0$ with given initial potential $u(x, 0)$ in $\mathcal{S}(\mathbf{R})$ :

1) Apply the "Direct Scattering Transform", i.e., find the discrete eigenvalues $-\kappa_{1}^{2}, \ldots,-\kappa_{N}^{2}$ for the Schrödinger operator with potential $u(x, 0)$ and compute the Scattering Data, i.e., the normalizing constants $c_{n}(0)$ and the reflection coefficients $b(k, 0)$.

2) Define $c_{n}(t)=c_{n}(0) e^{4 \kappa_{n}^{3} t}$ and $b(k, t)=b(k, 0) e^{8 i k^{3} t}$.

3) Use the Inverse Scattering Transform (described below) to compute $u(t)$ from $c_{n}(t)$ and $b(k, t)$.

3. The Inverse Scattering Transform. Recovering the potential $u$ of a Schrödinger operator $L^{u}$ from the Scattering Data $\mathcal{S}(u)$ was not something invented for the purpose of solving the KdV initial value problem. Rather, it was a question of basic importance to physicists doing Cyclotron experiments, and the theory was worked out in the mid-1950's by Kay and Moses [KM], Gelfand and Levitan [GL], and Marchenko [M]. 
Denote the discrete eigenvalues of $u$ by $-\kappa_{1}^{2}, \ldots,-\kappa_{N}^{2}$, the normalizing constants by $c_{1}, \ldots, c_{N}$, and the reflection coefficients by $b(k)$, and define a function

$$
B(\xi)=\sum_{n=1}^{N} c_{n}^{2} e^{-\kappa_{n} \xi}+\frac{1}{2 \pi} \int_{-\infty}^{\infty} b(k) e^{i k \xi} d k .
$$

Inverse Scattering Theorem. The potential $u$ can be recovered using the formula $u(x)=-2 \frac{d}{d x} K(x, x)$, where $K(x, z)$ is the unique function on $\mathbf{R} \times \mathbf{R}$ that is zero for $z<x$ and satisfies the Gelfand-Levitan-Marchenko Integral Equation:

$$
K(x, z)+B(x+z)+\int_{-\infty}^{\infty} K(x, y) B(y+z) d y=0 .
$$

(For a proof, see [DJ], Chapter 3, Section 3, or [La3], Chapter II.)

We will demonstrate by example how the Inverse Scattering Method can now be applied to get explicit solutions of KdV. But first a couple of general remarks about solving the Gelfand-Levitan-Marchenko equation. We assume in the following that $B$ is rapidly decreasing.

Let $C(\mathbf{R} \times \mathbf{R})$ denote the Banach space of bounded, continuous real-valued functions on $\mathbf{R} \times \mathbf{R}$ with the sup norm. Define $\mathcal{F}^{B}: C(\mathbf{R} \times \mathbf{R}) \rightarrow C(\mathbf{R} \times \mathbf{R})$ by the formula

$$
\mathcal{F}^{B}(K)(x, z)=-B(x+z)-\int_{-\infty}^{\infty} K(x, y) B(y+z) d y .
$$

Then $K$ satisfies the Gelfand-Levitan-Marchenko equation if and only if it is a fixedpoint of $\mathcal{F}^{B}$. It is clear that $\mathcal{F}^{B}$ is Lipschitz with constant $\|B\|_{L^{1}}$, so if $\|B\|_{L^{1}}<1$, then by the Banach Contraction Principle the Gelfand-Levitan-Marchenko equation has a unique solution, and it is the limit of the sequence $K_{n}$ defined by $K_{1}(x, z)=$ $-B(x+z), K_{n+1}=\mathcal{F}^{B}\left(K_{n}\right)$.

Secondly, we note that if the function $B$ is "separable" in the sense that it satisfies an identity of the form $B(x+z)=\sum_{n=1}^{N} X_{n}(x) Z_{n}(z)$, then the GelfandLevitan-Marchenko equation takes the form

$$
K(x, z)+\sum_{n=1}^{N} X_{n}(x) Z_{n}(z)+\sum_{n=1}^{N} Z_{n}(z) \int_{x}^{\infty} K(x, y) X_{n}(y) d y=0 .
$$

It follows that $K(x, z)$ must have the form $K(x, z)=\sum_{n=1}^{N} L_{n}(x) Z_{n}(z)$. If we substitute this for $K$ in the previous equation and define $a_{n m}(x)=\int_{x}^{\infty} Z_{m}(y) X_{n}(y) d y$, then we have reduced the problem to solving $N$ linear equations for the unknown functions $L_{n}$, namely: $L_{n}(x)+X_{n}(x)+\sum_{m=1}^{N} a_{n m}(x) L_{m}(x)=0$, or $X_{n}(x)+$ $\sum_{m=1}^{N} A_{n m}(x) L_{m}(x)=0$, where $A_{n m}(x)=\delta_{n m}+a_{n m}(x)$. Thus finally we have

$$
K(x, x)=-\sum_{n=1}^{N} Z_{n}(x) \sum_{m=1}^{N} A_{n m}^{-1}(x) X_{m}(x) .
$$

4. An Explicit Formula for KdV Multi-Solitons. A potential $u$ is called "reflectionless" if all the reflection coefficients are zero. Because of the relation $b(k, t)=b(k, 0) e^{8 i k^{3} t}$, it follows that if $u(x, t)$ evolves by $\mathrm{KdV}$ and if it is reflectionless at $t=0$, then it is reflectionless for all $t$. If the discrete eigenvalues of such a potential are $-\kappa_{1}^{2}, \ldots,-\kappa_{N}^{2}$ and the normalizing constants are $c_{1}, \ldots, c_{N}$, then $B(\xi)=\sum_{n=1}^{N} c_{n}^{2} e^{-\kappa_{n} \xi}$, so $B(x+z)=\sum_{n=1}^{N} X_{n}(x) Z_{n}(z)$, where $X_{n}(x)=c_{n}^{2} e^{-\kappa_{n} x}$, and $Z_{n}(z)=e^{-\kappa_{n} z}$ and we are in the separable case just considered. Recall that 
$a_{n m}(x)=\int_{x}^{\infty} Z_{m}(y) X_{n}(y) d y=c_{n}^{2} \int_{x}^{\infty} e^{-\left(\kappa_{n}+\kappa_{m}\right) y} d y=c_{n}^{2} e^{-\left(\kappa_{n}+\kappa_{m}\right) x} /\left(\kappa_{n}+\kappa_{m}\right)$, and that

$$
A_{n m}(x)=\delta_{n m}+a_{n m}(x)=\delta_{n m}+c_{n}^{2} e^{-\left(\kappa_{n}+\kappa_{m}\right) x} /\left(\kappa_{n}+\kappa_{m}\right) .
$$

Differentiation gives $\frac{d}{d x} A_{n m}(x)=-c_{n}^{2} e^{-\left(\kappa_{n}+\kappa_{m}\right) x}$, so by a formula above

$$
\begin{aligned}
K(x, x) & =-\sum_{n=1}^{N} Z_{n}(x) \sum_{m=1}^{N} A_{n m}^{-1}(x) X_{m}(x) \\
& =\sum_{n=1}^{N} e^{-\kappa_{n} x} \sum_{m=1}^{N} A_{n m}^{-1}(x)\left(-c_{m}^{2} e^{-\kappa_{m} x}\right) \\
& =\sum_{n=1}^{N} \sum_{m=1}^{N} A_{n m}^{-1} \frac{d}{d x} A_{m n}(x) \\
& =\operatorname{tr}\left(A^{-1}(x) \frac{d}{d x} A(x)\right) \\
& =\frac{1}{\operatorname{det}(A(x))} \frac{d}{d x} \operatorname{det} A(x) \\
& =\frac{d}{d x} \log \operatorname{det} A(x),
\end{aligned}
$$

and so $u(x)=-2 \frac{d}{d x} K(x, x)=-2 \frac{d^{2}}{d x^{2}} \log \operatorname{det} A(x)$.

If $N=1$ and we put $\kappa=\kappa_{1}$, it is easy to see that this formula reduces to our earlier formula for traveling-wave solutions of the $\mathrm{KdV}$ equation: $u(x, t)=$ $-\frac{\kappa^{2}}{2} \operatorname{sech}^{2}\left(\kappa\left(x-\kappa^{2} t\right)\right)$. We can also use it to find explicit solutions $u(x, t)$ for $N=2$. Let $g_{i}(x, t)=\exp \left(\kappa_{i}^{3} t-\kappa_{i} x\right)$, and set $A=\frac{\left(\kappa_{1}-\kappa_{2}\right)^{2}}{\left(\kappa_{1}+\kappa_{2}\right)^{2}}$. Then

$$
u(x, t)=-2 \frac{\kappa_{1}^{2} g_{1}+\kappa_{2}^{2} g_{2}+2\left(\kappa_{1}-\kappa_{2}\right)^{2} g_{1} g_{2}+A g_{1} g_{2}\left(\kappa_{1}^{2} g_{2}+\kappa_{2}^{2} g_{1}\right)}{\left(1+g_{1}+g_{2}+A g_{1} g_{2}\right)^{2}} .
$$

For general $N$ the solutions $u(x, t)$ that we get this way are referred to as the pure $N$-soliton solutions of the $\mathrm{KdV}$ equation. It is not hard to show by an asymptotic analysis that for large negative and positive times they behave as a superposition of the above traveling-wave solutions, and that after the larger, faster moving waves have all passed through the slower moving shorter ones and they have become wellseparated, the only trace of their interactions are certain predictable "phase-shifts", i.e., certain constant translations of the locations of their maxima from where they would have been had they not interacted. (For details see [L], p.123.)

5. The KdV Hierarchy. By oversimplifying a bit, one can give a succinct statement of what makes the KdV equation, $u_{t}-6 u u_{x}+u_{x x x}$, more than just a run-ofthe-mill evolution equation; namely it is equivalent to a Lax equation, $L_{t}^{u}=\left[B, L^{u}\right]$, expressing that the corresponding Schrödinger operator $L^{u}=-\frac{d^{2}}{d x^{2}}+u$ is evolving by unitary equivalence - so that the spectral data for $L^{u}$ provides many constants of the motion for $\mathrm{KdV}$, and in fact enough commuting constants of the motion to make KdV completely integrable.

It is natural to ask whether $\mathrm{KdV}$ is unique in that respect, and the answer is a resounding "No!". In his paper introducing the Lax Equation formulation of KdV, [La1], Peter Lax already pointed out an important generalization. Recall that $B=-4 \partial^{3}+3(u \partial+\partial u)$. Lax suggested that for each integer $j$ one should 
look for an operator of the form $B_{j}=\alpha \partial^{2 j+1}+\sum_{i=1}^{j}\left(b_{i} \partial^{2 i-1}+\partial^{2 i-1} b_{i}\right)$, where the operators $b_{i}$ are to be chosen so as to make the commutator $\left[B_{j}, L^{u}\right]$ a zero order operator - that is $\left[B_{j}, L^{u}\right]$ should be multiplication by some polynomial, $K_{j}(u)$, in $u$ and its derivatives. This requirement imposes $j$ conditions on the $j$ coefficients $b_{i}$, and these conditions uniquely determine the $b_{i}$ as multiplications by certain polynomials in $u$ and its derivatives. For example, $B_{0}=\partial$, and the corresponding Lax Equation $u_{t}=K_{0}(u)$ is $u_{t}=u_{x}$, the so-called Linear Advection Equation. And of course $B_{1}$ is just our friend $-4 \partial^{3}+3(u \partial+\partial u)$, whose corresponding Lax Equation is $\mathrm{KdV}$.

$K_{j}(u)$ is a polynomial in the derivatives of $u$ up through order $2 j+1$, and the evolution equation $u_{t}=K_{j}(u)$ is referred to as the $j$-th higher order KdV equation. This whole sequence of flows is known as "The KdV Hierarchy", and the initial value problem for each of these equations can be solved using the Inverse Scattering Method in a straightforward generalization from the KdV case. But even more remarkably:

Theorem. Each of the higher order KdV equations defines a Hamiltonian flow on $P$. That is, for each positive integer $j$ there is a Hamiltonian function $F_{j}: P \rightarrow \mathbf{R}$ (defined by a polynomial differential operator of order $j, \tilde{F}\left(u_{(0)}, \ldots, u_{(j)}\right)$ ) such that $K_{j}(u)=\left(\nabla_{s} F_{j}\right)_{u}$. Moreover, all the functions $F_{j}$ are in involution, so that all the higher order KdV flows commute with each other.

The proof can be found in [La3], Chapter I.

It should be pointed out here that the discovery of the constants of the motion $F_{k}$ goes back to the earliest work on $\mathrm{KdV}$ as an integrable system. In fact, it came out of the research in 1966 by Gardner, Greene, Kruskal, and Miura leading up to their paper $[\mathrm{GGKM}]$ in which the Inverse Scattering Method was introduced. However, the symplectic structure for the phase space of $\mathrm{KdV}$, and the fact that these functions were in involution, was only discovered considerably later, in 1971 $[\mathrm{G}],[\mathrm{ZF}]$.

To the best of my knowledge, the higher order KdV equations are not of independent interest. Nevertheless, the above theorem suggests a subtle but important change in viewpoint towards the $\mathrm{KdV}$ equation - one that proved important in further generalizing the Inverse Scattering Method to cover other evolution equations which are of interest for their own sake. Namely, the key player in the Inverse Scattering Method should not be seen as the KdV equation itself, but rather the Schrödinger operator $L^{u}$. If we want to generalize the Inverse Scattering Method, we should first find other operators $L$ with a "good scattering theory" and then look among the Lax Equations $L_{t}=[M, L]$ to find interesting candidates for integrable systems that can be solved using scattering methods.

In fact, this approach has proved important in investigating both finite and infinite dimensional Hamiltonian systems, and in the remainder of this article we will investigate in detail one such scheme that has not only been arguably the most successful in identifying and solving important evolution equations, but has moreover a particularly elegant and powerful mathematical framework that underlies it. This scheme was first introduced by Zakharov and Shabat [ZS] to study an important special equation (the so-called Nonlinear Schrödinger Equation, or NLS). Soon thereafter, Ablowitz, Kaup, Newell, and Segur [AKNS1] showed that one relatively minor modification of the Zakharov and Shabat approach recovers the theory of the $\mathrm{KdV}$ equation, while another leads to an Inverse Scattering Theory analysis for a 
third very important evolution equation, the Sine-Gordon Equation (SGE). AKNS went on to develop the Zakharov and Shabat technique into a general method for $\mathrm{PDE}$ with values in $2 \times 2$-matrix groups [AKNS2], and ZS further generalized it to the case of $n \times n$-matrix groups. Following current custom, we will refer to this method as the ZS-AKNS Scheme.

\section{The ZS-AKNS SChEME}

1. Flat Connections and the Lax Equation, ZCC. To prepare for the introduction of the ZS-AKNS Scheme, we must first develop some of the infra-structure on which it is based. This leads quickly to the central Lax Equation of the theory, the so-called "Zero-Curvature Condition" (or ZCC).

First we fix a matrix Lie Group $\mathbf{G}$ and denote its Lie algebra by $\mathcal{G}$. That is, $\mathbf{G}$ is some closed subgroup of the group $\mathbf{G L}(n, \mathbf{C})$ of all $n \times n$ complex matrices, and $\mathcal{G}$ is the set of all $n \times n$ complex matrices, $X$, such that $\exp (X)$ is in $\mathbf{G}$. If you feel more comfortable working with a concrete example, think of $\mathbf{G}$ as the group $\mathbf{S L}(n, \mathbf{C})$ of all $n \times n$ complex matrices of determinant 1 , and $\mathcal{G}$ as its Lie algebra $\operatorname{sl}(n, \mathbf{C})$ of all $n \times n$ complex matrices of trace zero. In fact, for the original ZSAKNS Scheme, $\mathbf{G}=\mathbf{S L}(2, \mathbf{C})$ and $\mathcal{G}=\mathbf{s l}(2, \mathbf{C})$, and we will carry out most of the later discussion with these choices, but for what we will do next the precise nature of $\mathbf{G}$ is irrelevant.

Let $\boldsymbol{\nabla}$ be a flat connection for the trivial principal bundle $\mathbf{R}^{2} \times \mathbf{G}$. Then we can write $\boldsymbol{\nabla}=d-\omega$, where $\omega$ is a 1 -form on $\mathbf{R}^{2}$ with values in the Lie algebra $\mathcal{G}$. Using coordinates $(x, t)$ for $\mathbf{R}^{2}$ we can then write $\omega=A d x+B d t$ where $A$ and $B$ are smooth maps of $\mathbf{R}^{2}$ into $\mathcal{G}$.

If $X$ is a vector field on $\mathbf{R}^{2}$, then the covariant derivative operator in the direction $X$ is $\nabla_{X}=\partial_{X}-\omega(X)$, and in particular, the covariant derivatives in the coordinate directions $\frac{\partial}{\partial x}$ and $\frac{\partial}{\partial t}$ are $\nabla_{\frac{\partial}{\partial x}}=\frac{\partial}{\partial x}-A$ and $\nabla_{\frac{\partial}{\partial t}}=\frac{\partial}{\partial t}-B$.

Since we are assuming that $\nabla$ is flat, it determines a global parallelism. If $\left(x_{0}, t_{0}\right)$ is any point of $\mathbf{R}^{2}$, then we have a map $\psi: \mathbf{R}^{2} \rightarrow \mathbf{G}$, where $\psi(x, t)$ is the parallel translation operator from $\left(x_{0}, t_{0}\right)$ to $(x, t)$. Considered as a section of our trivial principal bundle, $\psi$ is covariant constant, i.e., $\nabla_{X} \psi=0$ for any tangent vector field $X$. In particular, taking $X$ to be $\frac{\partial}{\partial x}$ and $\frac{\partial}{\partial t}$ gives the relations $\psi_{x}=A \psi$ and $\psi_{t}=B \psi$.

There are many equivalent ways to express the flatness of the connection $\nabla$. On the one hand the curvature 2-form $d \omega-\omega \wedge \omega$ is zero. Equivalently, the covariant derivative operators in the $\frac{\partial}{\partial x}$ and $\frac{\partial}{\partial t}$ directions commute, i.e., $\left[\frac{\partial}{\partial x}-A, \frac{\partial}{\partial t}-B\right]=$ 0 , or finally, equating the cross-derivatives of $\psi,(A \psi)_{t}=\psi_{x t}=\psi_{t x}=(B \psi)_{x}$. Expanding the latter gives $A_{t} \psi+A \psi_{t}=B_{x} \psi+B \psi_{x}$ or $A_{t} \psi+A B \psi=B_{x} \psi+B A \psi$, and right multiplying by $\psi^{-1}$ we arrive at the so-called "Zero-Curvature Condition": $A_{t}-B_{x}-[A, B]=0$. Rewriting this as $-A_{t}=-B_{x}+[B,-A]$, and noting that $\left[B, \frac{\partial}{\partial x}\right]=-B_{x}$, we see that the Zero-Curvature Condition has an equivalent formulation as a Lax Equation:

$$
\left(\frac{\partial}{\partial x}-A\right)_{t}=\left[B, \frac{\partial}{\partial x}-A\right]
$$

and it is ZCC that plays the central rôle in the ZS-AKNS Scheme.

Recall what ZCC is telling us. If we look at $t$ as a parameter, then the operator $\frac{\partial}{\partial x}-A\left(x, t_{0}\right)$ is the covariant derivative in the $x$-direction along the line $t=t_{0}$, and 
the Lax Equation ZCC says that as a function of $t_{0}$ these operators are all conjugate. Moreover the operator $\psi\left(t_{0}, t_{1}\right)$ implementing the conjugation between the time $t_{0}$ and the time $t_{1}$ satisfies $\psi_{t}=B \psi$, which means it is parallel translation from $\left(x, t_{0}\right)$ to $\left(x, t_{1}\right)$ computed by going "vertically" along the curve $t \mapsto(x, t)$. But since $\frac{\partial}{\partial x}-A\left(x, t_{0}\right)$ generates parallel translation along the horizontal curve $x \mapsto\left(x, t_{0}\right)$, what this amounts to is the statement that parallel translating horizontally from $\left(x_{0}, t_{0}\right)$ to $\left(x_{1}, t_{0}\right)$ is the same as parallel translation vertically from $\left(x_{0}, t_{0}\right)$ to $\left(x_{0}, t_{1}\right)$ followed by parallel translation horizontally from $\left(x_{0}, t_{1}\right)$ to $\left(x_{1}, t_{1}\right)$ followed by parallel translation vertically from $\left(x_{1}, t_{1}\right)$ to $\left(x_{1}, t_{0}\right)$. Thus, in the case of ZCC, the standard interpretation of the meaning of a Lax Equation reduces to a special case of the theorem that if a connection has zero curvature, then the holonomy around a contractible path is trivial.

2. Some ZS-AKNS Examples. The ZS-AKNS Scheme is a method for solving the initial value problem for certain (hierarchies of) evolution equations on a space of "potentials" $P$. In general $P$ will be of the form $\mathcal{S}(\mathbf{R}, V)$, where $V$ is some finite dimensional real or complex vector space, i.e., each potential $u$ will be a map $x \mapsto u(x)$ of Schwartz class from $\mathbf{R}$ into $V$. (A function $u$ with values in $V$ is of Schwartz class if, for each linear functional $\ell$ on $V$, the scalar valued function $\ell \circ u$ is of Schwartz class, or equivalently if, when we write $u$ in terms of a fixed basis for $V$, its components are of Schwartz class.) The evolution equations in question are of the form $u_{t}=F(u)$ where the map $F: P \rightarrow P$ is a "polynomial differential operator" -i.e., it has the form $F(u)=p\left(u, u_{x}, u_{x x}, \ldots\right)$, where $p$ is a polynomial mapping of $V$ to itself.

When we say we want to solve the initial value (or "Cauchy") problem for such an equation, we of course mean that given $u^{0}=u(x, 0)$ in $P$ we want to find a smooth map $t \mapsto u(t)=u(x, t)$ of $\mathbf{R}$ to $P$ with $u(0)=u^{0}$ and $u_{t}(x, t)=$ $p\left(u(x, t), u_{x}(x, t), u_{x x}(x, t), \ldots\right)$. In essence, we want to think of $F$ as a vector field on $P$ and construct the flow $\phi_{t}$ that it generates. (Of course, if $P$ were a finite dimensional manifold, then we could construct the flow $\phi_{t}$ by solving a system of ODE's, and as we shall see, the ZS-AKNS Scheme allows us in certain cases to solve the PDE $u_{t}=p\left(u, u_{x}, u_{x x}, \ldots\right)$ by reducing it to ODE's.)

The first and crucial step in using the ZS-AKNS Scheme to study a particular such evolution equation consists in setting up an interpretation of $A$ and $B$ so that the equation $u_{t}=p\left(u, u_{x}, u_{x x}, \ldots\right)$ becomes a special case of ZCC.

To accomplish this, we first identify $V$ with a subspace of $\mathcal{G}$ (so that $P=\mathcal{S}(\mathbf{R}, V)$ becomes a subspace of $\mathcal{S}(\mathbf{R}, \mathcal{G}))$, and define a map $u \mapsto A(u)$ of $P$ into $C^{\infty}(\mathbf{R}, \mathcal{G})$ of the form $A(u)=$ const $+u$, so that if $u$ depends parametrically on $t$, then $\left(\frac{\partial}{\partial x}-A(u)\right)_{t}=-u_{t}$.

Finally (and this is the difficult part) we must define a map $u \mapsto B(u)$ of $P$ into $C^{\infty}(\mathbf{R}, \mathcal{G})$ so that $\left[B(u), \frac{\partial}{\partial x}-A(u)\right]=-p\left(u, u_{x}, u_{x x}, \ldots\right)$.

To interpret the latter equation correctly, and in particular to make sense out of the commutator bracket in a manner consistent with our earlier interpretation of $A$ and $B$, it is important to be clear about the interpretation $A(u)$ and $B(u)$ as operators, and in particular to be precise about the space on which they are operating. This is just the space $C^{\infty}(\mathbf{R}, \mathbf{g l}(2, \mathbf{C}))$ of smooth maps $\psi$ of $\mathbf{R}$ into the space of all complex $2 \times 2$ matrices. Namely, we identify $A(u)$ with the zeroorder differential operator mapping $\psi$ to $A(u) \psi$, the pointwise matrix product of $A(u)(x)$ and $\psi(x)$, and similarly with $B(u)$. (This is a complete analogy with the 
KdV situation, where in interpreting the Schrödinger operator, we identified our potential $u$ with the operator of multiplication by $u$.) Of course $\left(\frac{\partial}{\partial x} \psi\right)(x)=\psi_{x}$.

We will now illustrate this with three examples: the KdV equation, the Nonlinear Schrödinger Equation (NLS), and the Sine-Gordon Equation (SGE). In each case $V$ will be a one-dimensional space that is embedded in the space of off-diagonal complex matrices $\left(\begin{array}{ll}0 & b \\ c & 0\end{array}\right)$, and in each case $A(u)=\mathbf{a} \lambda+u$, where $\lambda$ is a complex parameter, and $\mathbf{a}$ is the constant, diagonal, trace zero matrix $\mathbf{a}=\left(\begin{array}{cc}-i & 0 \\ 0 & i\end{array}\right)$.

Example 1.. [AKNS1] Take $u(x)=\left(\begin{array}{cc}0 & q(x) \\ -1 & 0\end{array}\right)$, and let

$$
B(u)=\mathbf{a} \lambda^{3}+u \lambda^{2}+\left(\begin{array}{cc}
\frac{i}{2} q & \frac{i}{2} q_{x} \\
0 & -\frac{i}{2} q
\end{array}\right) \lambda+\left(\begin{array}{cc}
\frac{q_{x}}{4} & \frac{-q^{2}}{2} \\
\frac{q}{2} & -\frac{q_{x}}{4}
\end{array}\right) .
$$

Then an easy computation shows that ZCC is satisfied if and only if $q$ satisfies $\mathrm{KdV}$ in the form $q_{t}=-\frac{1}{4}\left(6 q q_{x}+q_{x x x}\right)$.

Example 2. [ZS] Take $u(x)=\left(\begin{array}{cc}0 & q(x) \\ -\bar{q}(x) & 0\end{array}\right)$, and let

$$
B(u)=\mathbf{a} \lambda^{2}+u \lambda+\left(\begin{array}{cc}
\frac{i}{2}|q|^{2} & \frac{i}{2} q_{x} \\
-\frac{i}{2} \bar{q}_{x} & -\frac{i}{2}|q|^{2}
\end{array}\right) .
$$

In this case ZCC is satisfied if and only if $q(x, t)$ satisfies the so-called Nonlinear Schrödinger Equation (NLS) $q_{t}=\frac{i}{2}\left(q_{x x}+2|q|^{2} q\right)$.

Example 3. [AKNS1] Take $u=\left(\begin{array}{cc}0 & -\frac{q_{x}(x)}{2} \\ \frac{q_{x}(x)}{2} & 0\end{array}\right)$, and let $B(u)=\frac{1}{\lambda} v$ where $v(x)=\frac{i}{4}\left(\begin{array}{cc}\cos q(x) & \sin q(x) \\ \sin q(x) & -\cos q(x)\end{array}\right)$. In this case, ZCC is satisfied if and only if $q$ satisfies the Sine-Gordon Equation (SGE) in the form $q_{x t}=\sin q$.

In the following description of the ZS-AKNS Scheme, we will state definitions and describe constructions in a way that works for the general ZS-AKNS case - and we will even make occasional remarks explaining what modifications are necessary to extend the theory to the more general case of $n \times n$ matrix groups. (For the full details of this latter generalization the reader should consult [Sa].) However, working out details in even full ZS-AKNS generality would involve many distracting detours to discuss various special situations that are irrelevant to the main ideas. So, for ease and clarity of exposition, we will carry out most of the further discussion of the ZS-AKNS Scheme within the framework of the NLS Hierarchy.

3. The Uses of Solitons. There are by now dozens of "soliton equations", but not only were the three examples from the preceding section the first to be discovered, they are also the best known, and in many ways still the most interesting and important. In fact, in addition to their simplicity and their Hamiltonian nature, each has certain special properties that give them a "universal" character, so that they are almost sure to arise as approximate models in any physical situation that exhibits these properties. In this section I will try to say a little about these special features, and also explain how these equations have been used in both theoretical and applied mathematics. 
We have already discussed in some detail the historical background and many of the interesting features and applications of the $\mathrm{KdV}$ equation, so here I will only re-iterate the basic property responsible for its frequent appearance in applied problems. In the KdV equation there is an extraordinary balance between the shock-forming tendency of its non-linear term $u u_{x}$ and the dispersive tendency of its linear term $u_{x x x}$, and this balance is responsible for the existence of remarkably stable configurations (solitons) that scatter elastically off one another under the $\mathrm{KdV}$ evolution. Moreover KdV is the simplest non-dissipative wave-equation with these properties.

The Sine-Gordon equation is even older than the KdV equation; it arose first in the mid-nineteenth century as the master equation for "pseudo-spherical surfaces" (i.e., surfaces of constant negative Gaussian curvature immersed in $\mathbf{R}^{3}$ ). Without going into the details (cf. [Da] and [PT], Part I, Section 3.2), the Gauss-Codazzi equations for such surfaces reduce to the Sine-Gordon equation, so that by the "Fundamental Theorem of Surface Theory", there is a bijective correspondence between isometry classes of isometric immersions of the hyperbolic plane into $\mathbf{R}^{3}$ and solutions to the Sine-Gordon equation. (Strictly speaking, we relax the immersion condition to admit cusp singularities along curves.) Because of this (and the great interest in non-Euclidean geometry during the latter half of the last century) a prodigious amount of effort was devoted to the study of the Sine-Gordon equation by the great geometers of that period, resulting in a beautiful body of results, most of which can be found in G. Darboux' superb treatise on surface theory Leçons sur la Théorie Générale des Surfaces [Da].

One of the most notable features of this theory is the concept of a "Bäcklund transformation". Starting from any solution of the Sine-Gordon equation, this creates a two-parameter family of new solutions. One slight complication is that the construction of the new solutions requires solving a certain ordinary differential equation. However the so-called "Bianchi Permutability Formula" allows us to easily compose Bäcklund transformations. That is, once we have found this first set of new solutions, we can apply another Bäcklund transformation to any one of them to get still more solutions of Sine-Gordon, and this second family of new solutions can be written down explicitly as algebraic functions of the first set, without solving any more ODEs. Moreover, we can continue inductively in this manner, getting an infinite sequence of families of more and more complex solutions to the Sine-Gordon equations (and related pseudospherical surfaces). If we take as our starting solution the identically zero (or "vacuum") solution to the SineGordon equation, this process can be carried out explicitly. At the first stage we get the so-called Kink (or one-soliton) solutions to the Sine-Gordon equation, and the corresponding family of pseudospherical surfaces is the Dini family (including the well-known pseudosphere). Using the Bianchi Formula once gives rise to the twosoliton solutions of Sine-Gordon and the corresponding Küen Surface, and repeated application leads in principle to all the higher soliton solutions of the Sine-Gordon equations (cf. [Da], [PT], loc. cit. for more details). In fact, the classical geometers knew so much about the "soliton sector" of solutions to Sine-Gordon that it might seem surprising at first that they did not go on to discover "soliton mathematics" a century before it actually was. But of course they knew only half the storythey knew nothing of the dispersive, non-soliton solutions to Sine-Gordon and had no imaginable way to discover the Inverse Scattering Transform, which is the key to a full understanding of the space of all solutions. (And finally, they probably 
never looked at Sine-Gordon as an evolution equation for a one-dimensional wave, so they did not notice the strange scattering behavior of the solutions that they had calculated.)

Nevertheless, their work did not go in vain. As soon as it was realized that Sine-Gordon was a soliton equation, it was natural to ask whether KdV also had an analogous theory of Bäcklund transformations that, starting from the vacuum solution, marched up the soliton ladder. It was quickly discovered that this was in fact so, and while Bäcklund transformations have remained until recently one of the more mysterious parts of soliton theory, each newly discovered soliton equation was found to have an associated theory of Bäcklund transformations. Indeed this soon came to be considered a hallmark of the "soliton syndrome", and a test that one could apply to detect soliton behavior. A natural explanation of this relationship follows from the Terng-Uhlenbeck Loop Group approach to soliton theory, and we will remark on it briefly at the end of this article. For full details see [TU2].

The Sine-Gordon equation has also been proposed as a simplified model for a unified field theory, and derived as the equation governing the propogation of dislocations in a crystal lattice, the propogation of magnetic flux in a Josephson junction transmission line, and many other physical problems.

The Nonlinear Schrödinger Equation has an interesting pre-history. It was discovered "in disguise" (and then re-discovered at least three times, cf. [Ri]) in the early part of this century. In 1906, Da Rios wrote a master's thesis [DaR] under the direction of Levi-Civita, in which he modeled the free evolution of a thin vortex-filament in a viscous liquid by a time-dependent curve $\gamma(x, t)$ in $\mathbf{R}^{3}$ satisfying the equation $\gamma_{t}=\gamma_{x} \times \gamma_{x x}$. Now by the Frenet equations, $\gamma_{x} \times \gamma_{x x}=\kappa B$ where $\kappa=\kappa(x, t)$ is the curvature and $B$ the binormal, so the filament evolves by moving in the direction of its binormal with a speed equal to its curvature. This is now often called the "vortex-filament equation" or the "smoke-ring equation". In 1971, Hasimoto noticed a remarkable gauge transformation that transforms the vortex-filament equation to the Nonlinear Schrödinger equation. In fact, if $\tau(\cdot, t)$ denotes the torsion of the curve $\gamma(\cdot, t)$, then the complex quantity $q(x, t)=\kappa(x, t) \exp \left(i \int \tau(\xi, t) d \xi\right)$ satisfies NLS if and only if $\gamma$ satisfies the vortexfilament equation.

But it is as an "envelope equation" that NLS has recently come into its own. If a one-dimensional, amplitude modulated, high-frequency wave is moving in a highly dispersive and non-linear medium, then to a good approximation the evolution of the wave envelope (i.e., the modulating signal) in a coordinate system moving at the group velocity of the wave will satisfy NLS. Without going into detail about what these hypotheses mean (cf. [HaK]) they do in fact apply to the light pulses travelling along optical fibers that are rapidly becoming the preferred means of communicating information at high bit-rates over long distances. Soliton solutions of NLS seem destined to play a very important rôle in keeping the Internet and the World Wide Web from being ruined by success. The story is only half-told at present, but the conclusion is becoming clear and it is too good a story to omit.

For over a hundred years, analogue signals travelling over copper wires provided the main medium for point-to-point communication between humans. Early implementations of this medium (twisted pair) were limited in bandwidth (bits per second) to about $100 \mathrm{~Kb} / \mathrm{s}$ per channel. By going over to digital signalling instead of analogue, one can get up to the $1 \mathrm{Mb} / \mathrm{s}$ range, and using coaxial cable one can 
squeeze out another several orders of magnitude. Until recently this seemed sufficient. A bandwidth of about $1 \mathrm{~Gb} / \mathrm{s}$ is enough to satisfy the needs of the POTS (plain old telephone system) network that handles voice communication for the entire United States, and that could be handled with coaxial cable and primitive fiber optic technology for the trunk lines between central exchanges, and twisted pairs for the low bandwidth "last mile" from the exchange to a user's home. And as we all know, a coaxial cable has enough bandwidth to provide us with several hundred channels of television coming into our homes.

But suddenly all this has changed. As more and more users are demanding very high data-rate services from the global Internet, the capacities of the communication providers have been stretched to and beyond their limits, and they have been desperately trying to keep up. The problem is particularly critical in the transoceanic links joining North America to Asia and Europe. Fortunately, a lot of fiber optic cables have been laid down in the past decade, and even more fortunately these cables are being operated at bandwidths that are very far below their theoretical limits of about $100 \mathrm{~GB} / \mathrm{s}$. To understand the problems involved in using these resources more efficiently, it is necessary to understand how a bit is transmitted along an optical fiber. In principle it is very simple. In so-called RZ (return-to-zero) coding, a pulse of high-frequency laser-light is sent to indicate a one, or not sent to indicate a zero. The inverse of the pulse-width in seconds determines the maximum bandwidth of the channel. A practical lower bound for the pulse-width is about a pico-second $\left(10^{-12}\right.$ seconds), giving an upper bound of about $1000 \mathrm{~GB} / \mathrm{s}$ for the bandwidth. But of course there are further practical difficulties that limit data-rates to well below that figure (e.g., the pulses should be well-separated, and redundancy must be added for error correction), but actual data transmission rates over optical fibers in the $100 \mathrm{~GB} / \mathrm{s}$ range seem to be a reasonable goal (using wavelength-division-multiplexing).

But there are serious technical problems. Over-simplifying somewhat, a major obstacle to attaining such rates is the tendency of these very short pico-second pulses to disperse as they travel down the optical fiber. For example, if an approximate square-wave pulse is sent, then dispersion will cause very high error rates after only several hundreds of miles. However if the pulses are carefully shaped to that of an appropriate NLS soliton, then the built-in stability of the soliton against dispersion will preserve the pulse shape over very long distances, and theoretical studies show that error-free propogation at $10 \mathrm{~GB} / \mathrm{s}$ across the Pacific is feasible with current technology, even without multi-plexing. (For further details and references see $[\mathrm{LA}]$.)

4. Nonlinear Schrödinger as a Hamiltonian Flow. Let $\mathbf{G}$ denote the group $\mathbf{S U}(2)$ of unitary $2 \times 2$ complex matrices of determinant 1 , and $\mathcal{G}$ its Lie algebra, $\mathbf{s u}(2)$, of skew-adjoint complex matrices of trace 0 . The 3 -dimensional real vector space $\mathcal{G}$ has a natural positive definite inner product (the Killing form), defined by $<<a, b\rangle>=-\frac{1}{2} \operatorname{tr}(a b)$. It is characterized (up to a constant factor) by the fact that it is "Ad-invariant", i.e., if $g \in \mathbf{G}$, then $<<\operatorname{Ad}(g) a, \operatorname{Ad}(g) b\rangle\rangle=\langle\langle a, b\rangle\rangle$, where $\operatorname{Ad}(g): \mathcal{G} \rightarrow \mathcal{G}$ is defined by $\operatorname{Ad}(g) a=g a g^{-1}$. Equivalently, for each element $c$ of $\mathcal{G}$, $\operatorname{ad}(c): \mathcal{G} \rightarrow \mathcal{G}$ defined by $\operatorname{ad}(c) a=[c, a]$ is skew-adjoint with respect to the Killing form: $\langle<[c, a], b>>+<<a,[c, b]\rangle>=0$.

We denote by $\mathbf{T}$ the standard maximal torus of $\mathbf{G}$, i.e., the group $\operatorname{diag}\left(e^{-i \theta}, e^{i \theta}\right)$ of diagonal, unitary matrices of determinant 1 , and $\mathcal{T}$ will denote its Lie algebra 
$\operatorname{diag}(-i \theta, i \theta)$ of skew-adjoint, diagonal matrices of trace zero. We define the specific element $\mathbf{a}$ of $\mathcal{T}$ by $\mathbf{a}=\operatorname{diag}(-i, i)$.

The orthogonal complement, $\mathcal{T}^{\perp}$, of $\mathcal{T}$ in $\mathcal{G}$ will play an important rôle in what follows. It is clear that $\mathcal{T}^{\perp}$ is just the space of "off-diagonal" skew-adjoint matrices, i.e., those with all zeros on the diagonal. (This follows easily from the fact that the product of a diagonal matrix and an "off-diagonal" matrix is again off-diagonal, and so of trace zero.) Thus $\mathcal{T}^{\perp}$ is the space of matrices of the form $\left(\begin{array}{cc}0 & q \\ -\bar{q} & 0\end{array}\right)$ where $q \in \mathbf{C}$, and this gives a natural complex structure to the 2 -dimensional real vector space $\mathcal{T}^{\perp}$.

Note that $\mathcal{T}$ is just the kernel (or zero eigenspace) of ad(a). Since ad(a) is skewadjoint with respect to the Killing form, it follows that ad(a) leaves $\mathcal{T}^{\perp}$ invariant, and we will denote ad(a) restricted to $\mathcal{T}^{\perp}$ by $J: \mathcal{T}^{\perp} \rightarrow \mathcal{T}^{\perp}$. A trivial calculation shows that $J\left(\begin{array}{cc}0 & q \\ -\bar{q} & 0\end{array}\right)=\left(\begin{array}{cc}0 & 2 i q \\ -\overline{2 i q} & 0\end{array}\right)$.

Remark. In the generalization to $\mathbf{S U}(n)$, we choose a to be a diagonal element of $\operatorname{su}(n)$ that is "regular", i.e., has distinct eigenvalues. Then the Lie algebra $\mathcal{T}$ of the maximal torus $\mathbf{T}$ (the diagonal subgroup of $\mathbf{S U}(n)$ ) is still all diagonal skewadjoint operators of trace zero and is again the null-space of ad(a). Its orthogonal complement, $\mathcal{T}^{\perp}$, in $\mathbf{s u}(n)$ is thus still invariant under $\operatorname{ad}(\mathbf{a})$, but now it is no longer a single complex eigenspace, but rather the direct sum of complex ad (a) eigenspaces (the so-called "root spaces").

We define the phase space $P$ for the NLS Hierarchy by $P=\mathcal{S}\left(\mathbf{R}, \mathcal{T}^{\perp}\right)$, i.e., $P$ consists of all "potentials" $u$ that are Schwartz class maps of $\mathbf{R}$ into $\mathcal{T}^{\perp}: x \mapsto$ $u(x)=\left(\begin{array}{cc}0 & q(x) \\ -\bar{q}(x) & 0\end{array}\right)$. Clearly $u \mapsto q$ establishes a canonical identification of $P$ with the space $\mathcal{S}(\mathbf{R}, \mathbf{C})$ of all complex-valued Schwartz class functions on the line. We define an $L^{2}$ inner product on $P$, making it into a real pre-hilbert space, by $\left\langle u_{1}, u_{2}\right\rangle=\int_{-\infty}^{\infty}<<u_{1}(x), u_{2}(x)>>d x=-\frac{1}{2} \int_{-\infty}^{\infty} \operatorname{tr}\left(u_{1}(x) u_{2}(x)\right) d x$. When this is written in terms of $q$, we find $\left\langle u_{1}, u_{2}\right\rangle=\operatorname{Re}\left(\int_{-\infty}^{\infty} q_{1}(x) \overline{q_{2}(x)} d x\right)$. And finally, if we decompose $q_{1}$ and $q_{2}$ into their real and imaginary parts: $q_{j}=v_{j}+i w_{j}$, then $\left\langle u_{1}, u_{2}\right\rangle=\int_{-\infty}^{\infty}\left(v_{1} v_{2}+w_{1} w_{2}\right) d x$.

We "extend" $J: \mathcal{T}^{\perp} \rightarrow \mathcal{T}^{\perp}$ to act pointwise on $P$, i.e., $(J u)(x)=J(u(x))$, and since $J$ is skew-adjoint, we can define a skew bilinear form $\Omega$ on $P$ by

$$
\begin{aligned}
\Omega\left(u_{1}, u_{2}\right) & =\left\langle J^{-1} u_{1}, u_{2}\right\rangle \\
& =\operatorname{Re}\left(\int_{-\infty}^{\infty} \frac{1}{2 i} q_{1} \overline{q_{2}} d x\right) \\
& =-\frac{1}{2} \operatorname{Re}\left(i \int_{-\infty}^{\infty} q_{1} \overline{q_{2}} d x\right) \\
& =\frac{1}{2} \operatorname{Im}\left(\int_{-\infty}^{\infty} q_{1} \overline{q_{2}} d x\right) .
\end{aligned}
$$

Considered as a differential 2-form on the real topological vector space $P, \Omega$ is constant and hence closed. On the other hand, since $J: P \rightarrow P$ is injective, it follows that $\Omega$ is weakly non-degenerate, and hence a symplectic structure on $P$. 
From the definition of $\Omega$ we have $\Omega\left(J u_{1}, u_{2}\right)=\left\langle u_{1}, u_{2}\right\rangle$; thus if $F: P \rightarrow P$ has a Riemannian gradient $\boldsymbol{\nabla} F$, then $\Omega\left(J(\nabla F)_{u_{1}}, u_{2}\right)=\left\langle(\nabla F)_{u_{1}}, u_{2}\right\rangle=d F_{u_{1}}\left(u_{2}\right)$, and so $\nabla_{s} F=J \nabla F$. In particular, if $F_{1}$ and $F_{2}$ are any two Hamiltonian functions on $P$, then their Poisson bracket is given by the formula $\left\{F_{1}, F_{2}\right\}=\Omega\left(\nabla_{s} F_{2}, \nabla_{s} F_{1}\right)=$ $\Omega\left(J \nabla F_{2}, \nabla F_{1}\right)=\left\langle\nabla F_{2}, J \nabla F_{1}\right\rangle=\left\langle J \nabla F_{1}, \nabla F_{2}\right\rangle$.

A Calculus of Variations functional on $P, F: P \rightarrow \mathbf{R}$, will be of the form $F(u)=\int_{-\infty}^{\infty} \tilde{F}\left(v, w, v_{x}, w_{x}, \ldots\right) d x$, where $q=v+i w$, and the differential of $F$ is given by $d F_{u}(\delta u)=\int_{-\infty}^{\infty}\left(\frac{\delta F}{\delta v} \delta v+\frac{\delta F}{\delta w} \delta w\right) d x$, or equivalently

$$
d F_{u}(\delta u)=\frac{1}{2} \operatorname{Re}\left(\int_{-\infty}^{\infty}\left(\frac{\delta F}{\delta v}+i \frac{\delta F}{\delta w}\right)(\delta v-i \delta w) d x\right)
$$

where as usual $\frac{\delta F}{\delta v}=\frac{\partial \tilde{F}}{\partial v}-\frac{\partial}{\partial x}\left(\frac{\partial \tilde{F}}{\partial v_{x}}\right)+\frac{\partial^{2}}{\partial x^{2}}\left(\frac{\partial \tilde{F}}{\partial v_{x x}}\right)-\ldots$, and a similar expression for $\frac{\delta F}{\delta w}$. However, it will be more convenient to give the polynomial differential operator $\tilde{F}$ as a function of $q=u+i v, \bar{q}=u-i v, q_{x}=u_{x}+i v_{x}, \bar{q}_{x}=u_{x}-i v_{x}, \ldots$ instead of as a function of $u, v$ and their derivatives. Since $v=\frac{1}{2}(q+\bar{q})$ and $w=\frac{1}{2 i}(q-\bar{q})$, by the chain-rule, $\frac{\partial \tilde{F}}{\partial \bar{q}}=\frac{1}{2}\left(\frac{\partial \tilde{F}}{\partial v}+i \frac{\partial \tilde{F}}{\partial w}\right)$, with similar formulas for $\frac{\partial \tilde{F}}{\partial \bar{q}_{x}}, \frac{\partial \tilde{F}}{\partial \bar{q}_{x x}}$, etc. Thus if we define $\frac{\delta F}{\delta \bar{q}}=\frac{\partial \tilde{F}}{\partial \bar{q}}-\frac{\partial}{\partial x}\left(\frac{\partial \tilde{F}}{\partial \bar{q}_{x}}\right)+\frac{\partial^{2}}{\partial x^{2}}\left(\frac{\partial \tilde{F}}{\partial \bar{q}_{x x}}\right)-\ldots$, then $\frac{\delta F}{\delta \bar{q}}=\frac{\delta F}{\delta v}+i \frac{\delta F}{\delta w}$, and it follows that $d F_{u}(\delta u)=\frac{1}{2} \operatorname{Re}\left(\int_{-\infty}^{\infty} \frac{\delta F}{\delta \bar{q}} \overline{\delta q} d x\right)$, where $\delta q=\delta v+i \delta w$, so $\delta u=\left(\begin{array}{cc}0 & \delta q \\ -\overline{\delta q} & 0\end{array}\right)$. Recalling the formulae for $\left\langle u_{1}, u_{2}\right\rangle$, it follows that $\nabla F_{u}=\left(\begin{array}{cc}0 & \frac{\delta F}{\delta \bar{q}} \\ -\frac{\delta F}{\delta \bar{q}} & 0\end{array}\right)$, and so $\nabla_{s} F_{u}=\left(\begin{array}{cc}\frac{0}{-2 i \frac{\delta F}{\delta \bar{q}}} & 2 i \frac{\delta F}{\delta \bar{q}} \\ 0\end{array}\right)$. Thus, expressed in terms of $q$, the Hamiltonian flow in $P$ defined by $F$ is $q_{t}=2 i \frac{\delta F}{\delta \bar{q}}$.

If we take $F(u)=-\frac{1}{2} \operatorname{tr}\left(u^{4}+u_{x}^{2}\right)=\frac{1}{2}\left(|q|^{4}+\left|q_{x}\right|^{2}\right)$, then $\tilde{F}\left(q, \bar{q}, q_{x}, \bar{q}_{x}\right)=\frac{1}{2}\left(q^{2} \bar{q}^{2}+\right.$ $\left.q_{x} \bar{q}_{x}\right)$ and $\frac{\delta F}{\delta \bar{q}}=q^{2} \bar{q}+\frac{1}{2} \frac{\partial}{\partial x}\left(q_{x}\right)=\left(\frac{1}{2} q_{x x}+\left|q^{2}\right| q\right)$, and the Hamiltonian equation is $q_{t}=i\left(q_{x x}+2\left|q^{2}\right| q\right)$, which is NLS.

5. The Nonlinear Schrödinger Hierarchy. For each potential $u$ in $P$ and complex number $\lambda$ we define an element $A(u, \lambda)$ of $C^{\infty}(\mathbf{R}, \mathbf{s l}(2, \mathbf{C}))$ by $A(u, \lambda)=$ $\mathbf{a} \lambda+u=\left(\begin{array}{cc}-i \lambda & q \\ -\bar{q} & i \lambda\end{array}\right) . A(u, \lambda)$ will play an important rôle in what follows, and you should think of it as a zero-order differential operator on $C^{\infty}(\mathbf{R}, \mathbf{g l}(n, \mathbf{C}))$, acting by pointwise multiplication on the left. We are now going to imitate the construction of the KdV Hierarchy. That is, we will look for a sequence of maps $u \mapsto B_{j}(u, \lambda)$ of $P$ into $C^{\infty}(\mathbf{R}, \mathbf{s l}(2, \mathbf{C})$ ) (polynomials of degree $j$ in $\lambda$ ) such that the sequence of ZCC Lax Equations $u_{t}=\left[B_{j}, \frac{\partial}{\partial x}-A\right]$ is a sequence of commuting Hamiltonian flows on $P$, which for $j=2$ is the NLS flow.

NLS Hierarchy Theorem. For each $u$ in $P$ there exists a sequence of smooth maps $Q_{k}(u): \mathbf{R} \rightarrow \mathbf{s u}(2)$ with the following properties:

a) The $Q_{k}(u)$ can be determined recursively by:

i) $Q_{0}(u)$ is the constant matrix $\mathbf{a}$.

ii) $\left[\mathbf{a}, Q_{k+1}(u)\right]=\left(Q_{k}(u)\right)_{x}+\left[Q_{k}(u), u\right]$,

iii) $\left(Q_{k}(u)\right)_{x}+\left[Q_{k}(u), u\right]$ is off-diagonal. 
b) If we define $B_{j}(u, \lambda)=\sum_{k=0}^{j} Q_{k}(u) \lambda^{k-j}$, and consider $B_{k}(u, \lambda)$ as a zeroorder linear differential operator acting by pointwise matrix multiplication on elements $\psi$ of $C^{\infty}(\mathbf{R}, \mathbf{g l}(2, \mathbf{C}))$, then the conditions ii) and iii) of a) are equivalent to demanding that the commutators $\left[B_{j}(u, \lambda), \frac{\partial}{\partial x}-A(u, \lambda)\right]$ are independent of $\lambda$ and have only off-diagonal entries. In fact these commutators have the values:

$$
\left[B_{j}(u, \lambda), \frac{\partial}{\partial x}-A(u, \lambda)\right]=\left[\mathbf{a}, Q_{j+1}(u)\right]=\left(Q_{j}(u)\right)_{x}-\left[u, Q_{j}(u)\right] .
$$

c) The matrix elements of $Q_{k}(u)$ can be determined so that they are polynomials in the derivatives (up to order $k-1$ ) of the matrix entries of $u$, and this added requirement makes them uniquely determined. We can then regard $Q_{k}$ as a map of $P$ into $C^{\infty}(\mathbf{R}, \mathbf{s u}(2))$. Similarly, for each real $\lambda, u \mapsto B_{j}(u, \lambda)$ is a map of $P$ into $C^{\infty}(\mathbf{R}, \mathbf{s u}(2))$.

d) If follows that the sequence of ZCC Lax Equations, $\left(\frac{\partial}{\partial x}-A\right)_{t}=\left[B_{j}, \frac{\partial}{\partial x}-A\right]$, (or equivalently $u_{t}=\left[\mathbf{a}, Q_{j+1}(u)\right]$ ) determines flows on $P$, the so-called higher order NLS flows. (The $j$-th of these is called the $j$-th NLS flow and the second is the usual NLS flow).

e) If we define Hamiltonians on $P$ by $H_{k}(u)=-\frac{1}{k+1} \int_{-\infty}^{\infty} \operatorname{tr}\left(Q_{k+2}(u) \mathbf{a}\right) d x$, then $\left(\nabla H_{k}\right)_{u}$ is the off-diagonal part of $Q_{k+1}(u)$.

f) It follows that the $j$-th NLS flow is Hamiltonian, and in fact is given by $u_{t}=\left(\nabla_{s} H_{k}\right)_{u}$.

g) The Hamiltonian functions $H_{k}$ are in involution; i.e., the Poisson brackets $\left\{H_{k}, H_{l}\right\}$ all vanish, so that all the NLS flows on $P$ commute.

Remark. We will give part of the proof of this important theorem here, and finish the proof later when we have developed more machinery. However first we comment on the changes that are necessary when we go from 2 to $n$ dimensions (i.e., replace $\mathbf{g l}(2, \mathbf{C})$ by $\mathbf{g l}(n, \mathbf{C})$, and $\mathbf{s u}(2)$ by $\mathbf{s u}(n))$. In fact, surprisingly few changes are necessary. The maximal torus $\mathbf{T}$ still consists of diagonal unitary matrices of trace 1 but now has dimension $(n-1)$ rather than 1 . We replace a by any regular element of $\mathbf{T}$ (i.e., one with distinct elements on the diagonal). This is equivalent to the key condition that $\mathbf{T}$ is the commutator of $\mathbf{a}$. The biggest change is that to get the family of commuting Hamiltonian flows we must now choose a second element $\mathbf{b}$ of $\mathbf{T}$, and replace $Q_{j}(u)=Q_{\mathbf{a}, j}(u)$ by the more general $Q_{\mathbf{b}, j}(u)$, and the $B_{j}(u, \lambda)=B_{\mathbf{a}, j}(u, \lambda)$ by the more general $B_{\mathbf{b}, j}(u, \lambda)=\sum_{j=0}^{j} Q_{\mathbf{b}, k}(u) \lambda^{k-j}$. The only further change is that i) of a) now reads " $Q_{b, 0}(u)$ is the constant matrix b." Mutatis mutandis, everything else remains the same. For full details, see [Sa].

Proof. Some easier parts of the proof will be indicated here, while other more difficult steps will be deferred until after we discuss the ZS-AKNS direct scattering theory, at which point they will be much easier to demonstrate.

The coefficient of $\lambda^{j-k}$ in the commutator $\left[B_{j}(u, \lambda), \frac{\partial}{\partial x}-A(u, \lambda)\right]$ is easily computed, and for $k=0$ to $j-1$ we find $-\left(Q_{k}(u)\right)_{k}-\left[Q_{k}(u), u\right]-\left[Q_{k+1}(u), \mathbf{a}\right]$, while for $k=j$ (i.e., the term independent of $\lambda$ ) we get $-\left(Q_{j}(u)\right)_{x}-\left[Q_{j}(u), u\right]$, and c) is now immediate.

If we write $Q_{k}(u)$ as the sum of its diagonal part, $T_{k}(u)$, and its off-diagonal part, $P_{k}(u)$, then since ad(a) annihilates diagonal matrices and is an isomorphism on the off-diagonal matrices,

$$
\left[\mathbf{a}, Q_{k+1}(u)\right]=\operatorname{ad}(\mathbf{a})\left(T_{k+1}(u)\right)+\operatorname{ad}(\mathbf{a})\left(P_{k+1}(u)\right)=\operatorname{ad}(\mathbf{a})\left(P_{k+1}(u)\right),
$$


so by ii) of a):

$$
P_{k+1}(u)=\operatorname{ad}(\mathbf{a})^{-1}\left(\left(P_{k}(u)\right)_{x}+\left[T_{k}(u), u\right]\right) .
$$

(We have used the fact that, since $u$ is off-diagonal, $\left[u, T_{k}(u)\right]$ is off-diagonal while $\left[u, P_{k}(u)\right]$ is diagonal.)

Next note that condition iii) of statement a) can now be written as $\left(T_{j}(u)\right)_{x}=$ $\left[u, P_{j}(u)\right]$ (because $\left[u, P_{j}(u)\right]$ is diagonal while $\left[u, T_{j}(u)\right]$ is off-diagonal). So we can write

$$
T_{k+1}(u)=\int_{-\infty}^{x}\left[u, P_{k+1}(u)\right] d x
$$

where of course the indefinite integral is to be taken matrix element by matrix element. Together, the latter two displayed equations give an explicit recursive definition of $Q_{k+1}=P_{k+1}+T_{k+1}$ in terms of $Q_{k}=P_{k}+T_{k}$.

For example, since $Q_{0}(u)=\mathbf{a}$ we conclude that $P_{0}(u)=0$ and $T_{0}(u)=\mathbf{a}$. Then the formula for $P_{k+1}$ gives $P_{1}(u)=\operatorname{ad}(\mathbf{a})^{-1}(0+[\mathbf{a}, u])=u$, and since $[u, u]=0$, the formula for $T_{k+1}$ gives $T_{1}(u)=0$, and therefore $Q_{1}(u)=P_{1}(u)=u$.

Continuing, we find next that $P_{2}(u)=\operatorname{ad}(\mathbf{a})^{-1}\left(u_{x}\right)=\left(\begin{array}{cc}0 & -\frac{i}{2} q_{x} \\ \frac{i}{2} \bar{q}_{x} & 0\end{array}\right)$, and $\left(T_{2}(u)\right)_{x}=\left[u, P_{2}(u)\right]=\left(\begin{array}{cc}\frac{i}{2}\left(q_{x} \bar{q}+q \bar{q}_{x}\right) & 0 \\ 0 & -\frac{i}{2}\left(q_{x} \bar{q}+q \bar{q}_{x}\right)\end{array}\right)$, which gives by integration $T_{2}(u)=\left(\begin{array}{cc}\frac{i}{2}|q|^{2} & 0 \\ 0 & -\frac{i}{2}|q|^{2}\end{array}\right)$ and $Q_{2}(u)=P_{2}(u)+T_{2}(u)=\left(\begin{array}{cc}\frac{i}{2}|q|^{2} & -\frac{i}{2} q_{x} \\ \frac{i}{2} \bar{q}_{x} & -\frac{i}{2}|q|^{2}\end{array}\right)$. (By what we have seen earlier, this shows that the second flow is indeed the NLS flow.)

We could continue for another several steps, and at each stage, after computing $P_{j}(u)$ and then $\left[P_{j}(u), u\right]$, the anti-derivative of the latter turns out to be in $\mathcal{S}(\mathbf{R}, \mathcal{T})$, so $Q_{j}(u)=P_{j}(u)+T_{j}(u)$ is in $\mathcal{S}(\mathbf{R}, \mathbf{s u}(2))$. (Note that this is clearly equivalent to the statement that $\int_{-\infty}^{\infty}\left[u, P_{k+1}(u)\right] d x=0$.)

Unfortunately, no one has come up with a simple inductive proof of that fact, so at this stage we are faced with the unpleasant possibility that our recursive process might lead to some $T_{j}(u)$ (and hence $Q_{j}(u)$ ) that does not vanish at infinity. Later on, after we have discussed the scattering theory for the ZS-AKNS Scheme, we will find a simple argument to show that this cannot happen, and at that point we will have a proof of statements a) through d). Similarly, I do not know a proof of statement e) that avoids scattering theory, so I will again defer the proof.

Recalling that $\operatorname{ad}(\mathbf{a})$ (i.e., bracketing with a) annihilates diagonal matrices, it follows from e) that $\nabla_{s} H_{k}=J\left(\nabla H_{k}\right)=\left[\mathbf{a}, Q_{k+1}\right]$, and so by d) the $j$-th NLS flow is given by $u_{t}=\left(\nabla_{s} H_{k}\right)_{u}$, which is $\left.\mathrm{f}\right)$.

For g), recall $\left\{H_{k}, H_{l}\right\}=\left\langle J \nabla H_{k}, \nabla H_{l}\right\rangle=\left\langle\left[\mathbf{a}, Q_{k+1}(u)\right], Q_{l+1}(u)\right\rangle$, and using this formula, the ad-invariance of the Killing form, and the recursion relation $\left[\mathbf{a}, Q_{j+1}(u)\right]=\left(Q_{j}(u)\right)_{x}-\left[u, Q_{j}(u)\right]$, we will give an inductive argument that the $H_{k}$ are in involution.

Lemma 1. a) $\left\langle\left[u, Q_{j}(u)\right], Q_{k}(u)\right\rangle+\left\langle Q_{j}(u),\left[u, Q_{k}(u)\right]\right\rangle=0$.

b) $\left\langle\left[u, Q_{j}(u)\right], Q_{j}(u)\right\rangle=0$.

c) $\left\langle\left(Q_{j}(u)\right)_{x}, Q_{k}(u)\right\rangle+\left\langle Q_{j}(u),\left(Q_{k}(u)\right)_{x}\right\rangle=0$.

d) $\left\langle\left(Q_{j}(u)\right)_{x}, Q_{j}(u)\right\rangle=0$.

e) $\left\{H_{j}, H_{j-1}\right\}=0$. 
Proof. Statement a) is just a special case of the ad invariance of the Killing form, and $b$ ) is a special case of a).

Recalling that $\left\langle u_{1}, u_{2}\right\rangle=-\int_{-\infty}^{\infty} \operatorname{tr}\left(u_{1}, u_{2}\right) d x$, it follows that

$$
\left\langle\left(Q_{j}(u)\right)_{x}, Q_{k}(u)\right\rangle+\left\langle Q_{j}(u),\left(Q_{k}(u)\right)_{x}\right\rangle=-\int_{-\infty}^{\infty} \frac{d}{d x} \operatorname{tr}\left(Q_{j}(u), Q_{k}(u)\right) d x,
$$

which is clearly zero since $\operatorname{tr}\left(Q_{j}(u), Q_{k}(u)\right)$ vanishes at infinity. This proves c), and d) is just a special case of c).

Since $\left\{H_{j}, H_{j-1}\right\}=\left\langle\left[\mathbf{a}, Q_{j+1}(u)\right], Q_{j}(u)\right\rangle$, the recursion formula for $\left[\mathbf{a}, Q_{j+1}(u)\right]$ gives $\left\{H_{j}, H_{j-1}\right\}=\left\langle\left(Q_{j}(u)\right)_{x}, Q_{j}(u)\right\rangle-\left\langle\left[u, Q_{j}(u)\right], Q_{j}(u)\right\rangle$, and e) now follows from b) and d).

Lemma 2. $\left\{H_{k}, H_{l}\right\}=-\left\{H_{k-1}, H_{l+1}\right\}$.

Proof. $\left\{H_{k}, H_{l}\right\}=\left\langle\left[\mathbf{a}, Q_{k+1}(u)\right], Q_{l+1}(u)\right\rangle$, so that using the recursion formula for $\left[\mathbf{a}, Q_{k+1}(u)\right]$ we find:

$$
\left\{H_{k}, H_{l}\right\}=\left\langle\left(Q_{k}(u)\right)_{x}, Q_{l+1}(u)\right\rangle-\left\langle\left[u, Q_{k}(u)\right], Q_{l+1}(u)\right\rangle,
$$

and using a) of Lemma 1,

$$
\left\{H_{k}, H_{l}\right\}=\left\langle\left(Q_{k}(u)\right)_{x}, Q_{l+1}(u)\right\rangle+\left\langle\left(Q_{k}(u),\left[u, Q_{l+1}(u)\right]\right\rangle .\right.
$$

Next, using the recursion formula for $\left[a, Q_{l+2}(u)\right]$, we find that $\left\{H_{k}, H_{l}\right\}=$ $\left\langle\left(Q_{k}(u)\right)_{x}, Q_{l+1}(u)\right\rangle+\left\langle\left(Q_{k}(u),\left(Q_{l+1}(u)\right)_{x}\right\rangle-\left\langle\left(Q_{k}(u),\left[a, Q_{l+2}(u)\right]\right\rangle\right.\right.$, and we recognize the third term as $-\left\{H_{k+1}, H_{l+1}\right\}$, while the sum of the first two terms vanishes by c) of Lemma 1 .

The proof that $\left\{H_{k}, H_{l}\right\}=0$ for any $k$ and $l$ is now easy. We can suppose that $k \geq l$, and we apply Lemma 2 repeatedly, decreasing the larger index by one and increasing the smaller by one, until we "meet in the middle". At this point we have an identity $\left\{H_{k}, H_{l}\right\}= \pm\left\{H_{m}, H_{n}\right\}$ where $m=n$ if $k$ and $l$ have the same parity, while $m=n+1$ if they have opposite parity. In the first case we get $\left\{H_{k}, H_{l}\right\}=0$ by the anti-symmetry of Poisson Brackets, and in the second case $\left\{H_{k}, H_{l}\right\}=0$ by e) of Lemma 1.

This finishes our partial proof of the NLS Hierarchy Theorem; we will complete the proof later.

\section{ZS-AKNS Direct Scattering Theory}

1. Statements of Results. For each potential $u$ in our phase space $\mathcal{S}\left(\mathbf{R}, \mathcal{T}^{\perp}\right)$ we would like to define scattering data, by which we will mean a measure of the asymptotic behavior of solutions of the parallel transport equation, $\psi_{x}=A(u, \lambda) \psi=$ $(\mathbf{a} \lambda+u) \psi$, for $x$ near $\pm \infty$. Of course, to have a useful Inverse Scattering Method, the scattering data for $u$ must be such that it allows us to recover $u$. On the other hand, it is preferable to make the scattering data as simple as possible, so it should be "just enough" to recover $u$. Direct Scattering Theory refers to this search for such good minimal scattering data and for the explicit determination of the image of the Direct Scattering Transform (the map from $u \in \mathcal{S}\left(\mathbf{R}, \mathcal{T}^{\perp}\right)$ to the scattering data of $u$ ). Identifying this image precisely is of course essential for a rigorous definition of the Inverse Scattering Transform that recovers $u$ from its scattering data. 
It turns out that, in discussing the asymptotic behavior of solutions $\psi$ of the parallel transport equation near infinity, it is more convenient to deal not with $\psi$ itself, but rather with the related function $\phi=\psi(x) e^{-\mathbf{a} \lambda x}$, which satisfies a slightly modified equation.

Proposition 1. If $\psi$ and $\phi$ are maps of $\mathbf{R}$ into $\mathbf{S L}(2, \mathbf{C})$ that are related by $\phi(x)=$ $\psi(x) e^{-\mathbf{a} \lambda x}$, then $\psi$ satisfies the parallel transport equation, $\psi_{x}=(\mathbf{a} \lambda+u) \psi$, if and only if $\phi$ satisfies what we shall call the "modified parallel transport equation", $\phi_{x}=[\mathbf{a} \lambda, \phi]+u \phi$.

Proof. Clearly $\phi_{x}=\psi_{x} e^{-\mathbf{a} \lambda x}-\psi e^{-\mathbf{a} \lambda x} \mathbf{a} \lambda=(\mathbf{a} \lambda+u) \psi e^{-\mathbf{a} \lambda x}-\phi \mathbf{a} \lambda$, and the result follows.

Definition. For $u$ in $\mathcal{S}\left(\mathbf{R}, \mathcal{T}^{\perp}\right)$, we will call $m^{u}(x, \lambda)$ a normalized eigenfunction of $u$ with eigenvalue $\lambda$ if it satisfies the modified parallel transport equation, $m_{x}^{u}=$ $\left[\mathbf{a} \lambda, m^{u}\right]+u m^{u}$, and if in addition:

1) $\lim _{x \rightarrow-\infty} m^{u}(x, \lambda)=I$.

2) $\sup _{x \in \mathbf{R}}\left\|m^{u}(x, \lambda)\right\|<\infty$.

It is these normalized eigenfunctions $m^{u}$ that will play the rôle of scattering data in this theory; they are analogous to the Jost solutions of the Schrödinger equation in the KdV theory. Note that condition 2) just means that each matrix element of $m^{u}(x, \lambda)$ is a bounded function of $x$.

A complete theory of normalized eigenfunctions will be found in [BC1]. We will next state the basic results proved there as three theorems, Theorem A, Theorem $\mathrm{B}$, and Theorem $\mathrm{C}$, reformulating things somewhat so as to make the statements better adapted to the Terng-Uhlenbeck version of inverse scattering theory that we will explain later. Then we will sketch the proofs of these results, leaving it to the interested reader to fill in many of the details from the original paper of Beals and Coifman.

We will denote $\mathcal{S}\left(\mathbf{R}, \mathcal{T}^{\perp}\right)$ by $P$ in what follows.

Theorem A. For each $u$ in $P$ there is a unique normalized eigenfunction $m^{u}(x, \lambda)$ for $u$ with eigenvalue $\lambda$, except for $\lambda$ in $\mathbf{R} \cup D^{u}$, where $D^{u}$ is a bounded, discrete subset of $\mathbf{C} \backslash \mathbf{R}$. Moreover, as a function of $\lambda$, for each fixed $x$ in $\mathbf{R}, m^{u}(x, \lambda)$ is meromorphic in $\mathbf{C} \backslash \mathbf{R}$ with poles at the points of $D^{u}$.

Note that a matrix-valued function of a complex variable is said to be holomorphic (resp., meromorphic) in a region $O$ if each of its matrix elements is holomorphic (resp., meromorphic) in $O$, and a pole of such a function is a pole of any of its matrix elements.

Definition. An element $u$ of $P$ will be called a regular potential if $D^{u}$ is a finite set and if, for all real $x$, the function $m^{u}(x, \lambda)$ with $\lambda$ in the upper half-plane $\mathbf{C}_{+}$has smooth boundary values $m_{+}^{u}(x, r)$ on the real axis, and similarly $m^{u}(x, \lambda)$ with $\lambda$ in the lower half-plane $\mathbf{C}_{-}$has smooth boundary values $m_{-}^{u}(x, r)$. We will denote the set of regular potentials by $P_{0}$.

Theorem B. The space $P_{0}$ of regular potentials is open and dense in the space $P=\mathcal{S}\left(\mathbf{R}, \mathcal{T}^{\perp}\right)$ of all potentials. 
It is an essential fact that the normalized eigenfunctions $m^{u}(x, \lambda)$ have asymptotic expansions as $|\lambda|$ tends to infinity. Since the precise nature of these expansions will be important, we will give the relevant definitions in some detail.

A matrix-valued function $f(\lambda)$ defined for complex $\lambda$ with $|\lambda|$ sufficiently large is said to have an asymptotic expansion at infinity if there exists a sequence of matrices $f_{n}$ so that $f(\lambda)-\sum_{j=0}^{k} f_{j} \lambda^{-j}=o\left(|\lambda|^{-k}\right)$. It is easy to see inductively that the $f_{n}$ are uniquely determined, and we write $f \sim \sum_{j} f_{j} \lambda^{-j}$.

Now suppose that we have matrix-valued functions $f(x, \lambda)$, defined for all $x$ in $\mathbf{R}$ and all $\lambda$ in $\mathbf{C}$ with $|\lambda|$ sufficiently large. Suppose that we have matrixvalued functions $f_{n}(x)$ such that for each $x, f(x, \lambda) \sim \sum_{j} f_{j}(x) \lambda^{-j}$. We will write $f \sim_{R} \sum_{j} f_{j} \lambda^{-j}$ if this asymptotic expansion holds uniformly in $x$, i.e., if

$$
\sup _{x}\left\|f(x, \lambda)-\sum_{j=0}^{k} f_{j}(x) \lambda^{-j}\right\|=o\left(|\lambda|^{-k}\right) .
$$

It is easy to explain the importance of the uniformity. Suppose $f$ and the $f_{n}$ are differentiable functions of $x$. Then the uniformity gives

$$
\frac{f(x+\Delta x, \lambda)-f(x, \lambda)}{\Delta x}-\sum_{j=0}^{k} \frac{f_{j}(x+\Delta x)-f_{j}(x)}{\Delta x} \lambda^{-j}=o\left(|\lambda|^{-k}\right),
$$

and letting $\Delta x$ approach zero gives $\frac{\partial f}{\partial x} \sim_{R} \sum_{j} f_{j}^{\prime} \lambda^{-j}$; i.e., we can differentiate such an asymptotic relation "term by term".

Theorem C. For $u$ in $P_{0}$, the normalized eigenfunctions $m^{u}(x, \lambda)$ have an asymptotic expansion as $\lambda$ tends to infinity, $m^{u} \sim_{R} \sum_{j} m_{j}^{u} \lambda^{-j}$. In fact the $m_{j}^{u}$ are uniquely determined inductively by the condition $\left[\mathbf{a}, m_{j+1}^{u}(x)\right]=\frac{d}{d x} m_{j}^{u}(x)-$ $u(x) m_{j}^{u}(x)$.

The normalized eigenfunctions, $m^{u}(x, \lambda)$, satisfy a simple relation, referred to as the "reality condition" that follows as an easy consequence of the fact that $u(x)$ takes its values in $\mathbf{s u}(2)$.

Proposition 2. If $u \in P_{0}$, then the normalized eigenfunctions $m^{u}$ satisfy the relation $m^{u}(x, \bar{\lambda})^{*} m^{u}(x, \lambda)=I$.

So, passing to the limit as $\lambda \in \mathbf{C}_{+}$approaches $r \in \mathbf{R}$,

Corollary. $m_{-}^{u}(x, r)^{*} m_{+}^{u}(x, r)=I$.

We will need one more property of the $m^{u}$ (or rather of their boundary values, $\left.m_{ \pm}^{u}\right)$.

Proposition 3. Let $u \in P_{0}$ and $x \in \mathbf{R}$, and let $m_{+}^{u}(x, r)=g(x, r) h(x, r)$ be the canonical decomposition of $m_{+}^{u}(x, r)$ into the product of a unitary matrix $g(x, r)$ and an upper-triangular matrix $h(x, r)$. Then $h(x, r)-I$ is of Schwartz class in $r$.

2. Outline of Proofs. As was the case for the scattering theory for the Schrödinger operator, it is a lot easier to see what is happening for the special case of potentials with compact support. It turns out for example that all such potentials are regular. Below we will give most of the details of the proofs of Theorems A, B, and $\mathrm{C}$ for the $2 \times 2$ case when $u$ has compact support. 
[In [BC1], Beals and Coifman consider first the case of compactly supported potentials, followed by the case of "small potentials", i.e., those with $L^{1}$ norm less than 1. For the latter, it turns out that existence and uniqueness of the $m^{u}$ can be proved easily using the Banach Contraction Principle, and moreover it follows that $D^{u}$ is empty. The case of regular potentials (called "generic" in [BC1]) is then handled by a limiting argument. Beals and Coifman actually consider the general $n \times n$ case and do not assume that $u$ is necessarily skew-adjoint. This latter generality adds substantial extra complexity to the argument.]

In any interval $[a, b]$ in which $u$ vanishes identically, the modified parallel transport equation reduces to the Lax Equation $\phi_{x}=[\mathbf{a} \lambda, \phi]$, so choosing an arbitrary $x_{0}$ in $[a, b]$, the solution is $\phi(x)=e^{\mathbf{a} \lambda\left(x-x_{0}\right)} \phi\left(x_{0}\right) e^{-\mathbf{a} \lambda\left(x-x_{0}\right)}$, or $\phi(x)=e^{\mathbf{a} \lambda x} s e^{-\mathbf{a} \lambda x}$, where we define $s=e^{-\mathbf{a} \lambda x_{0}} \phi\left(x_{0}\right) e^{\mathbf{a} \lambda x_{0}}$. This proves:

Proposition 4. Suppose $u$ in $P$ has compact support, say $u(x)=0$ for $|x| \geq M$. Then for each complex number $\lambda$ there is a unique solution $\phi^{u}(x, \lambda)$ of the modified parallel transport equation with $\phi^{u}(x, \lambda)=I$ for $x \leq-M$. Moreover, for $x \geq M$, $\phi^{u}$ has the form $\phi^{u}(x, \lambda)=e^{\mathbf{a} \lambda x} s^{u}(\lambda) e^{-\mathbf{a} \lambda x}$ (where $\left.s^{u}(\lambda)=e^{-\mathbf{a} \lambda M} \phi^{u}(M, \lambda) e^{\mathbf{a} \lambda M}\right)$, and for each real $x, \lambda \mapsto \phi^{u}(x, \lambda)$ is an entire function (i.e., holomorphic in all of C).

The fact that $\phi^{u}$ is holomorphic in $\lambda$ is a consequence of the more general principle that if an ODE depends analytically on a parameter $\lambda$, then the solution of the equation with some fixed initial condition is analytic in $\lambda$. (In this case the initial value condition is $\phi^{u}(-M, \lambda)=I$.)

Definition. We will denote the matrix elements of $s^{u}(\lambda)$ by $s_{i j}^{u}(\lambda)$, and we define $D^{u}$ to be the set of all $\lambda$ in the upper half-plane that are zeroes of $s_{11}^{u}$ union the set of all $\lambda$ in the lower half-plane that are zeroes of $s_{22}^{u}$.

Remark. It can be shown that the holomorphic functions $s_{12}^{u}$ and $s_{21}^{u}$ are not identically zero, so that $D^{u}$ is a discrete set. In fact (cf. [BC1], section 4), $D^{u}$ is finite, and neither $s_{11}^{u}$ nor $s_{22}^{u}$ has any zeroes on the real axis.

Proposition 5. Suppose $u$ in $P$ has compact support. For each $\lambda \in \mathbf{C} \backslash\left(\mathbf{R} \cup D^{u}\right)$ there is a unique normalized eigenfunction $m^{u}(x, \lambda)$. For every $x$ in $\mathbf{R}, m^{u}(x, \lambda)$ is a meromorphic function of $\lambda$ for $\lambda$ in $\mathbf{C} \backslash \mathbf{R}$, with poles at the points of $D^{u}$. Finally, the restriction of $m^{u}(x, \lambda)$ to each half-plane has a smooth extension to the real axis.

Proof. Since $\phi^{u}(x, \lambda)$ is invertible, it is no loss of generality to assume that a normalized eigenfunction has the form $m^{u}(x, \lambda)=\phi^{u}(x, \lambda) \chi^{u}(x, \lambda)$. Then $\left[\mathbf{a} \lambda, m^{u}\right]+$ $u m^{u}=m_{x}^{u}=\phi_{x}^{u} \chi^{u}+\phi \chi_{x}^{u}$, which simplifies to the same Lax Equation as before, namely $\chi_{x}^{u}=\left[\mathbf{a} \lambda, \chi^{u}\right]$, but now valid on the whole of $\mathbf{R}$, and it follows that $\chi^{u}(x, \lambda)=e^{\mathbf{a} \lambda x} \chi^{u}(\lambda) e^{-\mathbf{a} \lambda x}$, and hence $m^{u}(x, \lambda)=\phi^{u}(x, \lambda) e^{\mathbf{a} \lambda x} \chi^{u}(\lambda) e^{-\mathbf{a} \lambda x}$.

Then, by Proposition 4 , for $x \leq-M, m^{u}(x, \lambda)=e^{\mathbf{a} \lambda x} \chi^{u}(\lambda) e^{-\mathbf{a} \lambda x}$, while for $x \geq M, m^{u}(x, \lambda)=e^{\mathbf{a} \lambda x} s^{u}(\lambda) \chi^{u}(\lambda) e^{-\mathbf{a} \lambda x}$.

Let us write $\chi_{i j}^{u}(\lambda)$ for the matrix elements of $\chi^{u}(\lambda)$ and try to determine them individually so that Conditions 1 ) and 2) of the definition of generalized eigenfunctions will be satisfied for the resulting $m^{u}(x, \lambda)$.

Note that since conjugating $\chi^{u}(\lambda)$ by a diagonal matrix does not change its diagonal entries, the diagonal elements of $m^{u}(x, \lambda)$ are just $\chi_{11}^{u}(\lambda)$ and $\chi_{22}^{u}(\lambda)$ for $x \leq-M$. Since Condition 1) requires that $m^{u}(x, \lambda)$ converge to the identity 
matrix as $x$ approaches $-\infty$, it follows that we must take $\chi_{11}^{u}(\lambda)=\chi_{22}^{u}(\lambda)=1$, and conversely with this choice Condition 1) is clearly satisfied.

On the other hand, an easy calculation shows that the off-diagonal elements, $m_{12}^{u}(x, \lambda)$ and $m_{21}^{u}(x, \lambda)$, are given respectively by $e^{-2 i \lambda x} \chi_{12}^{u}(\lambda)$ and $e^{2 i \lambda x} \chi_{21}^{u}(\lambda)$, when $x \leq-M$. If $\lambda=\sigma+i \tau, m_{12}^{u}(x, \lambda)=e^{-2 i \sigma x} e^{2 \tau x} \chi_{12}^{u}(\lambda)$, and $m_{21}^{u}(x, \lambda)=$ $e^{2 i \sigma x} e^{-2 \tau x} \chi_{21}^{u}(\lambda)$. Since Condition 2) requires that these remain bounded when $x$ approaches $-\infty$, it follows that when $\lambda$ is in the lower half-plane (i.e., $\tau<0$ ), then $\chi_{12}^{u}(\lambda)=0$, and similarly, $\chi_{21}^{u}(\lambda)=0$ for $\lambda$ in the upper half-plane.

Next, take $x>M$, so that $m^{u}(x, \lambda)=e^{\mathbf{a} \lambda x} s^{u}(\lambda) \chi^{u}(\lambda) e^{-\mathbf{a} \lambda x}$. Then another easy computation shows that if $\lambda$ is in the upper half-plane, then $m_{12}^{u}(x, \lambda)=$ $e^{-2 i \lambda x}\left(s_{11}^{u}(\lambda) \chi_{12}^{u}(\lambda)+s_{11}^{u}(\lambda)\right)$, while $m_{12}^{u}(x, \lambda)=0$. Since $m_{11}^{u}(x, \lambda)=s_{11}^{u}(\lambda)$ and $m_{22}^{u}(x, \lambda)=s_{22}^{u}(\lambda)$ are independent of $x$, the condition for $m^{u}(\lambda)$ to remain bounded when $x$ approaches $+\infty$ is just $s_{11}^{u}(\lambda) \chi_{12}^{u}(\lambda)+s_{12}^{u}(\lambda)=0$, and this uniquely determines $\chi_{12}^{u}(\lambda)$, namely $\chi_{12}^{u}(\lambda)=-s_{12}^{u}(\lambda) / s_{11}^{u}(\lambda)$. So for $\lambda$ in the upper halfplane $\chi^{u}(\lambda)=\left(\begin{array}{cc}1 & -s_{12}^{u}(\lambda) / s_{11}^{u}(\lambda) \\ 0 & 1\end{array}\right)$ is the unique choice of $\chi^{u}$ satisfying Conditions 1) and 2). A similar computation shows that for $\lambda$ in the lower half-plane $\chi^{u}(\lambda)=\left(\begin{array}{cc}1 & 0 \\ s_{21}^{u}(\lambda) / s_{22}^{u}(\lambda) & 1\end{array}\right)$. All conclusions of the proposition follow from these explicit formulas and the fact that $s_{11}$ and $s_{22}$ have no zeroes on the real axis.

Lemma. If $\psi_{x}=A \psi$ and $\phi_{x}=-\phi A$, then $\phi \psi$ is constant.

Proof. $(\phi \psi)_{x}=\phi_{x} \psi+\phi \psi_{x}=0$.

We can now prove Proposition 2.

Proof. It will suffice to prove that $m^{u}(x, \bar{\lambda})^{*} m^{u}(x, \lambda)$ is constant, since we know that as $x$ approaches $-\infty$ the product converges to $I$. If we define $\psi(x, \lambda)=$ $m^{u}(x, \lambda) e^{\mathbf{a} \lambda x}$, then $\psi(x, \bar{\lambda})^{*}=e^{-\mathbf{a} \lambda x} m^{u}(x, \bar{\lambda})$, and therefore $m^{u}(x, \bar{\lambda})^{*} m^{u}(x, \lambda)=$ $\psi(x, \bar{\lambda})^{*} \psi^{u}(x, \lambda)$ and it will suffice to prove that $\psi(x, \bar{\lambda})^{*} \psi^{u}(x, \lambda)$ is constant. By Proposition $1, \psi_{x}(x, \lambda)=(\mathbf{a} \lambda+u) \psi(x, \lambda)$. Since $u^{*}=-u$ and $(\mathbf{a} \bar{\lambda})^{*}=-\mathbf{a} \lambda$, $\psi_{x}(x, \bar{\lambda})^{*}=\psi(x, \bar{\lambda})^{*}(\mathbf{a} \bar{\lambda}+u)^{*}=-\psi(x, \bar{\lambda})^{*}(\mathbf{a} \lambda+u)$, and the preceding lemma completes the proof.

Our Theorem $\mathrm{C}$ is just Theorem 6.1, page 58, of [BC1]. While the proof is not difficult, neither is it particularly illuminating, and we will not repeat it here. Similarly, our Proposition 3 follows from Theorem $\mathrm{E}^{\prime}$, page 44, of [BC1].

This completes our discussion of the proofs of Theorems A, B, C, and Propositions 2 and 3. In the remainder of this section we will see how these results can be used to complete the proof of the NLS Hierarchy Theorem.

Since $m^{u}(x, \lambda)^{-1}=m^{u}(x, \bar{\lambda})^{*}$, it follows that $m^{u}(x, \lambda) \mathbf{a}\left(m^{u}(x, \lambda)\right)^{-1}$ has an asymptotic expansion.

Definition. We denote the function $m^{u}(x, \lambda) \mathbf{a}\left(m^{u}(x, \lambda)\right)^{-1}$ by $Q^{u}(x, \lambda)$.

So by the preceding remark,

Corollary. $Q^{u}(x, \lambda)$ has an asymptotic expansion $Q^{u} \sim_{R} \sum_{j=0}^{\infty} Q_{j}^{u} \lambda^{-j}$, with $Q_{0}^{u}=$ a; hence also $Q_{x}^{u} \sim_{R} \sum_{j=0}^{\infty}\left(Q_{j}^{u}\right)_{x} \lambda^{j}$. 
Lemma. If we define $\psi(x, \lambda)=m^{u}(x, \lambda) e^{\mathbf{a} \lambda x}$ then $Q^{u}(x, \lambda)=\psi \mathbf{a} \psi^{-1}$.

Proof. Immediate from the fact that all diagonal matrices commute.

Since $\left(\psi \mathbf{a} \psi^{-1}\right)_{x}=\psi_{x} \mathbf{a} \psi^{-1}+\psi \mathbf{a}\left(\psi^{-1}\right)_{x}$, by Proposition 1, $\psi_{x}=(\mathbf{a} \lambda+u) \psi$. Also, from $\psi \psi^{-1}=I$ we get $\psi_{x} \psi^{-1}+\psi\left(\psi^{-1}\right)_{x}=0$. Combining all these facts gives $\left(\psi \mathbf{a} \psi^{-1}\right)_{x}=\left[\mathbf{a} \lambda+u, \psi \mathbf{a} \psi^{-1}\right]$, and hence, by the lemma, $Q_{x}^{u}(x, \lambda)=[\mathbf{a} \lambda+$ $\left.u, Q^{u}(x, \lambda)\right]$. If we insert in this identity the asymptotic expansion $Q^{u} \sim_{R}$ $\sum_{j=0}^{\infty} Q_{j}^{u} \lambda^{j}$, we find a second asymptotic expansion for $Q_{x}^{u}(x, \lambda)$, in addition to the one from the above corollary, namely $Q_{x}^{u} \sim_{R} \sum_{j}\left(\left[a, Q_{j+1}^{u}\right]+\left[u, Q_{j}^{u}\right]\right) \lambda^{j}$. Therefore, by uniqueness of asymptotic expansions we have proved:

Proposition 6. The recursion relation $\left(Q_{j}^{u}\right)_{x}=\left[a, Q_{j+1}^{u}\right]+\left[u, Q_{j}^{u}\right]$ is satisfied by the coefficients $Q_{j}^{u}$ of the asymptotic expansion of $Q^{u}(x, \lambda)$, and hence they are identical with the functions $Q_{j}(u): \mathbf{R} \rightarrow \mathbf{s u}(2)$ defined in the NLS Hierarchy Theorem.

We are now finally in a position to complete the proof of the NLS Hierarchy Theorem.

Since $\mathbf{a}^{2}=I$, it follows that also $Q^{u}(x, \lambda)^{2}=\left(m^{u} \mathbf{a}\left(m^{u}\right)^{-1}\right)^{2}=I$, and hence $I \sim\left(\sum_{j=0}^{\infty} Q_{j}^{u} \lambda^{j}\right)^{2}$. Expanding and comparing coefficients of $\lambda^{-k}$, uniqueness of asymptotic expansions gives $\mathbf{a} Q_{k}(u)+Q_{k}(u) \mathbf{a}=-\sum_{j=1}^{k-1} Q_{j}(u) Q_{k-j}(u)$. Recall that we needed one fact to complete the proof of statements a) through d) of the NLS Hierarchy Theorem, namely that if $Q_{k}(u)=P_{k}(u)+T_{k}(u)$ is the decomposition of $Q_{k}(u)$ into its off-diagonal part and its diagonal part, then the matrix elements of $T_{k}(u)$ are polynomials in the matrix elements of $u$ and their derivatives. Moreover, we saw that we could assume inductively that this was true for the matrix elements of $Q_{j}(u)$ for $j<k$. But if $T_{k}=\left(\begin{array}{cc}t_{k} & 0 \\ 0 & -t_{j}\end{array}\right)$, then $\mathbf{a} Q_{k}(u)+Q_{k}(u) \mathbf{a}=-2 i T_{k}=\left(\begin{array}{cc}-2 i t_{k} & 0 \\ 0 & -2 i t_{j}\end{array}\right)$, and the desired result is now immediate from the inductive assumption.

The other statement of the NLS Hierarchy Theorem that remains to be proved is e).

Define a function $\tilde{F}^{u}(x, \lambda)=\operatorname{tr}\left(Q^{u}(x, \lambda) \mathbf{a}\right)$. Clearly $\tilde{F}^{u}(x, \lambda)$ has an asymptotic expansion, $\tilde{F}^{u} \sim_{R} \sum_{j} \tilde{F}_{j}^{u} \lambda^{-j}$, where $\tilde{F}_{j}^{u}=\operatorname{tr}\left(Q_{j}^{u} \mathbf{a}\right)$.

From what we have just seen, $\tilde{F}^{u}(x, \lambda)$ is Schwartz class in $x$, so we can define a map $F(u, \lambda)=\int_{-\infty}^{\infty} \tilde{F}^{u}(x, \lambda) d x=\int_{-\infty}^{\infty} \operatorname{tr}\left(Q^{u} \mathbf{a}\right) d x$, and $F(u, \lambda) \sim \sum_{j} F_{j}(u) \lambda^{-j}$ where $F_{j}(u)=\int_{-\infty}^{\infty} \tilde{F}_{j}^{u}(x) d x$.

If we consider $u \mapsto F(u, \lambda)$ as a function on $P$, then $\nabla F$ is a vector field on $P$, and $(\nabla F)_{u} \sim \sum_{j}\left(\nabla F_{j}\right)_{u} \lambda^{-j}$. We claim that statement e) of the NLS Hierarchy Theorem follows from the following proposition. (For a proof of which, see Proposition 2.4 of [Te2].)

Proposition 7. If $v$ in $P_{0}$ then

$$
\left.\frac{d}{d \epsilon}\right|_{\epsilon=0} F(u+\epsilon v, \lambda)=\int_{-\infty}^{\infty} \operatorname{tr}\left(\frac{d Q^{u}(x, \lambda)}{d \lambda} v(x) \mathbf{a}\right) d x .
$$

Indeed, expand both sides of the latter equality in asymptotic series in $\lambda$, and compare coefficients of $\lambda^{-j}$. Since $\frac{d Q^{u}(x, \lambda)}{d \lambda}=\sum_{j}-j Q_{j}^{u} \lambda^{-j-1}$, we find $\left(d F_{j}\right)_{u}(v)=$ 
$\int_{-\infty}^{\infty} \operatorname{tr}\left(-(j-1) Q_{j-1}(u)(x) v(x) \mathbf{a}\right) d x$. Recalling the definition of the inner product in $P$, we see $-\frac{1}{j-1}\left(\nabla F_{j}\right)_{u}$ is the projection of $Q_{j-1}(u)$ on $\mathcal{T}^{\perp}$, i.e., the off-diagonal part of $Q_{j-1}(u)$. So if we define $H_{j}(u)=-\frac{1}{j+1} \int_{-\infty}^{\infty} \operatorname{tr}\left(Q_{j+2}(u) \mathbf{a}\right) d x=-\frac{1}{j+1} F_{j+2}(u)$, then $\left(\nabla H_{j}\right)_{u}=-\frac{1}{j+1}\left(\nabla F_{j+2}\right)_{u}$ is the off-diagonal part of $Q_{j+1}(u)$, which is statement e) of the NLS Hierarchy Theorem.

\section{Loop Groups, Dressing Actions, and Inverse Scattering}

1. Secret Sources of Soliton Symmetries. This article is titled "The Symmetries of Solitons", and we have been hinting that many of the remarkable properties of soliton equations are closely related to the existence of large and non-obvious groups of symplectic automorphisms that act on the phase spaces of these Hamiltonian systems and leave the Hamiltonian function invariant. We are now finally in a position where we can describe these groups and their symplectic actions.

The groups themselves are so-called loop groups. While they have been around in various supporting rôles for much longer, in the past three decades they have been increasingly studied for their own sake and have attained a certain prominence. See for example [PrS].

Given any Lie group $\mathbf{G}$, we can define its associated loop group, $L(\mathbf{G})$, as the group of all maps (of some appropriate smoothness class) of $\mathbf{S}^{1}$ into $\mathbf{G}$, with pointwise composition. For our purposes we will always assume that $\mathbf{G}$ is a matrix group and the maps are smooth (i.e., infinitely differentiable).

The theory gets more interesting when we regard the loops in $\mathbf{G}$ as boundary values of functions that are holomorphic (or meromorphic) in the interior (or exterior) of the unit disk and take subgroups by restricting the analytic properties of these analytic extensions. That is, we concentrate on the analytic extensions rather than the boundary value.

Once we take this point of view, it is just as natural to pre-compose with a fixed linear fractional transformation mapping the real line to the unit circle, say $z \mapsto(1+i z) /(1-i z)$, so that elements of the loop groups become maps of $\mathbf{R}$ into $\mathbf{G}$ that are boundary values of certain analytic functions in the upper or lower half-plane, and this is the point of view we will adopt. Note that the above linear fractional transformation takes -1 in $\mathbf{S}^{1}$ to infinity, and for certain purposes it is important to know how the nature of the original map of $\mathbf{S}^{1}$ into $\mathbf{G}$ at -1 translates to properties of the transformed map of $\mathbf{R}$ into $\mathbf{G}$ at $\pm \infty$. A straightforward calculation gives the following answer:

Proposition 1. ([TU1], Proposition 7.7) Given $g: \mathbf{S}^{1} \rightarrow \mathbf{G L}(n, \mathbf{C})$, define $\Phi(g)$ : $\mathbf{R} \rightarrow \mathbf{G L}(n, \mathbf{C})$ by $\Phi(g)(r)=g\left(\frac{1+i r}{1-i r}\right)$. Then $:$

(i) $g$ is smooth if and only if $\Phi(g)$ is smooth and has asymptotic expansions at $+\infty$ and at $-\infty$ and these expansions agree.

(ii) $g-I$ is infinitely flat at $z=-1$ if and only if $\Phi(g)-I$ is of Schwartz class.

(iii) $g: \mathbf{C} \rightarrow \mathbf{G L}(n, \mathbf{C})$ satisfies the reality condition $g\left(\frac{1}{\bar{z}}\right)^{*} g(z)=I$ if and only if $\Phi(g)(\lambda)=g\left(\frac{1+i \lambda}{1-i \lambda}\right)$ satisfies $\Phi(g)(\bar{\lambda})^{*} \Phi(g)(\lambda)=I$.

The first, and most important, loop group we will need is called $\mathcal{D}_{-}$. The analytic properties of its elements are patterned after those proved to hold in the preceding section for the normalized eigenfunctions $m^{u}(x, \lambda)$ as functions of $\lambda$. 
Definition. We will denote by $\mathcal{D}_{-}$the group of all meromorphic maps $f: \mathbf{C} \backslash \mathbf{R} \rightarrow$ $\mathbf{G L}(n, \mathbf{C})$ having the following properties:

1) $f(\bar{\lambda}) * f(\lambda)=I$.

2) $f$ has an asymptotic expansion $f(\lambda) \sim I+f_{1} \lambda^{-1}+f_{2} \lambda^{-2}+\cdots$.

3) The set $D^{f}$ of poles of $f$ is finite.

4) $f$ restricted to the upper half-plane, $\mathbf{C}_{+}$, extends to a smooth function on the closure of the upper-half plane, and similarly for the lower half-plane. The boundary values are then maps $f_{ \pm}: \mathbf{R} \rightarrow \mathbf{G L}(n, \mathbf{C})$, and by 1$)$ they satisfy $f_{+}(r)^{*} f_{-}(r)=I$.

5) If $f_{+}(r)=g(r) h(r)$ is the factorization of $f_{+}(r)$ as the product of a unitary matrix $g(r)$ and an upper triangular $h(r)$, then $h-I$ is of Schwartz class.

Definition. We define a map, $\mathcal{F}_{\text {scat }}: P_{0} \rightarrow \mathcal{D}_{-}$, the Scattering Transform, by $\mathcal{F}_{\text {scat }}(u)(\lambda)=f^{u}(\lambda)=m^{u}(0, \lambda)$.

That $m^{u}(0, \lambda)$ is in fact an element of $\mathcal{D}_{-}$is a consequence of the definition of the set $P_{0}$ of regular potentials, and Theorems A and $\mathrm{C}$ and Propositions 2 and 3 of the preceding section. There is nothing special about 0 in the above definition. We could have equally well chosen any other fixed real number $x_{0}$ and used $m^{u}\left(x_{0}, \lambda\right)$ instead of $m^{u}(0, \lambda)$.

2. Terng-Uhlenbeck Factoring and the Dressing Action. There are three other loop groups that play an essential rôle in the definition of the Inverse Scattering Transform, $\mathcal{I F}$ scat , and we define these next.

Definition. We will denote by $\mathcal{G}_{+}$the loop group of all entire functions $h: \mathbf{C} \rightarrow$ $\mathbf{G L}(n, \mathbf{C})$, and by $\mathcal{H}_{+}$the abelian subgroup of $\mathcal{G}_{+}$consisting of all elements of the form $e^{\mathbf{a} P(\lambda)}$ where $P: \mathbf{C} \rightarrow \mathbf{C}$ is a polynomial in $\lambda$. Finally, we define $\mathcal{H}_{-}$to be the subgroup of $\mathcal{D}_{-}$consisting of those elements $f$ taking values $f(\lambda)$ in the diagonal subgroup of $\mathbf{G L}(n, \mathbf{C})$. For each $x$ in $\mathbf{R}$ we define $e_{\mathbf{a}}(x)$ in $\mathcal{H}_{+}$by $e_{\mathbf{a}}(x)(\lambda)=e^{\mathbf{a} \lambda x}$, and for each positive integer $j$ we define a one-parameter subgroup $e_{\mathbf{a}, j}$ of $\mathcal{H}_{+}$by $e_{\mathbf{a}, j}(t)=e^{\mathbf{a} \lambda^{j} t}$. (Note that $e_{\mathbf{a}}(x)=e_{\mathbf{a}, 1}(x)$.)

The following theorem is one of the basic results of [TU1]. As we shall see, it provides an alternative, group theoretic approach to ZS-AKNS Inverse Scattering Theory. (In fact, conversely, it can be proved using earlier approaches to ZS-AKNS Inverse Scattering Theory).

Terng-Uhlenbeck Factoring Theorem. ([TU1], 7.11 and 7.16) If $f \in \mathcal{D}_{-}$, then:

1) for any $h \in \mathcal{H}_{+}, h f^{-1}: \mathbf{C} \backslash\left(\mathbf{R} \cup D^{f}\right) \rightarrow \mathbf{G L}(n, \mathbf{C})$ can be factored uniquely in the form $h f^{-1}=M^{-1} E$, with $M$ in $\mathcal{D}_{-}$and $E$ in $\mathcal{G}_{+}$.

2) Taking $h=e_{\mathbf{a}, 1}(x)$ in 1) (i.e., $h(\lambda)=e^{\mathbf{a} \lambda x}$ ), we get a one-parameter family of such factorings, $e_{\mathbf{a}, 1}(x) f^{-1}=M^{-1}(x) E(x)$ and, writing $E_{x}$ for the derivative of $E$, it follows that $E_{x}=(\mathbf{a} \lambda+u) E$ for a unique, regular potential $u$ in $P_{0}$.

We note that in 1) uniqueness is easy and only existence of the decomposition needs proof. Indeed, uniqueness is equivalent to the statement that $\mathcal{D}_{-} \cap \mathcal{G}_{+}=I$, and this is immediate from Liouville's Theorem that bounded holomorphic functions are constant (recall that elements of $\mathcal{D}_{-}$converge to $I$ as $\lambda \rightarrow \infty$ ). The existence part of 1) follows from the two classical Birkhoff Decomposition Theorems, and statement 2) gives the dependence of this factorization on the parameter $x$. 
Definition. We define a left action of $\mathcal{H}_{+}$on $\mathcal{D}_{-}$, called the dressing action, and denoted by $(h, f) \mapsto h * f$. It is defined by $h * f=M$, where $M$ is given by the factoring of $h f^{-1}$ in 1) of the previous theorem.

Of course we must check that $\left(h_{1} h_{2}\right) * f=h_{1} *\left(h_{2} * f\right)$, but this is easy. Suppose $h_{2} f^{-1}=M_{2}^{-1} E_{2}$, i.e., $h_{2} * f=M_{2}$, and use the factoring theorem again to write $h_{1} M_{2}^{-1}$ as a product, $h_{1} M_{2}^{-1}=M_{1}^{-1} E_{1}$, i.e., $h_{1} * M_{2}=M_{1}$. Then $\left(h_{1} h_{2}\right) f^{-1}=$ $h_{1}\left(h_{2} f^{-1}\right)=h_{1} M_{2}^{-1} E_{2}=M_{1}^{-1} E_{1} E_{2}$, so $\left(h_{1} h_{2}\right) * f=M_{1}=h_{1} * M_{2}=h_{1} *\left(h_{2} * f\right)$.

Now that we have an action of $\mathcal{H}_{+}$on $\mathcal{D}_{-}$, it follows that every one-parameter subgroup of $\mathcal{H}_{+}$defines a flow on $\mathcal{D}_{-}$. In particular the one-parameter subgroups $e_{\mathbf{a}, j}$ define an important sequence of flows on $\mathcal{D}_{-}$.

Definition. For each positive integer $j$ we define a flow on $\mathcal{D}_{-}$, called the $j$-th flow, by $(t, f) \mapsto e_{\mathbf{a}, j}(t) * f$.

Of course, since $\mathcal{H}_{+}$is an abelian group and all the $e_{\mathbf{a}, j}$ are one-parameter subgroups of $\mathcal{H}_{+}$, it follows that this sequence of flows all mutually commute.

3. The Inverse Scattering Transform. We are now in a position to define the Inverse Scattering Transform.

Definition. We define a map $\mathcal{I} F_{\text {scat }}: \mathcal{D}_{-} \rightarrow P_{0}$, called Inverse Scattering Transform, by associating to $f$ in $\mathcal{D}_{-}$the regular potential $u=\mathcal{I} F_{\text {scat }}(f)$ in $P_{0}$ given by 2) of the Terng-Uhlenbeck Factoring Theorem. That is, if we define $\psi(x, \lambda)=$ $\left(e_{\mathbf{a}}(x) * f\right)(\lambda) e^{\mathbf{a} \lambda x}$, then $u$ is characterized by the fact that $\psi$ satisfies the parallel transport equation with potential $u, \psi_{x}=(\mathbf{a} \lambda+u) \psi$.

Theorem D. The maps $\mathcal{F}_{\text {scat }}: P_{0} \rightarrow \mathcal{D}_{-}$and $\mathcal{I F}_{\text {scat }}: \mathcal{D}_{-} \rightarrow P_{0}$ satisfy:

a) $\mathcal{I} F_{\text {scat }} \circ \mathcal{F}_{\text {scat }}=$ identity.

b) $\mathcal{F}_{\text {scat }} \circ \mathcal{I} F_{\text {scat }}(f) \in f \mathcal{H}_{-}$.

Thus, the map $P_{0} \rightarrow \mathcal{D}_{-} \rightarrow \mathcal{D}_{-} / \mathcal{H}_{-}$that is the composition of $\mathcal{F}_{\text {scat }}$ and the natural projection of $\mathcal{D}_{-}$on $\mathcal{D}_{-} / \mathcal{H}_{-}$is a bijection.

Recall that in the NLS-Hierarchy Theorem we defined a sequence of flows on $P_{0}$, the $j$-th of which we also called the " $j$-th flow". As you probably suspect:

Theorem E. ([TU1], Theorem 8.1) The transforms $\mathcal{F}_{\text {scat }}: P_{0} \rightarrow \mathcal{D}_{-}$and $\mathcal{I F}_{\text {scat }}:$ $\mathcal{D}_{-} \rightarrow P_{0}$ are equivariant with respect to the $j$-th flow on $\mathcal{D}_{-}$and the $j$-th flow on $P_{0}$. In particular if $u(t)$ in $P_{0}$ is a solution of the $j$-th flow, then $\mathcal{F}_{\text {scat }}(u(t))=$ $e_{\mathbf{a}, j}(t) * \mathcal{F}_{\text {scat }}(u(0))$.

Corollary. The following algorithm finds the solution $u(x, t)$ for the $j$-th flow in $P_{0}$ with initial condition $u(0)=u(x, 0)$ :

1) Compute the parallel translation operator $\psi(x, 0, \lambda)$ having the correct asymptotic behavior. That is, solve the following linear ODE problem:

a) $\psi_{x}(x, 0, \lambda)=(\mathbf{a} \lambda+u(x, 0)) \psi(x, 0, \lambda)$.

b) $\lim _{x \rightarrow-\infty} \psi(x, 0, \lambda) e^{-\mathbf{a} \lambda x}=I$.

c) $\psi(x, 0, \lambda) e^{-\mathbf{a} \lambda x}$ is bounded.

2) Define $f$ in $\mathcal{D}_{-}$by $f(\lambda)=\psi(0,0, \lambda)$.

3) Factor $e_{a, j}(t) e_{a, 1}(x) f^{-1}$ as $M(x, t)^{-1} E(x, t)$ with $M(x, t) \in \mathcal{D}_{-}$and $E(x, t) \in$ $\mathcal{G}_{+}$.

4) Then, putting $\psi(x, t, \lambda)=M(x, t)(\lambda) e^{\mathbf{a} \lambda x+\lambda^{j} t}$, $u(x, t)=\psi_{x}(x, t, \lambda) \psi^{-1}(x, t, \lambda)-\mathbf{a} \lambda$. (The RHS is independent of $\lambda$.) 
Proof. This just says that $u(t)=\mathcal{I F}_{\text {scat }}\left(e_{\mathbf{a}, j}(t) * \mathcal{F}_{\text {scat }}(u(0))\right)$.

4. ZS-AKNS Scattering Coordinates. An important ingredient of the KdV Inverse Scattering Method, based on the Schrödinger operator, was that the "coordinates" of the scattering data evolved by a linear ODE with constant coefficients, and so this evolution could be solved explicitly. Recall that this allowed us to derive an explicit formula for the KdV multi-solitons. Such scattering coordinates (or "action-angle variables") also exist for the ZS-AKNS Hierarchy, and even for the more general $n \times n$ systems, but the story is somewhat more complicated in this case, and we will only outline the theory here and refer to $[\mathrm{ZS}]$ and $[\mathrm{BS}]$ for more complete descriptions.

Another advantage of the loop group approach is that it permits us to factor the scattering data into discrete and continuous parts. To a certain extent this allows us to discuss separately the scattering coordinates and evolution of each part.

Definition. We define two subgroups, $\mathcal{D}_{-}^{\text {disc }}$ and $\mathcal{D}_{-}^{\text {cont }}$, of $\mathcal{D}_{-}$, by

$$
\begin{aligned}
& \mathcal{D}_{-}^{\text {cont }}=\left\{f \in \mathcal{D}_{-} \mid f \text { is holomorphic in } \mathbf{C} \backslash \mathbf{R}\right\}, \text { and } \\
& \mathcal{D}_{-}^{\text {disc }}=\left\{f \in \mathcal{D}_{-} \mid f \text { is meromorphic in } \mathbf{C}\right\} .
\end{aligned}
$$

Remark. Since elements of $\mathcal{D}_{-}$approach $I$ at infinity, it follows that any $f$ in $\mathcal{D}_{-}^{\text {disc }}$ is actually meromorphic on the whole Riemann sphere, and hence a rational function of the form $f_{i j}(\lambda)=P_{i j}(\lambda) / Q_{i j}(\lambda)$, where the polynomial maps $P_{i j}$ and $Q_{i j}$ have the same degrees for a given diagonal entry, and $Q_{i j}$ has larger degree for an offdiagonal entry. For this reason, $\mathcal{D}_{-}^{\text {disc }}$ is also referred to as the rational subgroup of $\mathcal{D}_{-}$. Also, since $f$ satisfies the reality condition, $f \overline{(\lambda}(\lambda) f(\lambda)=I$ and is holomorphic on the real line, it follows that for $r$ in $\mathbf{R}, f(r)^{*} f(r)=I$ (i.e., $f$ is unitary on $\mathbf{R}$ ), and the boundary values $f_{+}$of $f$ from $\mathbf{C}_{+}$and $f_{-}$from $\mathbf{C}_{-}$are equal, so that the "jump", $v^{f}(r)=f_{-}^{-1}(r) f_{+}(r)$, is the identity.

Theorem F. ([TU1], Theorem 7.5) Every $f$ in $\mathcal{D}_{-}$can be factored uniquely as a product $f=h g$ where $h \in \mathcal{D}_{-}^{\text {cont }}$ and $g \in \mathcal{D}_{-}^{\text {cont }}$. In fact the multiplication map $\mathcal{D}_{-}^{\text {cont }} \times \mathcal{D}_{-}^{\text {disc }} \rightarrow \mathcal{D}_{-}$is a diffeomorphism.

Proof. This is an immediate consequence of Proposition 1 of the previous section and the following classical theorem of G. D. Birkhoff.

Birkhoff Decomposition Theorem. ([PrS], Theorem 8.1.1) Let $L(\mathbf{G L}(n, \mathbf{C})$ ) denote the loop group of all smooth maps of $\mathbf{S}^{1}$ into $\mathbf{G L}(n, \mathbf{C}), \Omega \mathbf{U}(n)$ the subgroup of all smooth maps $g$ of $\mathbf{S}^{1}$ into $\mathbf{U}(n)$ such that $g(-1)=I$, and $L^{+}(\mathbf{G L}(n, \mathbf{C}))$ the subgroup of $L(\mathbf{G L}(n, \mathbf{C}))$ consisting of all $g$ that are the boundary values of holomorphic maps of the open unit disk into $\mathbf{G} \mathbf{L}(n, \mathbf{C})$. Then any $f$ in $L(\mathbf{G L}(n, \mathbf{C}))$ can be factored uniquely as a product $f=g h$ where $g \in L^{+}(\mathbf{G L}(n, \mathbf{C}))$ and $h \in \Omega \mathbf{U}(n)$. In fact the multiplication map $L^{+}(\mathbf{G L}(n, \mathbf{C})) \times \Omega \mathbf{U}(n) \rightarrow L(\mathbf{G L}(n, \mathbf{C}))$ is a diffeomorphism.

Definition. Given $z \in \mathbf{C}$ and an orthogonal projection $\pi$ in $\mathbf{G L}(n, \mathbf{C})$, we define $g_{z, \pi}$ in $\mathcal{D}_{-}^{\text {disc }}$ by $g_{z, \pi}(\lambda)=I+\frac{z-\bar{z}}{\lambda-z} \pi$. 
Theorem G. (Uhlenbeck [U1]) The elements $g_{z, \pi}$ for $z \in \mathbf{C} \backslash \mathbf{R}$ generate the group $\mathcal{D}_{-}^{\text {disc }}$.

It follows easily from Theorem $\mathrm{G}$ and the Bianchi Permutability Formula ([TU1], Theorem 10.13) that at each simple pole $z$ of an element of $\mathcal{D}_{-}^{\text {disc }}$ we can define a "residue", which is just the image of a certain orthogonal projection, $\pi$. To be precise:

Theorem H. If $f \in \mathcal{D}_{-}$and $z$ is a simple pole of $f$, then there exists a unique orthogonal projection $\pi$ such that $f g_{z, \pi}^{-1}$ is holomorphic at $z$.

The set of $f$ in $\mathcal{D}_{-}$for which all the poles are simple is open and dense, and it is for these $f$ that we will define "scattering coordinates", $S^{f}$.

Definition. Given $f$ in $\mathcal{D}_{-}$with only simple poles, the scattering coordinates of $f$, $S^{f}$ consist of the following data:

a) The set $D^{f}=\left\{z_{1}, \ldots, z_{N}\right\}$ of poles of $f$.

b) For each $z$ in $D^{f}$, the "residue" of $f$ at $z$, i.e., the image $V_{z}^{f}$ of the unique orthogonal projection, $\pi=\pi_{z}^{f}$ such that $f g_{z, \pi}^{-1}$ is holomorphic at $z$.

c) The jump function of $f$, i.e., the map $v^{f}: \mathbf{R} \rightarrow \mathbf{G L}(n, \mathbf{C})$ defined by $v^{f}(r)=$ $f_{-}^{-1}(r) f_{+}(r)$.

The following theorem describes the evolution of the scattering coordinates $S^{f}$.

Theorem I. ([TU1]) If $f(t) \in \mathcal{D}_{-}$evolves by the $j$-th flow and $f(0)$ has only simple poles, then $S^{f(t)}$ evolves as follows:

a) $D^{f(t)}=D^{f(0)}$,

b) For $z$ in $D^{f(0)}, V_{z}^{f(t)}=e^{-\mathbf{a} z^{j} t}\left(V_{z}^{f(0)}\right)$,

c) $v^{f(t)}(r)=e^{\mathbf{a} r^{j} t} v^{f(0)}(r) e^{-\mathbf{a} r^{j} t}$.

We next explain how to recover $f \in \mathcal{D}_{-}$from $S^{f}$. To do this first write $f=g h$ with $g \in \mathcal{D}_{-}^{\text {disc }}$ and $h \in \mathcal{D}_{-}^{\text {cont }}$. Then, $v^{f}=f_{-}^{-1} f_{+}=\left(g_{-} h_{-}\right)^{-1}\left(g_{+} h_{+}\right)=h_{-}^{-1} h^{+}$, since as we saw above, $g_{-}=g_{+}$. It follows from uniqueness of the Birkhoff decomposition that $v^{f}$ determines $h_{-}$and $h_{+}$and hence $h$. (Recall that $h$ in $\mathbf{C}_{+}$(respectively $\mathbf{C}_{-}$) is the unique meromorphic extension of $h_{+}$(respectively $\left.h_{-}\right)$.) On the other hand, from the poles $z$ of $g$ and the residues $\pi_{z}^{f}$ of $g$ at these poles we can recover $g$ and hence $f=g h$.

There is again an explicit formula for "pure solitons", or "reflectionless potentials" (i.e., $u \in P_{0}$ such that $f^{u}$ is in $\mathcal{D}_{-}^{\text {disc }}$ ). We will content ourselves here with writing the formula for the 1 -solitons of NLS, i.e., a single simple pole, say at $z=r+i s$, with residue the projection of $\mathbf{C}^{2}$ onto the vector $\left(\sqrt{1-|b|^{2}}, b\right)$, where $b \in \mathbf{C}$ with $|b|<1$. Then the solution $q(x, t)$ of NLS is:

$$
\frac{4 s b \sqrt{1-|b|^{2}} e^{\left(-2 i r x+\left(r^{2}-s^{2}\right) t\right)}}{e^{-2(s x+2 r s t)}\left(1-|b|^{2}\right)+e^{2(s x+2 r s t)}|b|^{2}} .
$$

(For $n$-soliton formulas, see [FT] for the $\mathbf{s u}(2)$ case and [TU2] for the $\mathbf{s u}(n)$ case.)

Recall that we have a natural bijection: $P_{0} \rightarrow \mathcal{D}_{-} \rightarrow \mathcal{D}_{-} / \mathcal{H}_{-}$, where the first arrow is the Scattering Transform, $\mathcal{F}_{\text {scat }}$, and the second is the natural coset projection. Since we have a natural action of $\mathcal{D}_{-}$on its coset space $\mathcal{D}_{-} / \mathcal{H}_{-}$, this induces an action of $\mathcal{D}_{-}$on $P_{0}$, and so the subgroups $\mathcal{D}_{-}^{\text {cont }}$ and $\mathcal{D}_{-}^{\text {disc }}$ also act on $P_{0}$. The 
orbit of 0 under $\mathcal{D}_{-}^{\text {disc }}$ give the reflectionless potentials or pure solitons, while the orbit of 0 under $\mathcal{D}_{-}^{\text {cont }}$ gives the potentials without poles.

We can now at last explain how the notion of Bäcklund transformation fits into this picture; namely the action of the generators $g_{z, \pi}$ of $\mathcal{D}_{-}^{\text {disc }}$ on $P_{0}$ are just the classical Bäcklund Transformations. Typically they add one to the number of solitons in a solution.

\section{REFERENCES}

[AC] Ablowitz, M.J., Clarkson, P.A., Solitons, non-linear evolution equations and inverse scattering, Cambridge Univ. Press, 1991. MR 93g:35108

[AKNS1] Ablowitz, M.J., Kaup, D.J., Newell, A.C. and Segur, H., Method for solving the SineGordon equation, Phys. Rev. Lett. 30 (1973), 1262-1264. MR 53:9967

[AKNS2] Ablowitz, M.J., Kaup, D.J., Newell, A.C. and Segur, H., The inverse scattering transform-Fourier analysis for nonlinear problems, Stud. Appl. Math. 53 (1974), 249-315. MR 56:9108

[AbM] Abraham, R., Marsden, J.E., Foundations of Mechanics, Benjamin/Cummings, 1978. MR 81e: 58025

[Ad] Adler, M., On a trace functional for formal pseudo-differential operators and the symplectic structure of the Korteweg-de Vries equation, Invent. Math 50 (1979), 219-248. MR 80i:58026

[AdM] Adler, M., van Moerbeke, P., Completely integrable systems, Euclidean Lie algebras and curves, Adv. Math. 38 (1980), 267-317. MR 83m:58041

[Ar] Arnold, V.I., Mathematical Methods of Classical Mechanics, Springer-Verlag, 1978. MR $\mathbf{5 7 : 1 4 0 3 3}$

[AA] Arnold, V.I., Avez, A., Ergodic Problems of Classical Mechanics, W. A. Benjamin, Inc., New York, 1968. MR 38:1233

[Au] Audin, M., Spinning Tops, Cambridge Univ. Press, 1996. MR 97i:58068

[BC1] Beals, R., Coifman, R.R., Scattering and inverse scattering for first order systems, Commun. Pure Appl. Math. 37 (1984), 39-90. MR 85f:34020

[BC2] Beals, R., Coifman, R.R., Inverse scattering and evolution equations, Commun. Pure Appl. Math. 38 (1985), 29-42. MR 86f:35153

[BC3] Beals, R., Coifman, R.R., Linear spectral problems, non-linear equations and the $\bar{\partial}$ method, Inverse Problems 5 (1989), 87-130. MR 90f:35171

[BS] Beals, R., Sattinger. D.H., On the complete integrability of complete integrable systems, Commun. Math. Phys. 138 (1991), 409-436. MR 92m:58051

[BDZ] Beals, R., Deift, P., Zhou, X., The inverse scattering transform on the line, in Important Developments in Soliton Theory, Springer, Berlin, 1993, pp. 7-32. MR 95k:34020

[Bi] Birkhoff, G.D., Proof of the Ergodic Theorem, Proc. Nat. Acad. Sci. USA 17 (1931), 650-660.

[BS] Bona, J.L. and Smith, R., The Initial-Value Problem for the Korteveg-de Vries Equation, Philos. Trans. Royal Soc. London, Series A 278 (1975), 555-601. MR 52:6219

[Bu] Budagov, A.S., A completely integrable model of classical field theory with nontrivial particle interaction in two-dimensional space-time. Questions in quantum field theory and statistical physocs, Zap. Nauchn. Sem. Leningrad. Otdel. Mat. Inst. Steklov (LOMI) 77 (1978), 24-56, 229 (in Russian). MR 80e:81053

[BuC] Bullough, R.K., Caudrey, P.J., Solitons, Topics in Current Physics, vol. 117, SpringerVerlag, 1980. MR 82m:35001

[Da] Darboux, G., Leçons sur la théorie générale des surfaces, Chelsea, 1972. MR 53:81

[DaR] Da Rios, Rend. Circ. Mat. Palermo 22 (1906), 117-135.

[DJ] Drazin, P.G., Johnson, R.S., Solitons: an introduction, Cambridge Univ. Press, 1989. MR 90j:35166

[Dr] Drinfel'd, V.G., Hamiltonian structures on Lie groups, Lie bialgebras and the geometric meaning of classical Yang-Baxter equations, Dokl. Akad. Nauk SSSR 268 (1983), 285287; Trans. as Sov. Math. Doklady 27 (1983), 68-71. MR 84i:58044 
[DS] Drinfel'd, V.G., and Sokolov, V.V., Equations of Korteweg-de Vries type and simple Lie algebras, Dokl. Akad. Nauk SSSR 258 (1981), 11-16; Trans. as Soviet Math. Dokl. 23, 457-462. MR 83k:58040

[Fe] Fermi, E., Beweis dass ein mechanisches Normalsysteme im Allgemeinen quasiergodisch ist, Phys, Zeit. 24 (1923), 261-265.

[FT] Faddeev, L.D., Takhtajan, L.A., Hamiltonian Methods in the Theory of Solitons, Springer-Verlag, 1987. MR 89m:58103

[FPU] Fermi, E., Pasta, J., Ulam, S., Studies of Nonlinear Problems. I, in Nonlinear Wave Motion, Lectures in Applied Math., vol. 15, Amer. Math. Soc., 1974, pp. 143-155. MR 49:790

[FNR1] Flaschka, H., Newell, A.C., Ratiu, T., Kac-Moody Lie algebras and soliton equations, II. Lax equations associated with $A_{1}^{(1)}$, Physica 9D (1983), 303-323. MR 86m:58067

[FNR2] Flaschka, H., Newell, A.C., Ratiu, T., Kac-Moody Lie algebras and soliton equations, IV. Lax equations associated with $A_{1}^{(1)}$, Physica 9D (1983), 333-345.

[FRS] Frenkel, I.E., Reiman, A.G., Semenov-Tian-Shansky, M.A., Graded Lie algebras and completely integrable dynamical systems, Dokl. Akad. Nauk SSSR 247 (1979), 802-805; Trans. as Soviet Math. Dokl. 20 (1979), 811-814. MR 81c:58042

[G] Gardner, C.S., The Korteweg-de Vries Equation as a Hamiltonian system, J. Math. Physics 12 (1971), 1548-1551. MR 44:3615

[GGKM] Gardner, C.S., Greene, J.M., Kruskal, M.D., Miura, R.M., Method for solving the Korteweg-de Vries equation, Physics Rev. Lett. 19 (1967), 1095-1097.

[GDi] Gel'fand, I.M., Dikii, L.A., Fractional Powers of Operators and Hamiltonian Systems, Funkcional'nyi Analiz i ego Prilozhenija 10 (1976). MR 55:6484

[GDo] Gel'fand, I.M., Dorfman, I. Ya, Hamiltonian operators and algebraic structures associated with them, Functional Anal. Appl. 13 (1979), 13-30, 96. MR 81c:58035

[GL] Gel'fand, I.M., Levitan, B. M., On the determination of a differential equation from its spectral function, Izv. Akad. Nauk SSSR Ser. Mat. 15 (1951), 309-360. MR 13:558f

[Ha] Hasimoto, H., Motion of a vortex filament and its relation to elastic, J. Phys. Soc. Japan 31 (1971), 293-295.

[HaK] Hasimoto, H., Kodama, Y., Solitons in Optical Communications, Clarendon Press, Oxford, 1995

[Ka1] Kato, T., On the Cauchy Problem for the (Generalized) Korteweg-de Vries Equation, Studies in Applied Math., Adv. in Math. Supp. Stud. 8 (1983), 93-128. MR 86f:35160

[Ka2] Kato, T., Quasi-linear equations of evolution, with applications to partial differential equations, Lecture Notes in Math., vol. 448, Springer-Verlag, Berlin and New York, 1975, pp. 25-70. MR 53:11252

$[\mathrm{KdV}] \quad$ Korteweg, D.J., de Vries, G., On the change of form of long waves advancing in a rectangular canal, and on a new type of long stationary waves, Philos. Mag. Ser. 539 (1895), 422-443.

[Kos] Kostant, B., The solution to a generalized Toda lattice and representation theory, Adv. Math. 34 (1979), 195-338. MR 82f:58045

$[\mathrm{KM}] \quad$ Kay, B., Moses, H.E., The determination of the scattering potential from the spectral measure function, III, Nuovo Cim. 3 (1956), 276-304.

[KS] Klein, F., Sommerfeld A., Theorie des Kreisels, Teubner, Liepzig, 1897.

[L] Lamb, G.L., Jr., Elements of Soliton Theory, John Wiley \& Sons, New York, 1980. MR 82f:35165

[La1] Lax, P.D., Integrals of nonlinear equations of evolution and solitary waves, Comm. Pure. Appl. Math. 21 (1968), 467-490. MR 38:3620

[La2] Lax, P.D., Periodic Solutions of the KdV Equations, in Nonlinear Wave Motion, Lectures in Applied Math., vol. 15, Amer. Math. Soc., 1974, pp. 85-96. MR 49:9384

[La3] Lax, P.D., Outline of a theory of the KdV equation, in Recent Mathematical Methods in Nonlinear Wave Propogation, Lecture Notes in Math., vol. 1640, Springer-Verlag, Berlin and New York, 1996, pp. 70-102.

[LA] Luther, G.G., Alber, M.S., Nonlinear Waves, Nonlinear Optics, and Your Communications Future, in Nonlinear Science Today, Springer-Verlag New York, Inc., 1997.

[M] Marchenko,V.A., On the reconstruction of the potential energy from phases of the scattered waves, Dokl. Akad. Nauk SSSR 104 (1955), 695-698. 
[N] Newell, A.C., Solitons in Mathematics and Physics, SIAM, CBMS-NSF vol. 48, 1985. MR 87h:35314

[NMPZ] Novikov, S., Manakov, S., Pitaevskii, L.B., Zakharov, V.E., Theory of Solitons, Plenum, New York, 1984. MR 86k:35142

[OU] Oxtoby, J.C., Ulam, S.M., Measure Preserving Homeomorphisms and Metrical Transitivity, Annals of Math. 42 (1941), 874-920. MR 3:211b

[PT] Palais, R.S., and Terng, C.L., Critical Point Theory and Submanifold Geometry, Lecture Notes in Math., vol. 1353, Springer-Verlag, Berlin and New York, 1988. MR 90c:53143

[Pe] Perelomov, A.M., Integrable Systems of Classical Mechanics and Lie Algebras, Birkhäuser Verlag, Basel, 1990. MR 91g:58127

[PrS] Pressley, A. and Segal, G. B., Loop Groups, Oxford Science Publ., Clarendon Press, Oxford, 1986. MR 88i:22049

[RS] Reyman, A.G., Semenov-Tian-Shansky, M.A., Current algebras and non-linear partial differential equations, Sov. Math., Dokl. 21 (1980), 630-634.

[Ri] Rica, R.L., Rediscovery of the Da Rios Equation, Nature 352 (1991), 561-562.

$[\mathrm{Ru}] \quad$ Russell, J.S., Report on Waves, 14th Mtg. of the British Assoc. for the Advance. of Science, John Murray, London, pp. 311-390 + 57 plates, 1844.

[Sa] Sattinger, D.H., Hamiltonian hierarchies on semi-simple Lie algebras, Stud. Appl. Math. 72 (1985), 65-86. MR 86b:58063

[SW] Segal, G., Wilson, G., Loop groups and equations of KdV type, Publ. Math. IHES 61 (1985), 5-65. MR 87b:58039

[Se1] Semenov-Tian-Shansky, M.A., Dressing transformations and Poisson group actions, Publ. RIMS Kyoto Univ. 21 (1985), 1237-1260. MR 88b:58057

[Se2] Semenov-Tian-Shansky, M.A., Classical r-matrices, Lax equations, Poisson Lie groups, and dressing transformations, Lecture Notes in Physics, Springer-Verlag, vol. 280, 1987, pp. 174-214. MR 89g:58098

[Sh] Shabat, A.B., An inverse scattering problem, Diff. Uravneniya 15 (1979), 1824-1834; Trans. in Diff. Equ. 15 (1980), 1299-1307. MR 81m:34026

[St] Strang, G., On the Construction and Comparison of Difference Schemes, SIAM J. Numerical Analysis 5 (1968), 506-517. MR 38:4057

[Sy] Symes, W.W., Systems of Toda type, Inverse spectral problems, and representation theory, Inventiones Math. 59 (1980), 13-51. MR 81g:58019

[Ta] Tappert, F., Numerical Solutions of the Korteweg-de Vries Equations and its Generalizations by the Split-Step Fourier Method, in Nonlinear Wave Motion, Lectures in Applied Math., vol. 15, Amer. Math. Soc., 1974, pp. 215-216. MR 49:790

[Te1] Terng, C.L., A higher dimensional generalization of the Sine-Gordon equation and its soliton theory, Ann. Math. 111 (1980), 491-510. MR 82j:58069

[Te2] Terng, C.L., Soliton equations and differential geometry, J. Differential Geometry 45 (1997), 407-445. CMP 97:13

[TU1] Terng, C.L., Uhlenbeck, K., Poisson Actions and Scattering Theory for Integrable Systems, dg-ga/9707004 (to appear).

[TU2] Terng, C.L., Uhlenbeck, K., Bäcklund transformations and loop group actions (to appear).

[U1] Uhlenbeck, K., Harmonic maps into Lie group (classical solutions of the chiral model), J. Differential Geometry 30 (1989), 1-50. MR 90g:58028

[U2] Uhlenbeck, K., On the connection between harmonic maps and the self-dual Yang-Mills and the Sine-Gordon equations, J. Geom. Phys. 8 (1992), 283-316. MR 93f:58050

[Ul] Ulam, S. M., Adventures of a Mathematician, Univ. of Calif. Press, 1991. MR 58:4954

[Wa] Wadati, M., The modified Korteweg-de Vries equation, J. Phys. Soc. Japan 34 (1973), 1289-1296. MR 51:7472

[Wi] Wilson, G., The modified Lax equations and two dimensional Toda lattice equations associated with simple Lie algebras, Ergodic Theory and Dynamical Systems I 30 (1981), 361-380. MR 84b:58058

[ZK] Zabusky, N.J., Kruskal, M.D., Interaction of solitons in a collisionless plasma and the recurrence of initial states, Physics Rev. Lett. 15 (1965), 240-243.

[ZF] Zakharov, V.E., Faddeev, L.D., Korteweg-de Vries equation is a fully integrable Hamiltonian system, Funktsional Anal. i Prilozhen 5 (1971), 18-27. MR 46:2270 
[ZMa1] Zakharov, V.E., Manakov, S.V., On resonant interaction of wave packets in non-linear media, JETP Letters 18 (1973), 243-247.

[ZMa2] Zakharov, V.E., Manakov, S.V., The theory of resonance interaction of wave packets in non-linear media, Sov. Phys. JETP 42 (1975), 842-850. MR 54:14617

[ZMi1] Zakharov, V.E., Mikhailov, A.V., Example of nontrivial interaction of solitons in twodimensional classical field theory, JETP Letters 27 (1978), 42-46.

[ZMi2] Zakharov, V.E., Mikhailov, A.V., Relativistically invariant two-dimensional models of field theory which are integrable by means of the inverse scattering problem method, Soviet Physics JETP 47 (1978), 1017-1027. MR 80c:81115

[ZS] Zakharov, V.E., Shabat, A.B., Exact theory of two-dimensional self-focusing and onedimensional self-modulation of waves in nonlinear media, Sov. Phys. JETP 34 (1972), 62-69. MR 53:9966

Department of Mathematics, Brandeis University, Waltham, Massachusetts 02254

Current address: The Institute for Advanced Study, Princeton, New Jersey 08540

E-mail address: palais@math.brandeis.edu 